\title{
The Second Generation Catalytic Asymmetric Synthesis of Tamiflu: Allylic Substitution Route
}

\author{
Tsuyoshi Mita, Nobuhisa Fukuda, Francesc X. Roca, \\ Motomu Kanai,* and Masakatsu Shibasaki* \\ Graduate School of Pharmaceutical Sciences, The University of Tokyo, 7-3-1 Hongo, \\ Bunkyo-ku, Tokyo 113-0033, Japan \\ mshibasa@mol.f.u-tokyo.ac.jp
}

Supporting Information 


\section{Table of Contents}

General

(A) Catalytic Enantioselective Ring-Opening Reaction of Aziridine with $\mathrm{TMSN}_{3}$ on $30 \mathrm{~g}$-Scale

(B) Asymmetric Synthesis of Tamiflu ${ }^{\circledR}$-Preliminary Optimization of the Allylic Substitution Route

(C) Asymmetric Synthesis of Tamiflu ${ }^{\circledR}$-Optimized Allylic Substitution Route

(D) Estimation of the Allylic Phosphate (28) Structure

(E) Comparison of ${ }^{1} \mathrm{H}$ NMR Spectra of Tamiflu-Free (37)

S18

(F) ${ }^{1} \mathrm{H}$ NMR and ${ }^{13} \mathrm{C}$ NMR Spectral Data for Compounds

S19 
General: Infrared (IR) spectra were recorded on a JASCO FT/IR 410 Fourier transform infrared spectrophotometer. NMR spectra were recorded on a JEOL JNM-LA500 spectrometer, operating at 500 $\mathrm{MHz}$ for ${ }^{1} \mathrm{H} \mathrm{NMR}, 126.65 \mathrm{MHz}$ for ${ }^{13} \mathrm{C} \mathrm{NMR}$. Chemical shifts in $\mathrm{CDCl}_{3}$ were reported in the scale relative to $\mathrm{CHCl}_{3}(7.26 \mathrm{ppm})$ for ${ }^{1} \mathrm{H} \mathrm{NMR}$, and to $\mathrm{CDCl}_{3}(77.0 \mathrm{ppm})$ for ${ }^{13} \mathrm{C} \mathrm{NMR}$, as internal references. Optical rotations were measured on a JASCO P-1010 polarimeter. ESI mass spectra were measured on Water-ZQ4000. EI Mass spectra were measured on JEOL JMS-BU20 GCmate. FAB mass spectra were measured on JEOL MStation JMS-700. Column chromatographies were performed with silica gel Merck 60 (230-400 mesh ASTM). The enantiomeric excesses (ee's) were determined by HPLC. HPLC analysis was performed on JASCO HPLC systems containing of following: pump, PU-980; detector, UV-970, measured at $254 \mathrm{~nm}$; column, Daicel Chiralpak AD-H or Daicel Chiralcel OD-H; mobile phase, 2-propanol/hexane; flow rate, $1.0 \mathrm{~mL} / \mathrm{min}$. In general, reactions were carried out in dry solvents under an argon atmosphere, unless noted otherwise. Dry solvents of tetrahydrofuran (THF) and dichloromethane $\left(\mathrm{CH}_{2} \mathrm{Cl}_{2}\right)$ were purchased from Kanto Chemical. Co., Inc. Propionitrile was distilled from calcium hydride. Other reagents were purified by usual methods. $\mathrm{Y}\left(\mathrm{O}^{i} \mathrm{Pr}\right)_{3}$ was purchased from Kojundo Chemical Laboratory Co., Ltd. (Fax: +81-492-84-1351, sales@kojundo.co.jp). Chiral ligand 2 was prepared by a reported method. ${ }^{1}$ This ligand is commercially available from Junsei Chemical. Co., Ltd. (Fax: +81-3-3270-5461) Caution! Azide compounds are potentially explosive, especially when concentrated to dryness.

\section{(A) Catalytic Enantioselective Ring-Opening Reaction of Aziridine with $\mathrm{TMSN}_{3}$ on 30 g-Scale ${ }^{2}$}

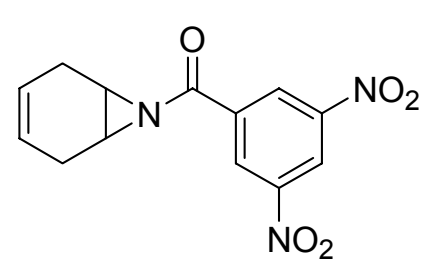

$328.9 \mathrm{~g}$
$\mathrm{Y}\left(\mathrm{O}^{\mathrm{i}} \mathrm{Pr}\right)_{3}(1 \mathrm{~mol} \%)$

$2(2 \mathrm{~mol} \%)$

$\mathrm{TMSN}_{3}$ (1.5 eq.)

2,6-dimethylphenol (1 eq.)

$\mathrm{CH}_{3} \mathrm{CH}_{2} \mathrm{CN}$, r.t., $12 \mathrm{~h}$

$94 \%, 89 \%$ ee

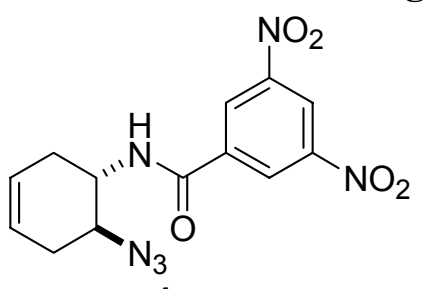

4

To a solution of ligand $2(0.92 \mathrm{~g}, 2.0 \mathrm{mmol}, 2 \mathrm{~mol} \%)$ in THF $(30 \mathrm{~mL}), \mathrm{Y}\left(\mathrm{O}^{i} \mathrm{Pr}\right)_{3}(0.2 \mathrm{M}$ in THF, $5 \mathrm{~mL}$, $1.0 \mathrm{mmol}, 1 \mathrm{~mol} \%$ ) was added at room temperature. The mixture was stirred at $45-60{ }^{\circ} \mathrm{C}$ for $1 \mathrm{~h}$, and then the solvent was evaporated. After drying the resulting pre-catalyst under reduced pressure $(<5 \mathrm{mmHg})$ for $2 \mathrm{~h}$, aziridine $3(28.9 \mathrm{~g}, 0.10 \mathrm{~mol})^{2}$, 2,6-dimethylphenol (12.2 g, $0.10 \mathrm{mmol}, 1$ equiv), and propionitrile $(500 \mathrm{~mL})$ were added at room temperature. After $10 \mathrm{~min}, \mathrm{TMSN}_{3}(19.6 \mathrm{~mL}, 0.15 \mathrm{~mol}, 1.5$ equiv) was added to start the reaction. After $12 \mathrm{~h}$, water was added followed by the addition of toluene. The organic layer was separated, and washed with $1 \mathrm{M} \mathrm{NaOH}$ several times. The solvent was removed under reduced pressure to afford 4 (31.5 g, 0.094 mmol, calculated by ${ }^{1} \mathrm{H}$ NMR analysis. $)^{2}$ in $94 \%$ yield as colorless solid. The enantiomeric excess of the product was determined by HPLC analysis to be $89 \%$ ee. Enantiomerically enriched $4(99 \%$ ee) was obtained after recrystallization from 2-propanol (recrystallization yield; $72 \%$ ).

1 Kato, N.; Tomita, D.; Maki, K.; Kanai, M.; Shibasaki, M. J. Org. Chem. 2004, 69, 6128.

2 Fukuta, Y.; Mita, T.; Fukuda, N.; Kanai, M.; Shibasaki, M. J. Am. Chem. Soc. 2006, 128, 6312. 
(B) Asymmetric Synthesis of Tamiflu ${ }^{\circledR}$-Preliminary Optimization of the Allylic Substitution Route

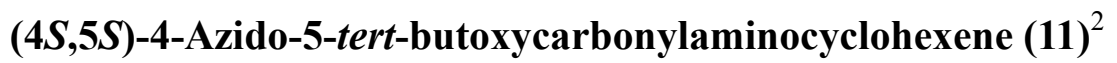

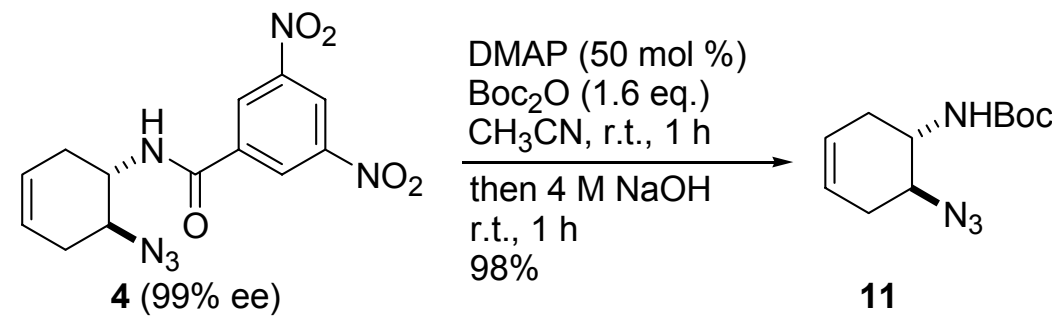

To a solution of 4 (11.36 g, $34.2 \mathrm{mmol}, 99 \%$ ee) in $\mathrm{CH}_{3} \mathrm{CN}(200 \mathrm{~mL}), \mathrm{Boc}_{2} \mathrm{O}$ (11.9 g, $54.5 \mathrm{mmol}, 1.6$ equiv) and DMAP (2.09 g, $17.1 \mathrm{mmol}, 50 \mathrm{~mol} \%$ ) were added, and the mixture was stirred at room temperature for 1 h. $4 \mathrm{M} \mathrm{NaOH}(150 \mathrm{~mL})$ was added and the mixture was stirred at room temperature for $1 \mathrm{~h}$. Water was added, and the product was extracted with AcOEt twice. The combined organic layer was dried over $\mathrm{Na}_{2} \mathrm{SO}_{4}$ and concentrated. The residue was purified by flash column chromatography (silica gel, hexane-AcOEt, 5:1) to afford $\mathbf{1 1}(7.96 \mathrm{~g}, 33.4 \mathrm{mmol})^{2}$ as a colorless solid in 98\% yield (2 steps).

(4S,5S)-4-Acetoamido-5-tert-butoxycarbonylaminocyclohexene (30)

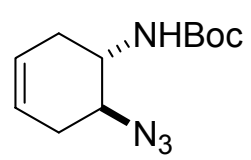

11

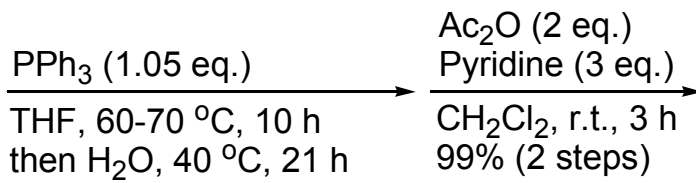

then $\mathrm{H}_{2} \mathrm{O}, 40^{\circ} \mathrm{C}, 21 \mathrm{~h}$

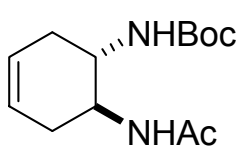

30

To a solution of $11(6.50 \mathrm{~g}, 27.3 \mathrm{mmol})$ in THF $(150 \mathrm{~mL}), \mathrm{PPh}_{3}(7.20 \mathrm{~g}, 27.3 \mathrm{mmol}, 1.0$ equiv) was added portion wise, and the mixture was stirred at $60-70{ }^{\circ} \mathrm{C}$. After $2.5 \mathrm{~h}$, additional $\mathrm{PPh}_{3}(0.36 \mathrm{~g}, 1.37$ mmol, 0.05 equiv) was added. After $7.5 \mathrm{~h}$, water $(20 \mathrm{~mL})$ was added, and the reaction mixture was stirred at $40{ }^{\circ} \mathrm{C}$ for $21 \mathrm{~h}$. After most of THF was removed under reduced pressure, water was removed by azeotropic evaporation with toluene (three times). To the residue, $\mathrm{CH}_{2} \mathrm{Cl}_{2}(80 \mathrm{~mL})$, pyridine $(6.6 \mathrm{~mL}, 81.8$ mmol, 3 equiv), and $\mathrm{Ac}_{2} \mathrm{O}(5.2 \mathrm{~mL}, 54.6 \mathrm{mmol}, 2$ equiv) were added, and the resulting mixture was stirred at room temperature. After $2 \mathrm{~h}$, water was added and the product was extracted with $\mathrm{CH}_{2} \mathrm{Cl}_{2}$ twice, and the combined organic layer was dried over $\mathrm{Na}_{2} \mathrm{SO}_{4}$. The solvent was removed under reduced pressure, and the residue was purified by column chromatography (silica gel, hexane-AcOEt, 4:1 to 0:1) to afford a mixture of $\mathrm{P}(\mathrm{O}) \mathrm{Ph}_{3}$ and 30, which was used for next step without further purification. The yield was calculated by ${ }^{1} \mathrm{H}$ NMR analysis (6.92 g, $27.2 \mathrm{mmol}$; 99\% yield (2 steps)). The analytically pure 30 was obtained through the purification by column chromatography (silica gel, hexane-acetone, $2: 1)$. IR (KBr): 3322, 2970, 1682, 1647, 1529, 1371, 1308, 1161, 1020, $661 \mathrm{~cm}^{-1} ;{ }^{1} \mathrm{H}$ NMR $\left(\mathrm{CDCl}_{3}\right): \delta=6.33(\mathrm{~d}, J=7.4$ $\mathrm{Hz}, 1 \mathrm{H}), 5.56(\mathrm{~s}, 2 \mathrm{H}), 4.76(\mathrm{~d}, J=8.5 \mathrm{~Hz}, 1 \mathrm{H}), 3.97-3.86(\mathrm{~m}, 1 \mathrm{H}), 3.74-3.62(\mathrm{~m}, 1 \mathrm{H}), 2.54-2.37(\mathrm{~m}, 2 \mathrm{H})$, 2.05-1.89 (m, 5H), $1.41(\mathrm{~s}, 9 \mathrm{H}) \mathrm{ppm} ;{ }^{13} \mathrm{C} \mathrm{NMR}\left(\mathrm{CDCl}_{3}\right): \delta=170.4,156.9,125.2,124.6,79.6,51.4,50.3$, 32.6, 32.4, 28.3, 23.3 ppm; MS (ESI): $m / z 277\left[\mathrm{M}+\mathrm{Na}^{+}\right]$; HRMS (EI): $m / z$ calcd for $\mathrm{C}_{13} \mathrm{H}_{22} \mathrm{~N}_{2} \mathrm{O}_{3}\left[\mathrm{M}^{+}\right]$: 254.1625. Found: 254.1614; $[\alpha]^{26}{ }_{\mathrm{D}}-73.7\left(c=1.410, \mathrm{CHCl}_{3}\right)$. 


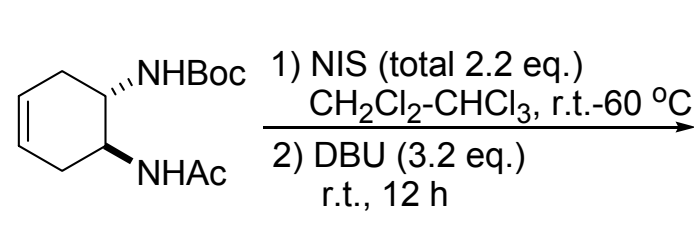

30

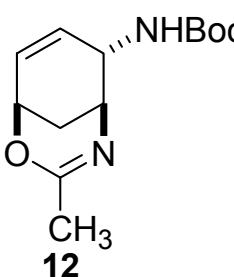

12

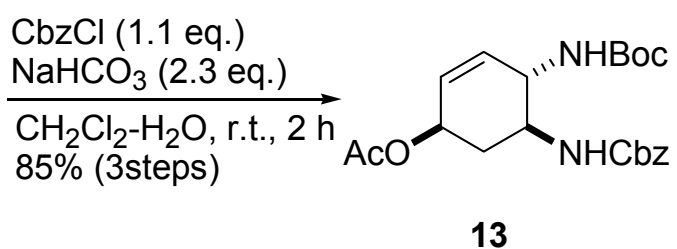

13

To a solution of $30(804 \mathrm{mg}, 3.16 \mathrm{mmol})$ containing $\mathrm{P}(\mathrm{O}) \mathrm{Ph}_{3}$ in $\mathrm{CH}_{2} \mathrm{Cl}_{2}(50 \mathrm{~mL})$, NIS (1.08 g, 4.80 mmol, 1,5 equiv) was added, and the resulting mixture was stirred at room temperature for $72 \mathrm{~h}$. Additional NIS ( $0.5 \mathrm{~g}, 2.22 \mathrm{mmol}, 0.7$ equiv) in $\mathrm{CHCl}_{3}(25 \mathrm{~mL})$ was added, and the resulting mixture was stirred at $40-60{ }^{\circ} \mathrm{C}$ for $10 \mathrm{~h}$. After 30 was consumed, 1,8-diazabicyclo[5.4.0]undec-7-ene (DBU: $1.5 \mathrm{~mL}$, $10.0 \mathrm{mmol}, 3.2$ equiv) was added in one portion at room temperature. After $12 \mathrm{~h}$, the solvent was removed under reduced pressure, and the residue was purified by column chromatography roughly (silica gel, hexane-AcOEt, 1:1 to $0: 1)$ to afford a mixture of $\mathrm{P}(\mathrm{O}) \mathrm{Ph}_{3}(12.9 \mathrm{mg}, 0.036 \mathrm{mmol})$, succinimide and 12, which was used for next step without further purification. Analytical date of 12: IR (KBr): 3201, 2967, $1705,1657,1530,1250,1176,1052,1021,816 \mathrm{~cm}^{-1} ;{ }^{1} \mathrm{H}$ NMR $\left(\mathrm{CDCl}_{3}\right): \delta=6.00(\mathrm{dd}, J=5.7,9.4 \mathrm{~Hz}, 1 \mathrm{H})$, $5.90(\mathrm{dd}, J=4.6,9.4 \mathrm{~Hz}, 1 \mathrm{H}), 4.63-4.49$ (m, 2H), 4.22 (brs, 1H), 3.74 (brs, $1 \mathrm{H}), 1.90-1.76$ (m, 5H), 1.43 (s, 9H) ppm; ${ }^{13} \mathrm{C} \mathrm{NMR}\left(\mathrm{CDCl}_{3}\right): \delta=156.6,154.5,130.1,127.3,79.7,67.0 / 65.2$ (rotamer), 50.0, 49.1, 28.33/28.28 (rotamer), 22.8, 21.8 ppm; MS (ESI): $m / z 253\left[\mathrm{M}+\mathrm{H}^{+}\right]$and $275\left[\mathrm{M}+\mathrm{Na}^{+}\right]$; HRMS (EI): $m / z$ calcd for $\mathrm{C}_{13} \mathrm{H}_{20} \mathrm{~N}_{2} \mathrm{O}_{3}\left[\mathrm{M}^{+}\right]$: 252.1468. Found: 252.1455. $[\alpha]^{22}+183.1\left(c=1.230, \mathrm{CHCl}_{3}\right)$.

To a solution of $\mathrm{NaHCO}_{3}\left(598 \mathrm{mg}, 7.12 \mathrm{mmol}, 2.3\right.$ equiv) and 12 in $\mathrm{CH}_{2} \mathrm{Cl}_{2}-\mathrm{H}_{2} \mathrm{O}(20-10 \mathrm{~mL}), \mathrm{CbzCl}$ (500 $\mu \mathrm{L}, 3.50 \mathrm{mmol}, 1.1$ equiv) was added dropwise. After $2 \mathrm{~h}$, water was added and the product was extracted with $\mathrm{CH}_{2} \mathrm{Cl}_{2}$ twice, and the combined organic layer was dried over $\mathrm{Na}_{2} \mathrm{SO}_{4}$. The solvent was removed under reduced pressure, and the residue was purified by column chromatography (silica gel, hexane-AcOEt, 4:1 to 2:1) to afford pure $13(1.09 \mathrm{~g}, 2.69 \mathrm{mmol})$ as a colorless solid in $85 \%$ yield (3 steps). In this purification, $\mathrm{P}(\mathrm{O}) \mathrm{Ph}_{3}$ and succinimide could be removed completely. IR (KBr): 3336, 2968, 1733, 1675, 1520, 1300, 1239, 1171, 1024, $636 \mathrm{~cm}^{-1} ;{ }^{1} \mathrm{H}$ NMR $\left(\mathrm{CDCl}_{3}\right): \delta=7.33$ (brs, 5H), $5.70(\mathrm{~d}, J=$ $10.1 \mathrm{~Hz}, 1 \mathrm{H}), 5.68(\mathrm{~d}, J=10.1 \mathrm{~Hz}, 1 \mathrm{H}), 5.46$ (brs, 1H), 5.39 (brs, 1H), $5.08(\mathrm{~s}, 2 \mathrm{H}), 4.81-4.67$ (m, 1H), 4.29-4.17 (m, 1H), 3.74-3.59 (m, 1H), 2.53-3.37 (m, 1H), $2.04(\mathrm{~s}, 3 \mathrm{H}), 1.73-1.60(\mathrm{~m}, 1 \mathrm{H}), 1.41(\mathrm{~s}, 9 \mathrm{H})$ ppm; ${ }^{13} \mathrm{C} \mathrm{NMR}\left(\mathrm{CDCl}_{3}\right): \delta=170.3,156.4,156.2,136.4,130.6,129.3,128.5,128.04,127.96,80.1,68.7$, 66.7, 52.7, 51.8, 34.6, 28.2, $21.1 \mathrm{ppm}$; MS (ESI): $m / z 427\left[\mathrm{M}+\mathrm{Na}^{+}\right]$; HRMS (EI): $m / z$ calcd for $\mathrm{C}_{21} \mathrm{H}_{28} \mathrm{~N}_{2} \mathrm{O}_{6}\left[\mathrm{M}^{+}\right]$: 404.1942. Found: 404.1943; $[\alpha]^{26}{ }_{\mathrm{D}}+99.4\left(c=1.710, \mathrm{CHCl}_{3}\right)$.

$(3 R, 5 S, 6 S)-5$-Benzyloxycarbonylamino-6-tert-butoxycarbonylamino-3-hydroxycyclohexene (31)

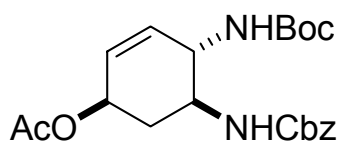

13

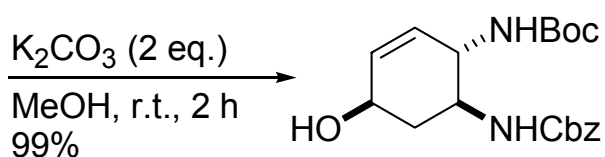

31

To a solution of 13 (6.27 g, $15.5 \mathrm{mmol})$ in $\mathrm{MeOH}(400 \mathrm{~mL}), \mathrm{K}_{2} \mathrm{CO}_{3}$ (4.30 g, $31.0 \mathrm{mmol}$, 2 equiv) was added and the mixture was stirred at room temperature for $2 \mathrm{~h}$. After most of $\mathrm{MeOH}$ was removed under reduced pressure, AcOEt and water were added, and the product was extracted with AcOEt twice. The 
organic layer was washed with brine, dried over $\mathrm{Na}_{2} \mathrm{SO}_{4}$, and concentrated to afford 31 (5.57 g, 15.4 mmol) as a colorless solid in 99\% yield. IR (KBr): 3344. 2951, 1675, 1525, 1276, 1063, 1025, 994, 700, $623 \mathrm{~cm}^{-1} ;{ }^{1} \mathrm{H}$ NMR $\left(d_{4}-\mathrm{MeOH}\right): \delta=7.41-7.22(\mathrm{~m}, 5 \mathrm{H}), 5.71(\mathrm{dd}, J=1.7,10.2 \mathrm{~Hz}, 1 \mathrm{H}), 5.50(\mathrm{~d}, J=10.2$ $\mathrm{Hz}, 1 \mathrm{H}), 5.08(\mathrm{~d}, J=12.5 \mathrm{~Hz}, 1 \mathrm{H}), 5.04(\mathrm{~d}, J=12.5 \mathrm{~Hz}, 1 \mathrm{H}), 4.33$ (brs, $1 \mathrm{H}), 4.09-4.02$ (m, 1H), 3.61-3.52 (m, 1H), 2.25-2.16 (m, 1H), 1.67-1.53 (m, 1H), $1.40(\mathrm{~s}, 9 \mathrm{H}) \mathrm{ppm} ;{ }^{13} \mathrm{C}$ NMR $\left(d_{4}-\mathrm{MeOH}\right): \delta=$ 158.55, 158.52, 138.3, 134.0, 130.0, 129.4, 128.9, 128.8, 80.3, 67.5, 67.4, 53.4, 53.1, 39.8, 28.7 ppm; MS (ESI): $m / z 385\left[\mathrm{M}+\mathrm{Na}^{+}\right]$; HRMS (EI): $m / z$ calcd for $\mathrm{C}_{19} \mathrm{H}_{27} \mathrm{~N}_{2} \mathrm{O}_{5}\left[\mathrm{M}+\mathrm{H}^{+}\right]: 363.1914$. Found: 363.1912; $[\alpha]_{\mathrm{D}}^{25}+90.7\left(c=0.635, \mathrm{CHCl}_{3}\right)$.

(5S,6S)-5-Benzyloxycarbonylamino-6-tert-butoxycarbonylaminocyclohexen-3-one (14)

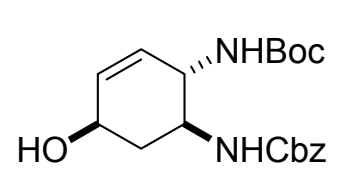

31

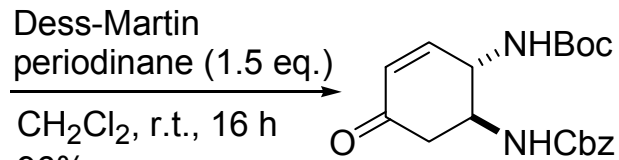

$14(99 \%$ ee $)$

To a solution of $31(5.57 \mathrm{~g}, 15.4 \mathrm{mmol})$ in $\mathrm{CH}_{2} \mathrm{Cl}_{2}(400 \mathrm{~mL})$, Dess-Martin periodinane $(9.9 \mathrm{~g}, 23.3$ mmol, 1.5 equiv) was added, and the mixture was stirred at room temperature. After $16 \mathrm{~h}$, saturated aqueous $\mathrm{Na}_{2} \mathrm{~S}_{2} \mathrm{O}_{3}$ was added and the organic layer was separated. The aqueous layer was extracted with $\mathrm{CH}_{2} \mathrm{Cl}_{2}$ twice, and the combined organic layer was washed with saturated aqueous $\mathrm{NaHCO}_{3}$ once and dried over $\mathrm{Na}_{2} \mathrm{SO}_{4}$. The solvent was removed under reduced pressure, and the residue was purified by column chromatography (silica gel, hexane-AcOEt, 4:1 to 2:1) to afford 14 (5.33 g, $14.8 \mathrm{mmol}$ ) as a colorless solid in $96 \%$ yield. Enantiomeric excess was determined by chiral HPLC to be $99 \%$. IR (KBr): 3289, 2979, 1688, 1552, 1284, 1251, 1168, 1045, 757, $697 \mathrm{~cm}^{-1} ;{ }^{1} \mathrm{H}$ NMR $\left(\mathrm{CDCl}_{3}\right)$ : $\delta=7.39-7.27(\mathrm{~m}$, $5 \mathrm{H}), 6.77(\mathrm{dd}, J=1.1,10.0 \mathrm{~Hz}, 1 \mathrm{H}), 6.04(\mathrm{~d}, J=10.0 \mathrm{~Hz}, 1 \mathrm{H}), 5.50(\mathrm{~d}, J=8.5 \mathrm{~Hz}, 1 \mathrm{H}), 5.16(\mathrm{~d}, J=8.5$ $\mathrm{Hz}, 1 \mathrm{H}), 5.09$ (d, $J=12.5 \mathrm{~Hz}, 1 \mathrm{H}), 5.06(\mathrm{~d}, J=12.5 \mathrm{~Hz}, 1 \mathrm{H}), 4.55-4.42(\mathrm{~m}, 1 \mathrm{H}), 4.09-3.96(\mathrm{~m}, 1 \mathrm{H}), 2.83$ $(\mathrm{dd}, J=4.0,16.4 \mathrm{~Hz}, 1 \mathrm{H}), 2.43(\mathrm{dd}, J=13.4,16.4 \mathrm{~Hz}, 1 \mathrm{H}), 1.41(\mathrm{~s}, 9 \mathrm{H}) \mathrm{ppm} ;{ }^{13} \mathrm{C} \mathrm{NMR}\left(\mathrm{CDCl}_{3}\right): \delta=$ $196.3,156.2$, 150.1, 141.8, 136.1, 130.3, 128.5, 128.2, 128.0, 80.5, 66.9, 53.1, 52.9, 43.8, 28.2 ppm; MS (ESI): $m / z 383\left[\mathrm{M}+\mathrm{Na}^{+}\right]$; HRMS (EI): $m / z$ calcd for $\mathrm{C}_{19} \mathrm{H}_{24} \mathrm{~N}_{2} \mathrm{O}_{5}\left[\mathrm{M}^{+}\right]: 360.1680$. Found: $360.1671 ;[\alpha]^{24}$ +90.3 ( $c=0.905, \mathrm{CHCl}_{3}, 99 \%$ ee). HPLC (Chiralpak AD-H, 2-propanol/hexane 1/9, flow $1.0 \mathrm{~mL} / \mathrm{min}$, detection at $254 \mathrm{~nm}$.): $\mathrm{t}_{\mathrm{R}} 20.0 \mathrm{~min}$ (major) and $27.6 \mathrm{~min}$ (minor).

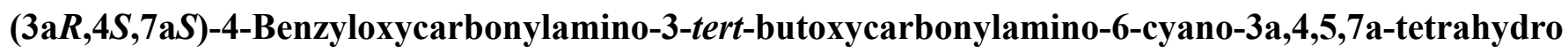
benzooxazol-2-one (16)

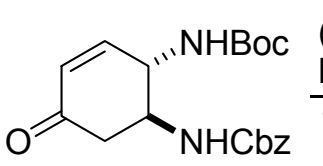

14<smiles>[R]C(=O)NC1CC(C#N)=C[C@@H]2OC(=O)N[C@@H]12</smiles>

32

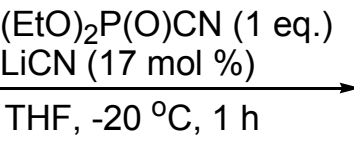

THF, $-20^{\circ} \mathrm{C}, 1 \mathrm{~h}$

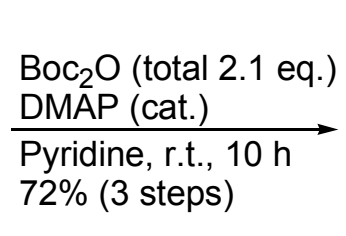

Pyridine, r.t., 10
$72 \%$ (3 steps)

16

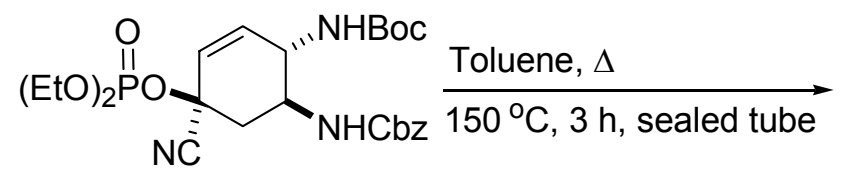

$15 d r=$ ca. $20: 1$

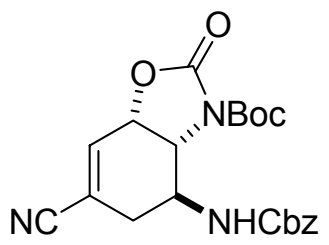

16<smiles>CC(=O)N(C)[C@@H]1CC(C#N)=C[C@@H]2OC(=O)N(C(=O)OCc3ccccc3)[C@H]21</smiles>

$33<5 \%$ Boc 
To a solution of 14 (148 mg, $0.411 \mathrm{mmol})$ in THF $(2 \mathrm{~mL})$, diethylphosphoryl cyanide $(64 \mu \mathrm{L}, 0.42$ mmol, 1 equiv) was added at room temperature. After cooling to $-20{ }^{\circ} \mathrm{C}$, $\mathrm{LiCN}(2.5 \mathrm{mg}, 0.068 \mathrm{mmol}, 17$ mol \%) was added in one portion. After stirring at $-20{ }^{\circ} \mathrm{C}$ for $1 \mathrm{~h}$, THF was removed under reduced pressure. The residue was diluted in toluene $(3 \mathrm{~mL})$, and heated at $150{ }^{\circ} \mathrm{C}$ in a sealed tube. After $3 \mathrm{~h}$, THF was removed under reduced pressure, and the residue was diluted with pyridine $(1 \mathrm{~mL})$. After the addition of a catalytic amount of DMAP, $\mathrm{Boc}_{2} \mathrm{O}$ (total $190 \mathrm{mg}, 0.87 \mathrm{mmol}, 2.1$ equiv) was added in portionwise for $10 \mathrm{~h}$ until 32 was completely consumed. Pyridine was removed under reduced pressure and the residue was purified by column chromatography (silica gel, hexane-AcOEt, 4:1 to 2:1) to afford 16 (122 $\mathrm{mg}, 0.295 \mathrm{mmol}$ ) as a colorless amorphous in $72 \%$ yield. The diastereoselectivity of 15 was determined by ${ }^{1} \mathrm{H}$ NMR analysis to be ca. 20:1. The configuration of the tetrasubstituted carbon of 15 was temporarily assigned. IR (neat): 3353, 2982, 2936, 2224, 1808, 1731, 1538, 1359, 1023, $757 \mathrm{~cm}^{-1} ;{ }^{1} \mathrm{H}$ $\operatorname{NMR}\left(\mathrm{CDCl}_{3}\right): \delta=7.40-7.29(\mathrm{~m}, 5 \mathrm{H}), 6.62(\mathrm{t}, J=3.3 \mathrm{~Hz}, 1 \mathrm{H}), 5.82(\mathrm{~d}, J=4.9 \mathrm{~Hz}, 1 \mathrm{H}), 5.12(\mathrm{~d}, J=12.5$ $\mathrm{Hz}, 1 \mathrm{H}), 5.09$ (d, $J=12.5 \mathrm{~Hz}, 1 \mathrm{H}), 5.05-4.99$ (m, 1H), 4.42 (dd, $J=7.4,10.4 \mathrm{~Hz}, 1 \mathrm{H}), 3.94-3.85(\mathrm{~m}, 1 \mathrm{H})$, $3.00(\mathrm{dd}, J=3.9,17.4 \mathrm{~Hz}, 1 \mathrm{H}), 2.34-2.23(\mathrm{~m}, 1 \mathrm{H}), 1.53(\mathrm{~s}, 9 \mathrm{H}) \mathrm{ppm} ;{ }^{13} \mathrm{C} \mathrm{NMR}\left(\mathrm{CDCl}_{3}\right): \delta=155.8,150.5$, 149.5, 135.9, 134.9, 128.5, 128.3, 128.1, 117.6, 116.4, 85.8, 69.5, 67.0, 55.5, 49.5, 31.2, 27.7 ppm; MS (ESI): $m / z 436\left[\mathrm{M}+\mathrm{Na}^{+}\right]$and 336 [M-Boc+H+Na ${ }^{+}$; HRMS (EI): $m / z$ calcd for $\mathrm{C}_{21} \mathrm{H}_{23} \mathrm{~N}_{3} \mathrm{O}_{6}\left[\mathrm{M}^{+}\right.$(minor)]: 413.1581. Found: 413.1574 and $m / z$ calcd for $\mathrm{C}_{16} \mathrm{H}_{15} \mathrm{~N}_{3} \mathrm{O}_{4}$ [M-Boc $+\mathrm{H}^{+}$(major)]: 313.1057. Found: $313.1060 ;[\alpha]^{25}+7.2\left(c=1.185, \mathrm{CHCl}_{3}\right)$.

$(3 S, 4 R, 5 S)-5$-Benzyloxycarbonylamino-4-tert-butoxycarbonylamino-1-cyano-3-hydroxycyclohexene (34)

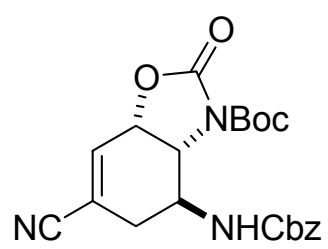

16

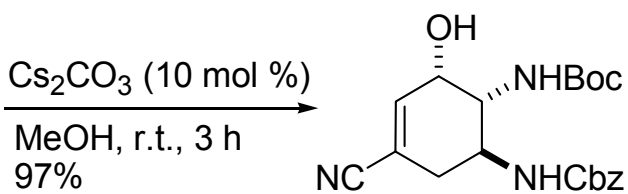

34

To a solution of 16 (1.36 g, $3.28 \mathrm{mmol})$ in $\mathrm{MeOH}(100 \mathrm{~mL}), \mathrm{Cs}_{2} \mathrm{CO}_{3}(107 \mathrm{mg}, 0.33 \mathrm{mmol}, 10 \mathrm{~mol} \%)$ was added and the mixture was stirred at room temperature. After $3 \mathrm{~h}, \mathrm{MeOH}$ was removed under reduced pressure, and the residue was purified by column chromatography (silica gel, hexane-AcOEt, 2:1) to afford 34 (1.24 g, $3.20 \mathrm{mmol}$ ) as a colorless solid in 97\% yield. IR (KBr): 3324, 2981, 2225, 1697, 1670, $1543,1281,1169,1043,741 \mathrm{~cm}^{-1} ;{ }^{1} \mathrm{H} \mathrm{NMR}\left(\mathrm{CDCl}_{3}\right): \delta=7.42-7.28(\mathrm{~m}, 5 \mathrm{H}), 6.55(\mathrm{~s}, 1 \mathrm{H}), 5.76(\mathrm{~d}, J=7.9$ $\mathrm{Hz}, 1 \mathrm{H}), 5.43(\mathrm{~d}, J=8.5 \mathrm{~Hz}, 1 \mathrm{H}), 5.07(\mathrm{~s}, 2 \mathrm{H}), 4.37-4.27(\mathrm{~m}, 1 \mathrm{H}), 4.05-3.94(\mathrm{~m}, 1 \mathrm{H}), 3.75-3.64(\mathrm{~m}, 1 \mathrm{H})$, $3.10(\mathrm{~d}, J=7.4 \mathrm{~Hz}, 1 \mathrm{H}), 2.82(\mathrm{dd}, J=5.1,16.5 \mathrm{~Hz}, 1 \mathrm{H}), 2.20(\mathrm{dd}, J=10.8,16.5 \mathrm{~Hz}, 1 \mathrm{H}), 1.41(\mathrm{~s}, 9 \mathrm{H})$ ppm; ${ }^{13} \mathrm{C} \mathrm{NMR}\left(\mathrm{CDCl}_{3}\right): \delta=157.1,156.8,141.6,136.0,128.5,128.2,127.8,117.4,114.1,80.4,67.0$, 65.3, 52.8, 47.4, 34.5, 28.2 ppm; MS (ESI): $m / z 410$ [M+Na ${ }^{+}$; HRMS (EI): $m / z$ calcd for $\mathrm{C}_{20} \mathrm{H}_{25} \mathrm{~N}_{3} \mathrm{O}_{5}$ $\left[\mathrm{M}^{+}\right]$: 387.1789. Found: 387.1787; $[\alpha]^{26}{ }_{\mathrm{D}}+65.1\left(c=0.625, \mathrm{CHCl}_{3}\right)$.

\footnotetext{
3 Yamagiwa, N.; Tian, J.; Matsunaga, S.; Shibasaki, M. J. Am. Chem. Soc, 2005, 127, 3413.
} 
(4R,5S)-5-Benzyloxycarbonylamino-4-tert-butoxycarbonylaminocyclohexen-3-one (35)

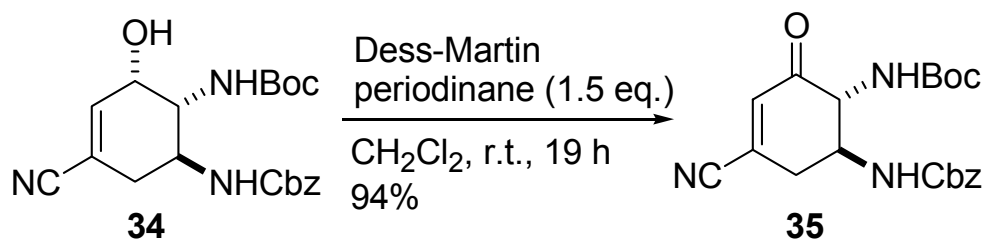

To a solution of $34(47.6 \mathrm{mg}, 0.123 \mathrm{mmol})$ in $\mathrm{CH}_{2} \mathrm{Cl}_{2}(5 \mathrm{~mL})$, Dess-Martin periodinane $(79.2 \mathrm{mg}, 0.19$ mmol, 1.5 equiv) was added and the mixture was stirred at room temperature. After $19 \mathrm{~h}, \mathrm{CH}_{2} \mathrm{Cl}_{2}$ was removed under reduced pressure, and the residue was purified through short pad column (silica gel, hexane-AcOEt, 2:1) to afford $35(44.6 \mathrm{mg}, 0.116 \mathrm{mmol}$ ) as a colorless solid in 94\% yield. The purification should be performed quickly because $\beta$-cyanoenone 35 was relatively unstable on a silica gel column chromatography. This reaction was also applicable on a 1.24 g scale. ( $80 \%$ yield). IR (KBr): 3311 , 2980, 2225, 1717, 1686, 1553, 1528, 1276, 1023, $758 \mathrm{~cm}^{-1} ;{ }^{1} \mathrm{H}$ NMR $\left(\mathrm{CDCl}_{3}\right): \delta=7.41-7.28(\mathrm{~m}, 5 \mathrm{H})$, $6.62(\mathrm{~d}, J=2.8 \mathrm{~Hz}, 1 \mathrm{H}), 6.34(\mathrm{~d}, J=6.8 \mathrm{~Hz}, 1 \mathrm{H}), 5.50(\mathrm{~d}, J=6.2 \mathrm{~Hz}, 1 \mathrm{H}), 5.09(\mathrm{~s}, 2 \mathrm{H}), 4.47-4.35(\mathrm{~m}$, $1 \mathrm{H}), 4.09-3.97(\mathrm{~m}, 1 \mathrm{H}), 3.12(\mathrm{dd}, J=4.5,18.1 \mathrm{~Hz}, 1 \mathrm{H}), 2.66(\mathrm{ddd}, J=2.8,10.5,18.1 \mathrm{~Hz}, 1 \mathrm{H}), 1.41$ (s, 9H) ppm; ${ }^{13} \mathrm{C} \mathrm{NMR}\left(\mathrm{CDCl}_{3}\right): \delta=192.2,157.4,156.1,137.1,136.1,129.5,128.5,128.1,127.9,115.8$, 81.3, 66.9, 60.1, 53.8, 35.4, 28.2, 28.0 ppm; MS (ESI): $m / z 408$ [M+Na $\left.{ }^{+}\right]$; HRMS (EI): $m / z$ calcd for $\mathrm{C}_{20} \mathrm{H}_{23} \mathrm{~N}_{3} \mathrm{O}_{5}\left[\mathrm{M}^{+}\right]$: 385.1632. Found: 385.1633; $[\alpha]^{26}{ }_{\mathrm{D}}-36.4\left(c=0.838, \mathrm{CHCl}_{3}\right)$.

(3R,4R,5S)-5-Benzyloxycarbonylamino-4-tert-butoxycarbonylamino-1-cyano-3-hydroxycyclohexene (17)<smiles>CC(=O)N[C@@H]1CC(C#N)=CC(=O)[C@@H]1NC(=O)OCc1ccccc1</smiles>

35

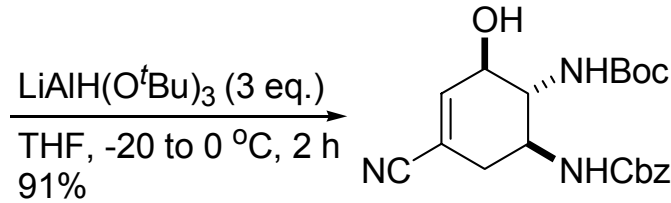

$17 \mathrm{dr}=>20: 1$

To a solution of $\mathrm{LiAlH}\left(\mathrm{O}^{t} \mathrm{Bu}\right)_{3}(1 \mathrm{M}$ in THF, $7.7 \mathrm{~mL}, 7.70 \mathrm{mmol}, 3$ equiv) in THF $(70 \mathrm{~mL}), 35$ (987 mg, $2.56 \mathrm{mmol})$ in THF $(30 \mathrm{~mL})$ was added, and the resulting mixture was stirred at $-20{ }^{\circ} \mathrm{C}$, and then warmed to $0{ }^{\circ} \mathrm{C}$. After $2 \mathrm{~h}$, saturated aqueous $\mathrm{NH}_{4} \mathrm{Cl}$ was added to quench the reaction. After removal of insoluble materials through a celite pad, the product was extracted with $\mathrm{CH}_{2} \mathrm{Cl}_{2}$ three times, and the combined organic layer was washed with brine and dried over $\mathrm{Na}_{2} \mathrm{SO}_{4}$. The solvent was removed under reduced pressure, and the residue was purified by column chromatography (silica gel, hexane-AcOEt, 4:1 to 1:1) to afford 17 (900 mg, $2.32 \mathrm{mmol}$ ) as a colorless solid in 91\% yield. The diastereoselectivity of the product was determined by ${ }^{1} \mathrm{H}$ NMR analysis to be $>20: 1$. IR (KBr): 3456, 3289, 2976, 2227, 1700, 1561, 1523, 1281, 1037, $994 \mathrm{~cm}^{-1} ;{ }^{1} \mathrm{H}$ NMR $\left(\mathrm{CDCl}_{3}\right): \delta=7.41-7.29(\mathrm{~m}, 5 \mathrm{H}), 6.49(\mathrm{~s}, 1 \mathrm{H}), 5.26(\mathrm{~d}, J=8.3 \mathrm{~Hz}, 1 \mathrm{H})$, $5.16(\mathrm{~d}, J=7.9 \mathrm{~Hz}, 1 \mathrm{H}), 5.15-5.06(\mathrm{~m}, 2 \mathrm{H}), 4.25$ (brs, 1H), 3.93-3.82 (m, 1H), 3.80 (brs, 1H), 3.64-3.53 $(\mathrm{m}, 1 \mathrm{H}), 2.70(\mathrm{dd}, J=4.3,17.4 \mathrm{~Hz}, 1 \mathrm{H}), 2.35-2.26(\mathrm{~m}, 1 \mathrm{H}), 1.41(\mathrm{~s}, 9 \mathrm{H}) \mathrm{ppm} ;{ }^{13} \mathrm{C} \mathrm{NMR}\left(\mathrm{CDCl}_{3}\right): \delta=$ 157.8, 156.7, 144.8, 135.9, 128.6, 128.3, 128.0, 117.2, 110.9, 80.9, 71.5, 67.2, 57.4, 49.1, 33.3, 28.2 ppm; MS (ESI): $m / z 410\left[\mathrm{M}+\mathrm{Na}^{+}\right]$; HRMS (EI): $m / z$ calcd for $\mathrm{C}_{20} \mathrm{H}_{25} \mathrm{~N}_{3} \mathrm{O}_{5}\left[\mathrm{M}^{+}\right]$: 387.1789. Found: 387.1788; $[\alpha]^{24}-63.0(c=0.825, \mathrm{MeOH})$. 
(3S,4R,5S)-5-Benzyloxycarbonylamino-3,4-tert-butoxycarbonylimino-1-cyanocyclohexene (18)

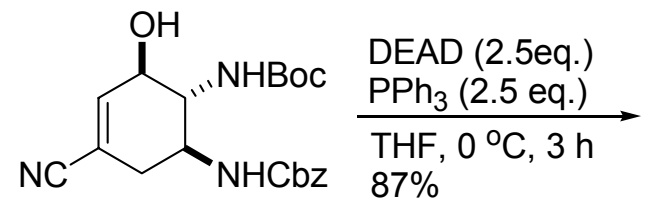

17

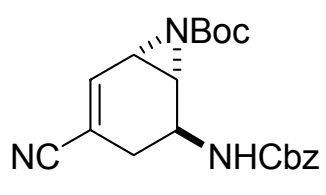

18

To a solution of $\mathrm{PPh}_{3}(78.7 \mathrm{mg}, 0.30 \mathrm{mmol}, 2.5$ equiv) in THF ( $2 \mathrm{~mL}), \mathrm{DEAD}(40 \%$ in toluene, $130 \mu \mathrm{L}$, $0.30 \mathrm{mmol}, 2.5$ equiv) and $17(45.9 \mathrm{mg}, 0.118 \mathrm{mmol})$ in $\mathrm{THF}(1 \mathrm{~mL})$ were added and the resulting mixture was stirred at $0{ }^{\circ} \mathrm{C}$. After $3 \mathrm{~h}$, mixture was concentrated, and purified by column chromatography (silica gel, hexane-AcOEt, 4:1 to $3: 1)$ to afford $18(37.9 \mathrm{mg}, 0.103 \mathrm{mmol}$ ) as a colorless amorphous in $87 \%$ yield. IR (neat): 3330, 2979, 2219, 1724, 1531, 1369, 1236, 1152, 1041, $752 \mathrm{~cm}^{-1}$; ${ }^{1} \mathrm{H}$ NMR $\left(\mathrm{CDCl}_{3}\right): \delta=7.44-7.29(\mathrm{~m}, 5 \mathrm{H}), 6.88(\mathrm{t}, J=3.7 \mathrm{~Hz}, 1 \mathrm{H}), 5.13(\mathrm{~d}, J=11.9 \mathrm{~Hz}, 1 \mathrm{H}), 5.08(\mathrm{~d}, J=11.9 \mathrm{~Hz}$, $1 \mathrm{H}), 4.78(\mathrm{~d}, J=8.5 \mathrm{~Hz}, 1 \mathrm{H}), 4.62$ (brs, $1 \mathrm{H}), 3.10$ (brs, 1H), 2.96 (t, $J=5.1 \mathrm{~Hz}, 1 \mathrm{H}), 2.58$ (d, $J=16.7 \mathrm{~Hz}$, $1 \mathrm{H}), 2.36(\mathrm{~d}, J=16.7 \mathrm{~Hz}, 1 \mathrm{H}), 1.45(\mathrm{~s}, 9 \mathrm{H}) \mathrm{ppm} ;{ }^{13} \mathrm{C} \mathrm{NMR}\left(\mathrm{CDCl}_{3}\right): \delta=159.9,155.4,140.3,135.8$, 128.6, 128.4, 128.3, 118.1, 112.3, 82.8, 67.3, 41.8, 41.3, 31.9, 30.0, 27.8 ppm; MS (ESI): $m / z 392$ $\left[\mathrm{M}+\mathrm{Na}^{+}\right]$; HRMS (EI): $m / z$ calcd for $\mathrm{C}_{20} \mathrm{H}_{23} \mathrm{~N}_{3} \mathrm{O}_{4}\left[\mathrm{M}^{+}\right]: 369.1683$. Found: 369.1628; $[\alpha]^{24}-51.9(c=$ $\left.0.750, \mathrm{CHCl}_{3}\right)$.

$(3 R, 4 R, 5 S)-5$-Benzyloxycarbonylamino-4-tert-butoxycarbonylamino-1-cyano-3-(1-ethylpropoxy)cycl ohexene (36)

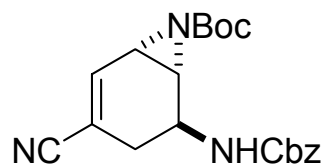

18

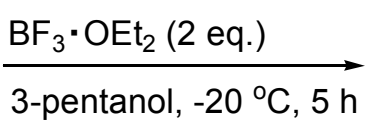

$$
\mathrm{NC}
$$

$3651 \%$

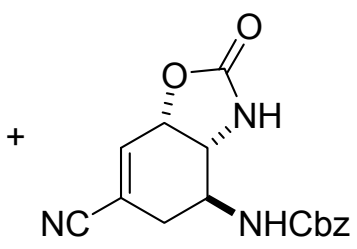

$3228 \%$

To a solution of $18(300 \mathrm{mg}, 0.812 \mathrm{mmol})$ in 3-pentanol $(8 \mathrm{~mL}), \mathrm{BF}_{3} \cdot \mathrm{OEt}_{2}(0.5 \mathrm{M}$ in 3-pentanol, 2.4 $\mathrm{mL}, 1.20 \mathrm{mmol}, 1.5$ equiv) was added dropwise, and the resulting mixture was stirred at $-20{ }^{\circ} \mathrm{C}$. After $3 \mathrm{~h}$, additional $\mathrm{BF}_{3} \cdot \mathrm{OEt}_{2}\left(0.5 \mathrm{M}\right.$ in 3-pentanol, $0.8 \mathrm{~mL}, 0.40 \mathrm{mmol}, 0.5$ equiv) was added dropwise at $-20{ }^{\circ} \mathrm{C}$. After $2 \mathrm{~h}$, saturated aqueous $\mathrm{NaHCO}_{3}$ was added to quench the reaction. The product was extracted with AcOEt twice and $\mathrm{CH}_{2} \mathrm{Cl}_{2}$ once, and the combined organic layer was dried over $\mathrm{Na}_{2} \mathrm{SO}_{4}$. The solvent was removed under reduced pressure, and the residue was purified by column chromatography (silica gel, hexane-AcOEt, 7:1 to $5: 1$ to $0: 1)$ to afford $36(191 \mathrm{mg}, 0.417 \mathrm{mmol})$ as a colorless solid in $51 \%$ yield, along with $32(71.6 \mathrm{mg}, 0.23 \mathrm{mmol})$ in $28 \%$ yield. Cyclic carbamate 32 can be recycled (See S-5). IR (KBr): 3313, 2926, 2223, 1679, 1543, 1288, 1167, 1068, 741, $698 \mathrm{~cm}^{-1} ;{ }^{1} \mathrm{H} \mathrm{NMR}\left(\mathrm{CDCl}_{3}\right): \delta=7.40-7.27$ (m, 5H), 6.47 (s, 1H), $5.88(\mathrm{~d}, J=7.4 \mathrm{~Hz}, 1 \mathrm{H}), 5.08$ (s, 2H), 4.64 (d, J=7.4 Hz, 1H), 3.91 (brs, 2H), 3.74 $(\mathrm{dd}, J=8.5,17.0 \mathrm{~Hz}, 1 \mathrm{H}), 3.34(\mathrm{t}, J=5.7 \mathrm{~Hz}, 1 \mathrm{H}), 2.63$ (d, $J=17.3 \mathrm{~Hz}, 1 \mathrm{H}), 2.40$ (dd, $J=8.5,17.3 \mathrm{~Hz}$, $1 \mathrm{H}), 1.55-1.45(\mathrm{~m}, 4 \mathrm{H}), 1.37(\mathrm{~s}, 9 \mathrm{H}), 0.89(\mathrm{t}, J=7.4 \mathrm{~Hz}, 6 \mathrm{H}) \mathrm{ppm} ;{ }^{13} \mathrm{C} \mathrm{NMR}\left(\mathrm{CDCl}_{3}\right): \delta=156.4,156.2$, 143.3, 136.3, 128.4, 128.1, 127.9, 117.6, 111.8, 83.2, 80.2, 75.0, 66.8, 54.1, 49.3, 32.6, 28.2, 26.1, 25.8, 9.4, 9.3 ppm; MS (ESI): $m / z 480\left[\mathrm{M}+\mathrm{Na}^{+}\right]$; HRMS (EI): $m / z$ calcd for $\mathrm{C}_{25} \mathrm{H}_{35} \mathrm{~N}_{3} \mathrm{O}_{5}\left[\mathrm{M}^{+}\right]: 457.2571$. Found: 457.2588; $[\alpha]^{24}{ }_{\mathrm{D}}-48.1\left(c=0.650, \mathrm{CHCl}_{3}\right)$. 
<smiles>CCC(CC)OC1C=C(C#N)CC(NC(C)=O)[C@H]1NC(C)=O</smiles>

36

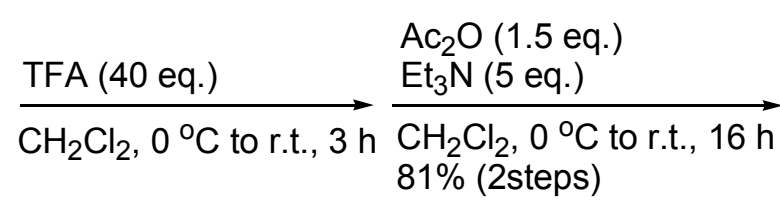
$81 \%$ (2steps)

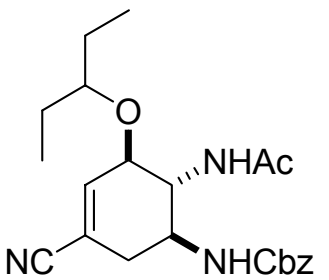

19

To a solution of 36 (130 mg, $0.284 \mathrm{mmol})$ in $\mathrm{CH}_{2} \mathrm{Cl}_{2}(5 \mathrm{~mL})$, TFA (420 $\mu \mathrm{L}, 5.65 \mathrm{mmol}, 20$ equiv) was added at $0{ }^{\circ} \mathrm{C}$. After $1 \mathrm{~h}$, additional TFA (420 $\mathrm{L}, 5.65 \mathrm{mmol}, 20$ equiv) was added at $0{ }^{\circ} \mathrm{C}$, and the mixture was raised to room temperature. After $2 \mathrm{~h}$, TFA was removed in vacuo. The residue was diluted in $\mathrm{CH}_{2} \mathrm{Cl}_{2}(5 \mathrm{~mL})$, to which $\mathrm{Et}_{3} \mathrm{~N}\left(200 \mu \mathrm{L}, 1.43 \mathrm{mmol}, 5\right.$ equiv) and $\mathrm{Ac}_{2} \mathrm{O}$ (40 $\mu \mathrm{L}, 0.43 \mathrm{mmol}, 1.5$ equiv) were added at $0{ }^{\circ} \mathrm{C}$. The mixture was stirred at room temperature for $16 \mathrm{~h}$. After concentration, the residue was purified by column chromatography (silica gel, hexane-AcOEt, 2:1 to 1:1) to afford $19(92.2 \mathrm{mg}$, $0.231 \mathrm{mmol}$ ) as a colorless solid in $81 \%$ yield. IR (KBr): 3335, 2926, 2222, 1690, 1655, 1544, 1293, 1085, 1065, $700 \mathrm{~cm}^{-1} ;{ }^{1} \mathrm{H}$ NMR $\left(\mathrm{CDCl}_{3}\right): \delta=7.42-7.28(\mathrm{~m}, 5 \mathrm{H}), 6.48(\mathrm{~s}, 1 \mathrm{H}), 5.57(\mathrm{~d}, J=9.1 \mathrm{~Hz}, 1 \mathrm{H}), 5.55(\mathrm{~d}$, $J=9.6 \mathrm{~Hz}, 1 \mathrm{H}), 5.12(\mathrm{~d}, J=12.5 \mathrm{~Hz}, 1 \mathrm{H}), 5.05(\mathrm{~d}, J=12.5 \mathrm{~Hz}, 1 \mathrm{H}), 4.12-4.02(\mathrm{~m}, 1 \mathrm{H}), 4.01-3.94$ (m, $1 \mathrm{H}), 3.93-3.83(\mathrm{~m}, 1 \mathrm{H}), 3.37-3.25(\mathrm{~m}, 1 \mathrm{H}), 2.65(\mathrm{dd}, J=4.5,17.6 \mathrm{~Hz}, 1 \mathrm{H}), 2.40(\mathrm{dd}, J=9.1,17.6 \mathrm{~Hz}$, $1 \mathrm{H}), 1.87(\mathrm{~s}, 3 \mathrm{H}), 1.54-1.43(\mathrm{~m}, 4 \mathrm{H}), 0.88(\mathrm{t}, J=7.4 \mathrm{~Hz}, 3 \mathrm{H}), 0.87(\mathrm{t}, J=7.4 \mathrm{~Hz}, 3 \mathrm{H}) \mathrm{ppm} ;{ }^{13} \mathrm{C}$ NMR $\left(\mathrm{CDCl}_{3}\right): \delta=171.2,156.5,143.4,136.2,128.5,128.2,127.9,117.5,111.7,82.7,74.8,66.9,53.2,49.1$, 32.7, 26.0, 25.6, 23.1, 9.4, 9.2 ppm; MS (ESI): $m / z 422\left[\mathrm{M}+\mathrm{Na}^{+}\right]$; HRMS (EI): $\mathrm{m} / z$ calcd for $\mathrm{C}_{22} \mathrm{H}_{29} \mathrm{~N}_{3} \mathrm{O}_{4}$ $\left[\mathrm{M}^{+}\right]$: 399.2153. Found: 399.2147; $[\alpha]^{25}{ }_{\mathrm{D}}-68.5\left(c=0.900, \mathrm{CHCl}_{3}\right)$.

Ethyl (3R,4R,5S)-4-Acetylamino-5-amino-3-(1-ethylpropoxy)cyclohexene-1-carboxylate

(37:

\section{Tamiflu-Free)}<smiles>CCC(CC)OC1C=C(C#N)CC(NC(C)=O)[C@H]1NC(=O)O</smiles>

19

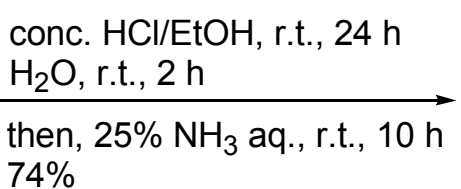
$74 \%$

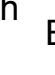$$
\mathrm{tO}_{2} \mathrm{C}
$$

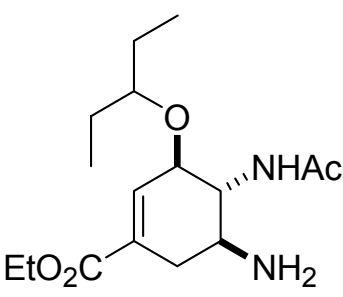

37 $\underset{\text { crystallization }}{\stackrel{\mathrm{H}_{3} \mathrm{PO}_{4}, \mathrm{EtOH}^{5) 6)} \text { 7) }}{\longrightarrow}}$ Tamiflu $^{\circledR}(\mathbf{1})$

(Tamiflu-Free)

A solution of $19(21.2 \mathrm{mg}, 0.053 \mathrm{mmol})$ in conc. $\mathrm{HCl} / \mathrm{EtOH}(5 \mathrm{~mL})$ was stirred at room temperature for $24 \mathrm{~h}$. After concentration of the mixture in vacuo, $\mathrm{H}_{2} \mathrm{O}(100 \mu \mathrm{L})$ in EtOH $(2 \mathrm{~mL})$ were added in order to decompose the imino ester. After $2 \mathrm{~h}, 25 \%$ aqueous $\mathrm{NH}_{3}(2 \mathrm{~mL})$ and $\mathrm{EtOH}(4 \mathrm{~mL})$ were added to the mixture. After $10 \mathrm{~h}$, solvent was removed under reduced pressure. To the residue, $\mathrm{CH}_{2} \mathrm{Cl}_{2}$ and saturated aqueous $\mathrm{NaHCO}_{3}$ were added slowly, and organic layer was separated. The product in water layer was extracted with $\mathrm{CH}_{2} \mathrm{Cl}_{2}$ twice. The combined organic layer was dried over $\mathrm{Na}_{2} \mathrm{SO}_{4}$. Filtration and removal of solvent gave the Tamiflu-free $37(12.2 \mathrm{mg}, 0.039 \mathrm{mmol})$ as a colorless oil in 74\% yield. IR (neat): 3283 , 2963, 2930, 2876, 1715, 1652, 1556, 1373, 1259, 1061, $802 \mathrm{~cm}^{-1} ;{ }^{1} \mathrm{H}$ NMR $\left(\mathrm{CDCl}_{3}\right): \delta=6.73(\mathrm{t}, J=2.0$ $\mathrm{Hz}, 1 \mathrm{H}), 5.61(\mathrm{~d}, J=7.9 \mathrm{~Hz}, 1 \mathrm{H}), 4.25-4.15(\mathrm{~m}, 3 \mathrm{H}), 3.52$ (qlike, $J=10.2 \mathrm{~Hz}, 1 \mathrm{H}), 3.34$ (quintet, $J=5.7$ $\mathrm{Hz}, 1 \mathrm{H}), 3.24$ (td, $J=5.4,10.2 \mathrm{~Hz}, 1 \mathrm{H}), 2.75$ (dd, $J=5.4,18.1 \mathrm{~Hz}, 1 \mathrm{H}), 2.20-2.11(\mathrm{~m}, 1 \mathrm{H}), 2.04(\mathrm{~s}, 3 \mathrm{H})$, 
$1.58-1.44(\mathrm{~m}, 4 \mathrm{H}), 1.29(\mathrm{t}, J=7.4 \mathrm{~Hz}, 3 \mathrm{H}), 0.904(\mathrm{t}, J=7.4 \mathrm{~Hz}, 3 \mathrm{H}), 0.895(\mathrm{t}, J=7.4 \mathrm{~Hz}, 3 \mathrm{H}) \mathrm{ppm} ;{ }^{13} \mathrm{C}$ NMR $\left(\mathrm{CDCl}_{3}\right): \delta=170.9,166.3,137.5,129.5,81.6,74.8,60.8,59.0,49.2,33.6,26.2,25.7,23.7,14.2$, 9.5, 9.3 ppm; MS (ESI): $m / z 313\left[\mathrm{M}+\mathrm{H}^{+}\right]$and $335\left[\mathrm{M}+\mathrm{Na}^{+}\right]$; HRMS (EI): $m / z$ calcd for $\mathrm{C}_{16} \mathrm{H}_{28} \mathrm{~N}_{2} \mathrm{O}_{4}\left[\mathrm{M}^{+}\right]$: 312.2044. Found: 312.2043 ; $[\alpha]^{24}-52.9\left(c=0.610, \mathrm{CHCl}_{3}\right)$. [lit. $\left.[\alpha]_{\mathrm{D}}^{24}-56.2\left(c=0.566, \mathrm{CHCl}_{3}\right)^{4}\right]$

Further conversion from 37 to Tamiflu ${ }^{\circledR}$ (1) was reported in some literatures. ${ }^{5,6,7}$ According to these methods, we performed the $\mathrm{H}_{3} \mathrm{PO}_{4}$ salt formation followed by crystallization to obtain Tamiflu ${ }^{\circledR}(\mathbf{1})^{2}$. All analytical data completely matched with the reported one. ${ }^{5}$

\section{(C) Asymmetric Synthesis of Tamiflu ${ }^{\circledR}$-Optimized Allylic Substitution Route $(1 R, 5 S, 6 S)-6-A z i d o-2-o x 0-4-a z a b i c y c l o[3.3 .1]$ non-7-en-3-one (20)}

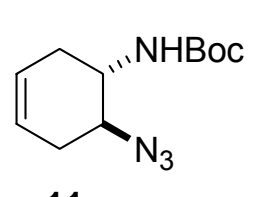

11

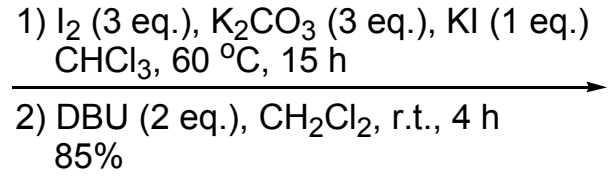
$85 \%$

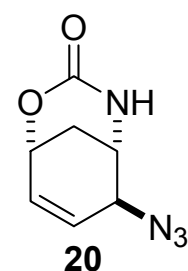

20

To a solution of $11(1.35 \mathrm{~g}, 5.67 \mathrm{mmol})$ in chloroform $(68 \mathrm{ml})$, potassium carbonate $(2.35 \mathrm{~g}, 17.02$ mmol), iodine $(4.32 \mathrm{~g}, 17.02 \mathrm{mmol})$, and potassium iodide $(0.94 \mathrm{~g}, 5.67 \mathrm{mmol})$ were added at $4{ }^{\circ} \mathrm{C}$, and the reaction mixture was warmed to $60{ }^{\circ} \mathrm{C}$. After stirred for $14 \mathrm{~h}$, saturated aqueous $\mathrm{Na}_{2} \mathrm{~S}_{2} \mathrm{O}_{3}(50 \mathrm{ml})$ was added to quench the reaction. The product was extracted with $\mathrm{CH}_{2} \mathrm{Cl}_{2}(20 \mathrm{ml})$ twice. The combined organic layer was washed with brine $(50 \mathrm{ml})$ and dried over $\mathrm{Na}_{2} \mathrm{SO}_{4}$. The solvent was concentrated in vacuo.

To a solution of the residue in chloroform (68 ml), 1,8-diazabicyclo[5.4.0]undec-7-ene (1.7 $\mathrm{ml}, 11.34$ mmol) was added at $4{ }^{\circ} \mathrm{C}$, and the reaction mixture was warmed to room temperature. After stirred for $4 \mathrm{~h}$, the reaction mixture was concentrated in vacuo. The residue was purified by flash column chromatography (EtOAc:hexane $2: 1 \rightarrow 4: 1 \rightarrow$ EtOAc) to afford $20(870.5 \mathrm{mg}, 4.83 \mathrm{mmol})$ as a white solid in $85 \%$ yield. IR (neat) $v_{\max }=3371,2946,2100,1704,1671,1460 \mathrm{~cm}^{-1} ;{ }^{1} \mathrm{H}$ NMR $\left(d_{6}\right.$-DMSO): $\delta=$ 7.52 (brs, $1 \mathrm{H}$ ), 6.28 (dd, $J=6.3,9.8 \mathrm{~Hz}, 1 \mathrm{H}), 6.05$ (dd, $J=5.2,9.8 \mathrm{~Hz}, 1 \mathrm{H}), 4.73-4.69$ (m, $1 \mathrm{H}), 3.97$ (brs, $1 \mathrm{H}$ ), 3.61 (brs, $1 \mathrm{H}), 3.33$ (s, $2 \mathrm{H}) \mathrm{ppm} ;{ }^{13} \mathrm{C}$ NMR $\left(d_{6}\right.$-DMSO): $\delta=151.3,129.8,126.1,65.9,58.0$, 47.1, 22.2 ppm; MS (ESI): $m / z 203\left[\mathrm{M}+\mathrm{Na}^{+}\right]$; HRMS (EI): $m / z$ calcd for $\mathrm{C}_{7} \mathrm{H}_{8} \mathrm{~N}_{4} \mathrm{O}_{2}\left[\mathrm{M}^{+}\right]: 180.0642$. Found: $180.0673 ;[\alpha]^{26}+494.8\left(c=1.02, \mathrm{CHCl}_{3}, 99 \%\right.$ ee $)$.

\footnotetext{
${ }^{4}$ This is an optical rotation value of authentic sample of $\mathbf{3 7}$, which was obtained after extraction from commercial "Tamiflu ${ }^{\circledR}$ capsule 75 (Roche's product)" under basic condition.

5 Rohloff, J. C.; Kent, K. M.; Postich, M. J.; Becker, M. W.; Chapman, H. H.; Kelly, D. E.; Lew, W.; Louie, M. S.; McGee, L.

R.; Prisbe, E. J.; Schultze, L. M.; Yu, R. H.; Zhang, L. J. Org. Chem. 1998, 63, 4545.

6 Iding, H.; Wirz, B; Zutter, U. EP Patent 1,146,036, 2001.

7 Harrington, P. J.; Brown, J. D.; Foderaro, T.; Hughes, R. C. Org. Process Dev. 2004, 8, 86. 
<smiles>N#CC1CC2C=CC1NC(=O)O2</smiles>

20

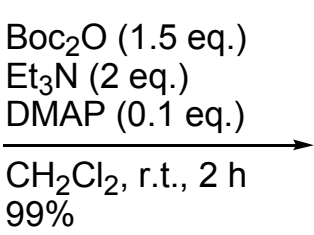

$99 \%$

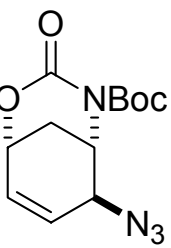

38

To a solution of $20(869.7 \mathrm{mg}, 4.83 \mathrm{mmol})$ in dichloromethane $(30 \mathrm{ml})$, triethylamine $(1.3 \mathrm{ml}, 9.66$ $\mathrm{mmol})$, 4-dimethylaminopyridine (59 $\mathrm{mg}, 0.483 \mathrm{mmol})$, and di-tert-butyl dicarbonate (1.58 g, $7.24 \mathrm{mmol})$ in dichloromethane $(18 \mathrm{ml})$ were added at $4{ }^{\circ} \mathrm{C}$, and the reaction mixture was warmed to room temperature. After stirred for $2 \mathrm{~h}$, water $(40 \mathrm{ml})$ was added to quench the reaction. The product was extracted with $\mathrm{CH}_{2} \mathrm{Cl}_{2}(20 \mathrm{ml})$ twice. The combined organic layer was washed with brine $(50 \mathrm{ml})$, dried over $\mathrm{Na}_{2} \mathrm{SO}_{4}$, and concentrated in vacuo. The residue was purified by flash column chromatography (EtOAc:hexane 1:2 $\rightarrow 1: 1)$ to afford $38\left(1.34 \mathrm{~g}, 4.78 \mathrm{mmol}\right.$ ) as a white solid in $99 \%$ yield. IR (neat) $v_{\max }$ $=2980,2101,1793,1747 \mathrm{~cm}^{-1} ;{ }^{1} \mathrm{H}$ NMR $\left(\mathrm{CDCl}_{3}\right): \delta=6.28(\mathrm{dd}, J=6.3,9.7 \mathrm{~Hz}, 1 \mathrm{H}), 6.01(\mathrm{dd}, J=4.6$, 9.7 Hz, $1 \mathrm{H}), 4.71-4.67$ (m, $1 \mathrm{H}), 4.54-4.49$ (m, $1 \mathrm{H}), 4.09-4.04$ (m, $1 \mathrm{H}), 2.25-2.20$ (m, $1 \mathrm{H}), 2.16-2.10$ $(\mathrm{m}, 1 \mathrm{H}), 1.54(\mathrm{~s}, 9 \mathrm{H}) \mathrm{ppm} ;{ }^{13} \mathrm{C} \mathrm{NMR}\left(\mathrm{CDCl}_{3}\right): \delta=151.1,147.6,129.0,126.9,84.6,67.0,56.9,52.2$, 27.8, $23.1 \mathrm{ppm}$; MS (ESI): $\mathrm{m} / z 303\left[\mathrm{M}+\mathrm{Na}^{+}\right]$and $203\left[\mathrm{M}-\mathrm{Boc}+\mathrm{H}+\mathrm{Na}^{+}\right]$; HRMS (FAB): $m / z$ calcd for $\mathrm{C}_{12} \mathrm{H}_{17} \mathrm{~N}_{4} \mathrm{O}_{4}\left[\mathrm{M}+\mathrm{H}^{+}\right]$: 281.1244. Found: 281.1247; $[\alpha]^{25}+361.0\left(c=0.97, \mathrm{CHCl}_{3}, 99 \%\right.$ ee $)$.

tert-Butyl (1R,5S,6S)-6-(Acetylamino)-3-0xo-2-oxa-4-azabicyclo[3.3.1]non-7-en-4-carboxylate (21)
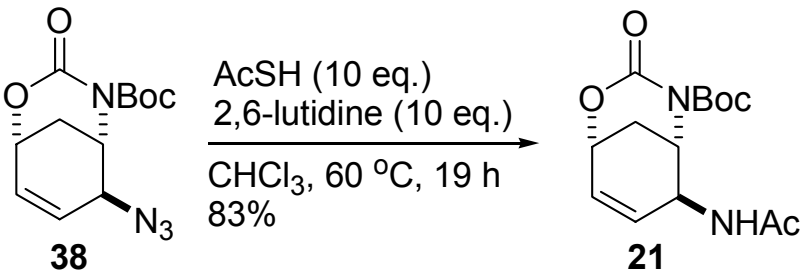

To a solution of $\mathbf{3 8}(1.25 \mathrm{~g}, 4.46 \mathrm{mmol})$ in chloroform $(25 \mathrm{ml}), 2,6$-lutidine $(5.2 \mathrm{ml}, 44.58 \mathrm{mmol})$ and thioacetic acid ( $3 \mathrm{ml}, 44.58 \mathrm{mmol})$ were added at room temperature, and the reaction mixture was warmed to $60{ }^{\circ} \mathrm{C}$. After stirred for $19 \mathrm{~h}$, the reaction mixture was concentrated in vacuo, and the residue was purified by flash column chromatography (EtOAc:hexane 2:1 $\rightarrow$ EtOAc $\rightarrow$ EtOAc:MeOH 10:1) to afford 21 (1.10 g, $3.71 \mathrm{mmol})$ as a white solid in 83\% yield. IR (neat) $v_{\max }=3336,2979,1776,1735$, 1675, $1541 \mathrm{~cm}^{-1} ;{ }^{1} \mathrm{H}$ NMR $\left(\mathrm{CDCl}_{3}\right): \delta=6.22(\mathrm{~d}, J=8.6 \mathrm{~Hz}, 1 \mathrm{H}), 6.14(\mathrm{dd}, J=6.3,9.5 \mathrm{~Hz}, 1 \mathrm{H}), 5.91(\mathrm{dd}$, $J=4.6,9.5 \mathrm{~Hz}, 1 \mathrm{H}), 4.72-4.67(\mathrm{~m}, 1 \mathrm{H}), 4.60-4.55(\mathrm{~m}, 1 \mathrm{H}), 4.52-4.48(\mathrm{~m}, 1 \mathrm{H}), 2.27-2.21(\mathrm{~m}, 1 \mathrm{H})$, 2.12-2.06 (m, $1 \mathrm{H}), 1.97(\mathrm{~s}, 3 \mathrm{H}), 1.56(\mathrm{~s}, 9 \mathrm{H}) \mathrm{ppm}$ (Its chemical shifts depend on the substrate concentration); ${ }^{13} \mathrm{C} \mathrm{NMR}\left(\mathrm{CDCl}_{3}\right): \delta=169.3,150.6,148.7,129.7,127.1,84.8,67.3,52.4,47.0,27.6$, 23.3, $23.0 \mathrm{ppm}$; MS (ESI) : $m / z 319\left[\mathrm{M}+\mathrm{Na}^{+}\right]$and $219\left[\mathrm{M}-\mathrm{Boc}+\mathrm{H}+\mathrm{Na}^{+}\right]$; HRMS (EI): $m / z$ calcd for $\mathrm{C}_{14} \mathrm{H}_{20} \mathrm{~N}_{2} \mathrm{O}_{5}\left[\mathrm{M}^{+}\right]$: 296.1367 Found: 296.1370; $[\alpha]^{26}+209.4(c=0.86, \mathrm{MeOH}, 99 \%$ ee $)$. 


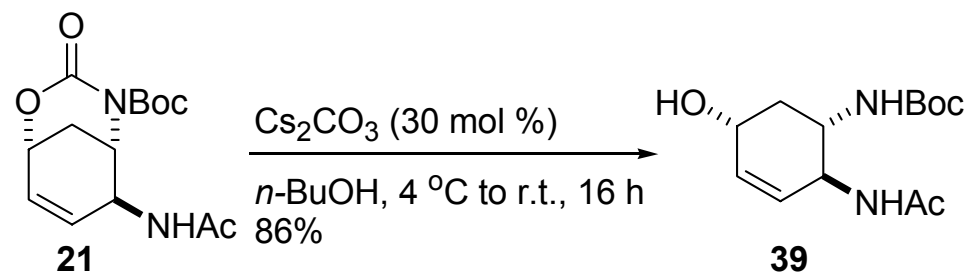

To a solution of $21(933 \mathrm{mg}, 3.15 \mathrm{mmol})$ in $n$-butanol $(79 \mathrm{ml})$, cesium carbonate $(308 \mathrm{mg}, 0.95 \mathrm{mmol})$ was added at $4{ }^{\circ} \mathrm{C}$ and the reaction mixture was warmed to room temperature. After stirring for $16 \mathrm{~h}$, the reaction mixture was concentrated in vacuo directly. The residue was purified by flash column chromatography (EtOAc $\rightarrow$ EtOAc:MeOH 10:1 $\rightarrow$ 5:1) to afford 39 (733 mg, $2.71 \mathrm{mmol}$ ) as a white solid in $86 \%$ yield. IR $(\mathrm{KBr}) v_{\max }=3303,2973,2451,1685,1646,1560 \mathrm{~cm}^{-1} ;{ }^{1} \mathrm{H}$ NMR $\left(d_{4}-\mathrm{MeOH}\right): \delta=$ 5.60 (brd, $J=10.1 \mathrm{~Hz}, 1 \mathrm{H}), 5.47$ (brd, $J=10.1 \mathrm{~Hz}, 1 \mathrm{H}), 4.40-4.31$ (m, $2 \mathrm{H}), 3.58-3.50$ (m, $1 \mathrm{H}$ ), 2.23-2.19 (m, $1 \mathrm{H}), 1.92(\mathrm{~s}, 3 \mathrm{H}), 1.61-1.53(\mathrm{~m}, 1 \mathrm{H}), 1.42(\mathrm{~s}, 9 \mathrm{H}) \mathrm{ppm} ;{ }^{13} \mathrm{C} \mathrm{NMR}\left(d_{4}-\mathrm{MeOH}\right): \delta=173.4$, $158.1,134.4,129.4,80.2,67.5,52.5,52.1,39.7,28.7,22.7$ ppm; MS （ESI） : $m / z 293\left[\mathrm{M}+\mathrm{Na}^{+}\right]$; HRMS (FAB): $m / z$ calcd for $\mathrm{C}_{13} \mathrm{H}_{23} \mathrm{~N}_{2} \mathrm{O}_{4}\left[\mathrm{M}+\mathrm{H}^{+}\right]: 271.1652$. Found: $271.1652 ;[\alpha]^{26}{ }_{\mathrm{D}}+88.0(c=0.46, \mathrm{MeOH}$, 99\% ee).

tert-Butyl [(1S,2S)-2-(Acetylamino)-5-oxocyclohex-3-en-1-yl]carbamate (22)<smiles>CC(C)(C)OC(=O)N[C@H]1C=C[C@@H](O)C[C@H]1NC(=O)O</smiles>

39

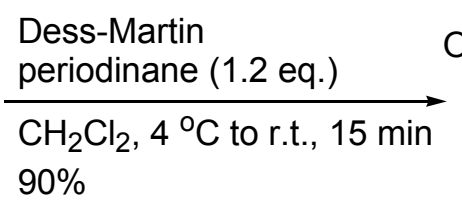
$90 \%$

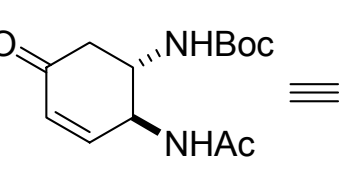

$22(99 \%$ ee $)$

To a slurry of $39(797 \mathrm{mg}, 2.95 \mathrm{mmol})$ in dichloromethane $(59 \mathrm{ml})$, Dess-Martin periodinane $(1.5 \mathrm{~g}$, $3.54 \mathrm{mmol}$ ) was added at $4{ }^{\circ} \mathrm{C}$. After stirring for $10 \mathrm{~min}$, the reaction mixture was warmed to room temperature. The reaction mixture was stirred for $15 \mathrm{~min}$, and saturated aqueous $\mathrm{Na}_{2} \mathrm{~S}_{2} \mathrm{O}_{3}(30 \mathrm{ml})$ was poured into the reaction mixture. The product was extracted with $\mathrm{CH}_{2} \mathrm{Cl}_{2}(30 \mathrm{ml})$ twice. The combined organic layer was washed with saturated aqueous $\mathrm{NaHCO}_{3}(30 \mathrm{ml})$ and brine $(30 \mathrm{ml})$ sequentially, and dried over $\mathrm{Na}_{2} \mathrm{SO}_{4}$. The solvent was concentrated, and the residue was purified by flash column chromatography (EtOAc:hexane 4:1 $\rightarrow$ EtOAc $\rightarrow$ EtOAc:MeOH 10:1) to afford 22 including a small amount of a reagent residue. The crude 22 was washed with EtOAc $(20 \mathrm{ml})$ in order to remove the reagent residue to afford pure $22(506 \mathrm{mg}, 1.89 \mathrm{mmol}, 99 \%$ ee) in 64\% yield. The filtrate was concentrated in vacuo and purified by flash column chromatography (EtOAc:hexane 4:1 $\rightarrow$ EtOAc $\rightarrow$ EtOAc:MeOH 10:1) to afford $22(281 \mathrm{mg})$. The obtained $22(281 \mathrm{mg})$ was recrystallized with EtOAc (3 $\mathrm{ml}$ ) to afford pure 22 (209 mg, $0.78 \mathrm{mmol}, 99 \%$ ee ) in 26\% yield. Enone 22 was obtained in total $90 \%$ yield. The optical purity of $\mathbf{2 2}$ was determined by HPLC analysis (Chiralpak OD-H, 2-propanol/hexane $1 / 20$, flow $1.0 \mathrm{ml} / \mathrm{min}$, detection at $254 \mathrm{~nm}$; $\mathrm{t}_{\mathrm{R}} 10.4 \mathrm{~min}$ (minor) and $15.1 \mathrm{~min}$ (major). $\mathrm{IR}(\mathrm{KBr}) v_{\max }=$ 3350, 3288, 2973, 1704, 1659, $1551 \mathrm{~cm}^{-1} ;{ }^{1} \mathrm{H}$ NMR $\left(\mathrm{CDCl}_{3}\right): \delta=6.80(\mathrm{dd}, J=10.1,2.0 \mathrm{~Hz}, 1 \mathrm{H}), 6.51$ (brd, $J=6.7 \mathrm{~Hz}, 1 \mathrm{H}), 6.07(\mathrm{dd}, J=10.1,2.0 \mathrm{~Hz}, 1 \mathrm{H}), 4.86(\mathrm{brd}, J=8.9 \mathrm{~Hz}, 1 \mathrm{H}), 4.71-4.66(\mathrm{~m}, 1 \mathrm{H})$, 4.12-4.02 (m, $1 \mathrm{H}), 2.78$ (dd, $J=16.0,4.0 \mathrm{~Hz}, 1 \mathrm{H}), 2.41$ (dd, $J=16.0,13.8 \mathrm{~Hz}, 1 \mathrm{H}), 2.01(\mathrm{~s}, 3 \mathrm{H}), 1.44$ (s, $9 \mathrm{H}) \mathrm{ppm} ;{ }^{13} \mathrm{C} \mathrm{NMR}\left(\mathrm{CDCl}_{3}\right): \delta=195.9,170.8,156.4,150.4,129.8,80.6,53.8,51.0,43.4,28.2,23.1$ 
ppm; MS (ESI): $m / z 291\left[\mathrm{M}+\mathrm{Na}^{+}\right]$; HRMS (FAB): $m / z$ calcd for $\mathrm{C}_{13} \mathrm{H}_{21} \mathrm{~N}_{2} \mathrm{O}_{4}\left[\mathrm{M}+\mathrm{H}^{+}\right]: 269.1496$. Found: 269.1495; $[\alpha]_{\mathrm{D}}^{24}+122.1(c=0.78, \mathrm{MeOH}, 99 \%$ ee $)$.

tert-Butyl [(1S,5S,6R)-6-(Acetylamino)-3-cyano-5-hydroxycyclohex-3-en-1-yl]carbamate (24)

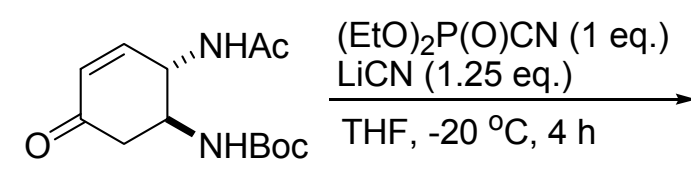

22

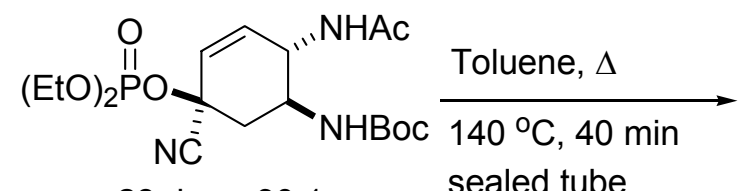

$23 \mathrm{dr}=>30: 1$

estimated structure

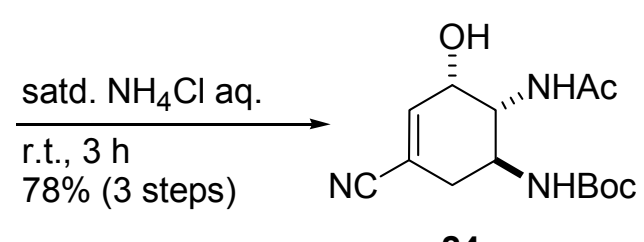

24

28

To a solution of $22(206 \mathrm{mg}, 0.768 \mathrm{mmol})$ in tetrahydrofuran $(15 \mathrm{ml})$, diethylphosphoryl cyanide (116 $\mu 1,0.768 \mathrm{mmol}$ ) was added at room temperature. The reaction mixture was cooled to $-20{ }^{\circ} \mathrm{C}$. Every 1 hour, lithium cyanide (total $31.7 \mathrm{mg}, 0.96 \mathrm{mmol})^{3}$ was added in 3 divided doses. After stirred for $4 \mathrm{~h}$, saturated aqueous ammonium chloride $(10 \mathrm{ml})$ and water $(10 \mathrm{ml})$ was poured into the reaction mixture at $-20{ }^{\circ} \mathrm{C}$. The product was extracted with EtOAc $(20 \mathrm{ml})$ twice. The combined organic layer was washed with brine (30 ml) and dried over $\mathrm{Na}_{2} \mathrm{SO}_{4}$. The solvent was concentrated in vacuo.

Toluene $(19 \mathrm{ml})$ was added to 23 , and the reaction mixture was heated at $140{ }^{\circ} \mathrm{C}$ in a sealed tube. After $40 \mathrm{~min}$, the reaction mixture was cooled to room temperature. Subsequently, saturated aqueous ammonium chloride $(10 \mathrm{ml})$ and tetrahydrofuran $(10 \mathrm{ml})$ were added to the reaction mixture, and the heterogeneous mixture was stirred for $3 \mathrm{~h}$ vigorously. Finally, aqueous $1 \mathrm{M} \mathrm{NaOH}(10 \mathrm{ml})$ was added and the product was extracted with EtOAc $(20 \mathrm{ml})$ twice. The combined organic layer was washed with brine $(30 \mathrm{ml})$ and dried over $\mathrm{Na}_{2} \mathrm{SO}_{4}$. The solvent was concentrated in vacuo. The residue was purified by flash column chromatography (EtOAc:hexane 2:1 $\rightarrow$ 4:1 $\rightarrow$ EtOAc $\rightarrow$ EtOAc:MeOH 40:1) to afford 24 (177 $\mathrm{mg}, 0.596 \mathrm{mmol})$ as amorphous in $78 \%$ yield.

\section{tert-Butyl $\{(1 S, 2 S, 5 R)-2-($ Acetylamino)-5-cyano-5-[(diethoxyphosphoryl)oxy]cyclohex-3-en-1-yl $\}$}

carbamate $(23){ }^{1} \mathrm{H}$ NMR $\left(\mathrm{CDCl}_{3}\right): \delta=6.64(\mathrm{~d}, J=8.0 \mathrm{~Hz}, 1 \mathrm{H}), 6.01(\mathrm{~d}, J=10.3 \mathrm{~Hz}, 1 \mathrm{H}), 5.91(\mathrm{~d}, J=$ $10.3 \mathrm{~Hz}, 1 \mathrm{H}), 5.28(\mathrm{~d}, J=8.0 \mathrm{~Hz}, 1 \mathrm{H}), 4.52$ (brd, $J=8.6,9.0 \mathrm{~Hz}, 1 \mathrm{H}), 4.19-4.10$ (m, $4 \mathrm{H}), 3.87$ (brd, $J$ $=18.9,9.0 \mathrm{~Hz}, 1 \mathrm{H}), 2.84(\mathrm{~d}, J=12.6 \mathrm{~Hz}, 1 \mathrm{H}), 2.21(\mathrm{dd}, J=12.6,12.6 \mathrm{~Hz}, 1 \mathrm{H}), 1.96(\mathrm{~s}, 3 \mathrm{H}), 1.40$ (s, 9 $\mathrm{H}), 1.33(\mathrm{t}, J=7.0 \mathrm{~Hz}, 6 \mathrm{H}) \mathrm{ppm}$.

24: IR (neat) $v_{\max }=3338,2979,2224,1685,1654,1534 \mathrm{~cm}^{-1} ;{ }^{1} \mathrm{H}$ NMR $\left(\mathrm{CDCl}_{3}\right): \delta=6.75(\mathrm{~d}, J=9.2 \mathrm{~Hz}$, $1 \mathrm{H}), 6.61(\mathrm{dd}, J=5.2,2.3 \mathrm{~Hz}, 1 \mathrm{H}), 5.42(\mathrm{~d}, J=9.2 \mathrm{~Hz}, 1 \mathrm{H}), 4.68$ (brd, $J=8.5 \mathrm{~Hz}, 1 \mathrm{H}), 4.20$ (brs, $1 \mathrm{H}$ ), 4.18-4.07 (m, $1 \mathrm{H}), 4.01-3.93(\mathrm{~m}, 1 \mathrm{H}), 2.69(\mathrm{dd}, J=17.8,5.5 \mathrm{~Hz}, 1 \mathrm{H}), 2.34-2.25(\mathrm{~m}, 1 \mathrm{H}), 2.00(\mathrm{~s}, 3 \mathrm{H})$, 1.39 (s, $9 \mathrm{H}) \mathrm{ppm} ;{ }^{13} \mathrm{C} \mathrm{NMR}\left(\mathrm{CDCl}_{3}\right): \delta=171.4,157.1,141.6,117.4,114.5,80.6,65.9,52.8,45.3,34.0$, 28.3, 23.0 ppm; MS (ESI): $m / z 318\left[\mathrm{M}+\mathrm{Na}^{+}\right]$; HRMS (FAB): $m / z$ calcd for $\mathrm{C}_{14} \mathrm{H}_{22} \mathrm{~N}_{3} \mathrm{O}_{4}\left[\mathrm{M}+\mathrm{H}^{+}\right]$: 296.1605. Found: $296.1640 ;[\alpha]^{25}+37.6\left(c=0.76, \mathrm{CHCl}_{3}, 99 \%\right.$ ee $)$. 


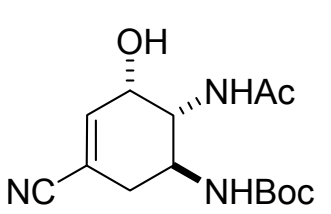

24

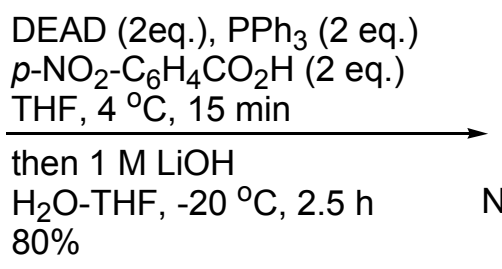

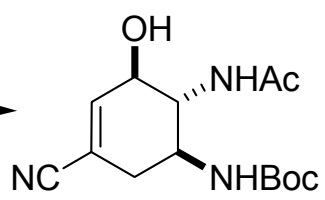

25

To a solution of 24 (282 mg, $0.956 \mathrm{mmol}$ ), triphenylphosphine (501 $\mathrm{mg}, 1.91 \mathrm{mmol})$, and p-nitorobenzoic acid $(320 \mathrm{mg}, 1.91 \mathrm{mmol})$ in tetrahydrofuran $(19 \mathrm{ml})$, DEAD (40\% in toluene, $0.87 \mathrm{ml}$, $1.91 \mathrm{mmol}$ ) was added at $4{ }^{\circ} \mathrm{C}$. After stirring for $15 \mathrm{~min}$, the reaction mixture was cooled to $-20{ }^{\circ} \mathrm{C}$. To the reaction mixture, aqueous $1 \mathrm{M}$ lithium hydroxide $(3.8 \mathrm{ml}, 3.82 \mathrm{mmol})$ was added. After stirring at the same temperature for $2.5 \mathrm{~h}$, the product was extracted with EtOAc $(20 \mathrm{ml})$ twice. The combined organic layer was washed with brine $(30 \mathrm{ml})$ and dried over $\mathrm{Na}_{2} \mathrm{SO}_{4}$. The solvent was concentrated in vacuo. The residue was purified by flash column chromatography (EtOAc:hexane 4:1 $\rightarrow$ EtOAc $\rightarrow$ EtOAc:MeOH 20:1) to afford $25(227 \mathrm{mg}, 0.769 \mathrm{mmol})$ as a white solid in $80 \%$ yield. IR (KBr) $v_{\max }=3322,2979,2225$, 1683, 1625, $1571 \mathrm{~cm}^{-1} ;{ }^{1} \mathrm{H}$ NMR $\left(\mathrm{CDCl}_{3}\right): \delta=7.12(\mathrm{~d}, J=5.2 \mathrm{~Hz}, 1 \mathrm{H}), 6.49(\mathrm{~s}, 1 \mathrm{H}), 5.01(\mathrm{~d}, J=2.3 \mathrm{~Hz}$, $1 \mathrm{H}), 4.82(\mathrm{~d}, J=8.0 \mathrm{~Hz}, 1 \mathrm{H}), 4.28-4.21(\mathrm{~m}, 1 \mathrm{H}), 3.92-3.81(\mathrm{~m}, 1 \mathrm{H}), 3.78-3.70(\mathrm{~m}, 1 \mathrm{H}), 2.63(\mathrm{dd}, J=$ 16.9, $5.0 \mathrm{~Hz}, 1 \mathrm{H}), 2.32-2.22(\mathrm{~m}, 1 \mathrm{H}), 2.00(\mathrm{~s}, 3 \mathrm{H}), 1.44(\mathrm{~s}, 9 \mathrm{H}) \mathrm{ppm} ;{ }^{13} \mathrm{C} \mathrm{NMR}\left(\mathrm{CDCl}_{3}\right): \delta=173.6$,

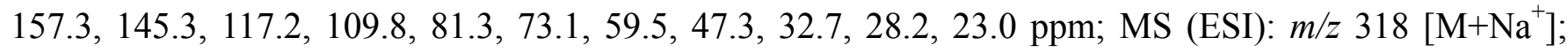
HRMS (FAB): $m / z$ calcd for $\mathrm{C}_{14} \mathrm{H}_{22} \mathrm{~N}_{3} \mathrm{O}_{4}\left[\mathrm{M}+\mathrm{H}^{+}\right]: 296.1605$. Found: $296.1602 ;[\alpha]^{27}{ }_{\mathrm{D}}-89.1(c=0.69$, $\mathrm{MeOH}, 99 \%$ ee).

tert-Butyl [(1R,2S,6S)-7-Acetyl-4-cyano-7-azabicyclo[4.1.0]hept-4-en-2-yl]carbamate (26)<smiles>CC(C)(C)N[C@H]1CC(C#N)=CC(O)[C@H]1NC(C)(C)C</smiles>

25

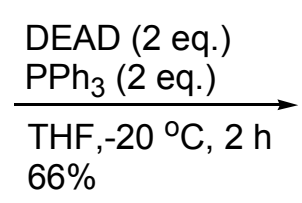

$66 \%$

To a solution of triphenylphosphine $(414 \mathrm{mg}, 1.58 \mathrm{mmol})$ in tetrahydrofran $(7.9 \mathrm{~mL})$, DEAD $(40 \%$ in toluene, $0.72 \mathrm{~mL}, 1.58 \mathrm{mmol})$ was added at $-20{ }^{\circ} \mathrm{C}$. Then, $25(234 \mathrm{mg}, 0.79 \mathrm{mmol})$ in THF $(2.5 \mathrm{~mL})$ was added to the reaction mixture dropwise for $15 \mathrm{~min}$ at the same temperature. After stirring for $1 \mathrm{~h}$, the reaction mixture was concentrated in vacuo. The residue was purified by flash column chromatography twice [first (removal of triphenylphosphine oxide); EtOAc:hexane 4:1 $\rightarrow$ EtOAc $\rightarrow$ EtOAc:MeOH 20:1, second (removal of the residue derived from DEAD); $\mathrm{Et}_{2} \mathrm{O}$ :hexane 3:1 $\rightarrow$ 5:1] to afford 26 (144 $\mathrm{mg}, 0.521 \mathrm{mmol}$ ) as an amorphous in $66 \%$ yield. IR (neat) $v_{\max }=3322,2978,2218,1703,1525 \mathrm{~cm}^{-1}$; ${ }^{1} \mathrm{H} \mathrm{NMR}\left(\mathrm{CDCl}_{3}\right): \delta=6.88(\mathrm{dd}, J=3.5,3.7 \mathrm{~Hz}, 1 \mathrm{H}), 4.54(\mathrm{brs}, 2 \mathrm{H}), 3.12-3.04(\mathrm{~m}, 2 \mathrm{H}), 2.53(\mathrm{brd}, J=$ $17.0 \mathrm{~Hz}, 1 \mathrm{H}), 2.35$ (brd, $J=17.0,1 \mathrm{H}), 2.13(\mathrm{~s}, 3 \mathrm{H}), 1.43(\mathrm{~s}, 9 \mathrm{H}) \mathrm{ppm} ;{ }^{13} \mathrm{C} \mathrm{NMR}\left(\mathrm{CDCl}_{3}\right): \delta=180.8$,

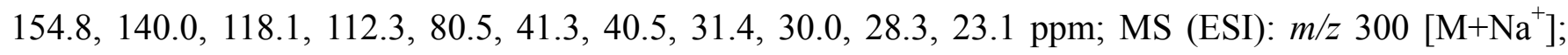
HRMS (FAB): $m / z$ calcd for $\mathrm{C}_{14} \mathrm{H}_{20} \mathrm{~N}_{3} \mathrm{O}_{3}\left[\mathrm{M}+\mathrm{H}^{+}\right]$: 278.1499. Found: $278.1500 ;[\alpha]^{23}{ }_{\mathrm{D}}-104.0(c=0.69$, $\mathrm{CHCl}_{3}, 99 \%$ ee). 
tert-Butyl [(1S,5R,6R)-6-(Acetylamino)-3-cyano-5-(1-ethylpropoxy)cyclohex-3-en-1-yl]carbamate $(27)^{2}$

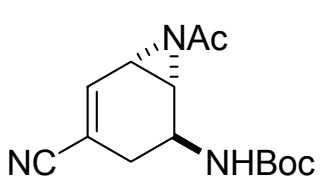

26

$$
\begin{aligned}
& \mathrm{BF}_{3} \cdot \mathrm{OEt}_{2}(1.5 \text { eq. }) \\
& \underset{\mathrm{MS} 5 \mathrm{~A}}{\text { 3-pentanol, }-20^{\circ} \mathrm{C}, 5 \mathrm{~h}}
\end{aligned}
$$$$
\mathrm{NC}
$$

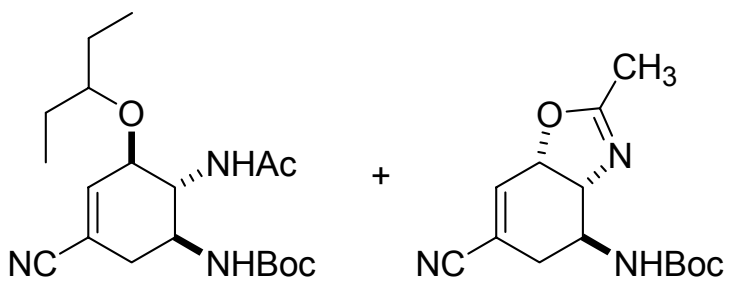

$2913 \%$

(judging from NMR analysis)

To a slurry of $26(119.3 \mathrm{mg}, 0.430 \mathrm{mmol})$ and activated molecular sieves $5 \mathrm{~A}$ (430 $\mathrm{mg})$ in 3-pentanol (4.3 mL), $\mathrm{BF}_{3} \cdot \mathrm{OEt}_{2}(1 \mathrm{M}$ in 3-pentanol, $0.65 \mathrm{~mL}, 0.645 \mathrm{mmol})$ was added dropwise for $15 \mathrm{~min}$ at $-20{ }^{\circ} \mathrm{C}$. After stirred for $1 \mathrm{~h}$, water $(10 \mathrm{ml})$ was poured into the reaction mixture at $-20{ }^{\circ} \mathrm{C}$. The product was extracted with EtOAc $(15 \mathrm{ml})$ twice. The combined organic layer was washed with brine $(15 \mathrm{ml})$ and dried over $\mathrm{Na}_{2} \mathrm{SO}_{4}$. The solvent was concentrated in vacuo. The residue was purified by flash column chromatography (EtOAc:hexane $1: 2 \rightarrow 2: 3 \rightarrow 1: 1)$ to afford $27(88.1 \mathrm{mg}, 0.241 \mathrm{mmol})^{2}$ as a white solid in $56 \%$ yield. $[\alpha]^{26}-99.6\left(c=0.58, \mathrm{CHCl}_{3}, 99 \%\right.$ ee $)$. [lit. $\left.[\alpha]^{21}{ }_{\mathrm{D}}-108.1\left(c=0.660, \mathrm{CHCl}_{3}\right)\right]^{2}$.

tert-Butyl [(3aR,4S,7aS)-6-Cyano-2-methyl-3a,4,5,7a-tetrahydro-1,3-benzoxazol-4-yl]carbamate (29): IR (neat) $v_{\max }=3326,2977,2221,1705,1671,1524 \mathrm{~cm}^{-1} ;{ }^{1} \mathrm{H}$ NMR $\left(\mathrm{CDCl}_{3}\right): \delta=6.64($ brs, $1 \mathrm{H})$, 4.92 (brd, $J=8.6 \mathrm{~Hz}, 1 \mathrm{H}), 4.82(\mathrm{~d}, J=6.9 \mathrm{~Hz}, 1 \mathrm{H}), 4.12(\mathrm{dd}, J=8.6,8.6 \mathrm{~Hz}, 1 \mathrm{H}), 3.61$ (brs, $1 \mathrm{H}), 2.79$ (brd, $J=16.6 \mathrm{~Hz}, 1 \mathrm{H}), 2.24-2.17(\mathrm{~m}, 1 \mathrm{H}), 1.98(\mathrm{~d}, J=1.2 \mathrm{~Hz}, 3 \mathrm{H}), 1.43(\mathrm{~s}, 9 \mathrm{H}) \mathrm{ppm} ;{ }^{13} \mathrm{C} \mathrm{NMR}$ $\left(\mathrm{CDCl}_{3}\right): \delta=166.2,155.3,138.4,117.4,115.4,80.2,74.1,66.4,49.5,30.0,28.3,14.1 \mathrm{ppm}$; MS (ESI): $m / z 300\left[\mathrm{M}+\mathrm{Na}^{+}\right]$; HRMS (FAB): $m / z$ calcd for $\mathrm{C}_{14} \mathrm{H}_{20} \mathrm{~N}_{3} \mathrm{O}_{3}\left[\mathrm{M}+\mathrm{H}^{+}\right]: 278.1499$. Found: 278.1500 .

Ethyl (3R,4R,5S)-4-Acetylamino-5-amino-3-(1-ethylpropoxy)cyclohexene-1-carboxylate (37:

\section{Tamiflu-Free)}

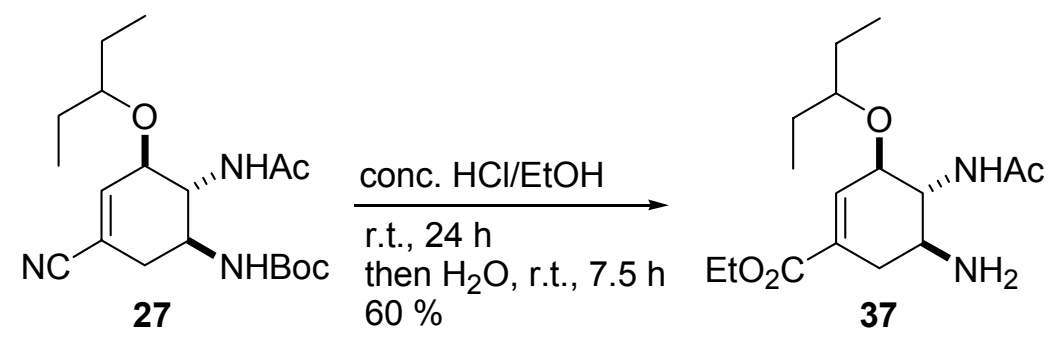

The solution of $27(22.6 \mathrm{mg}, 0.062 \mathrm{mmol})$ in conc. $\mathrm{HCl} / \mathrm{EtOH}(2.5 \mathrm{ml})$ was stirred at room temperature for $24 \mathrm{~h}$. After cooling to $4{ }^{\circ} \mathrm{C}$, water was added to decompose the imino ester. The reaction mixture was warmed to room temperature and stirred for $7.5 \mathrm{~h}$. The reaction mixture was washed with $\mathrm{CH}_{2} \mathrm{Cl}_{2}(10 \mathrm{ml})$. To the water layer, aqueous $2 \mathrm{M} \mathrm{NaOH}$ was added slowly with stirring till $\mathrm{pH}=10$. The product was extracted with $\mathrm{CH}_{2} \mathrm{Cl}_{2}(15 \mathrm{ml})$ four times, and the combined organic layer was dried over $\mathrm{Na}_{2} \mathrm{SO}_{4}$. The solvent was concentrated in vacuo to afford $37(11.5 \mathrm{mg}, 0.037 \mathrm{mmol})$ as a colorless oil in $60 \%$ yield. $[\alpha]_{D}^{25}-56.6\left(c=0.995, \mathrm{CHCl}_{3}\right) .\left[\right.$ lit. $\left.[\alpha]^{24}-56.2\left(c=0.566, \mathrm{CHCl}_{3}\right)^{4}\right]$ 
(D) Estimation of the Allylic Phosphate (28) Structure
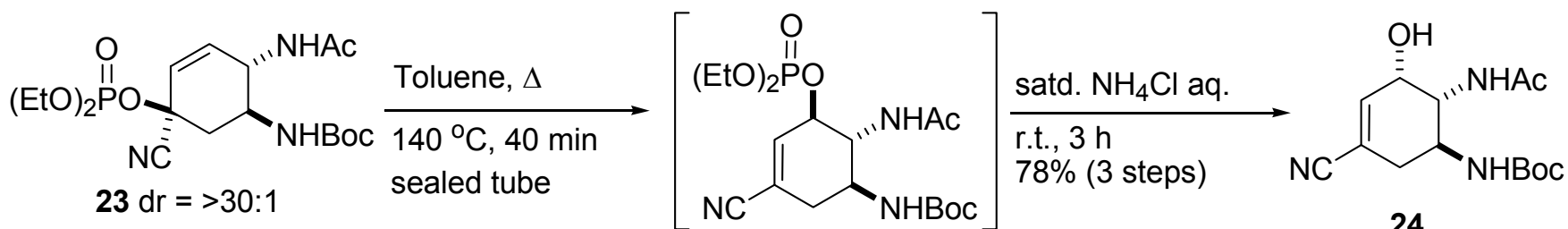

estimated structure

24

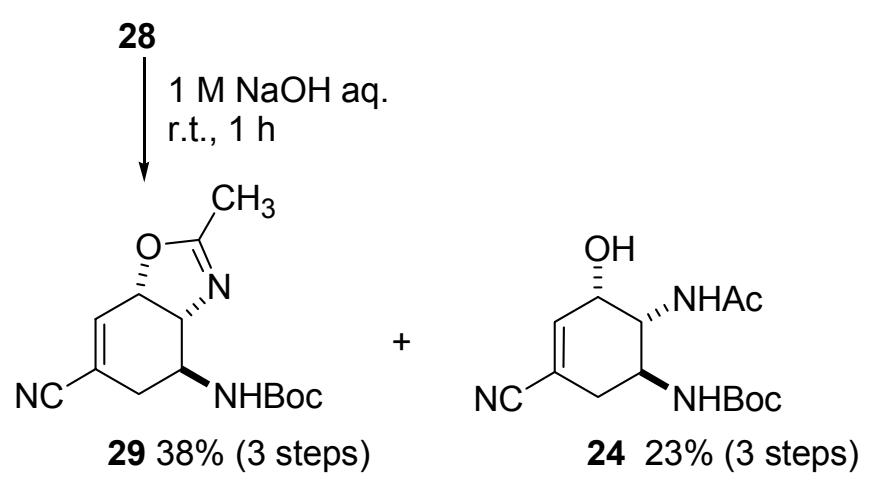

As mentioned in the manuscript, this reaction $(\mathbf{2 3} \rightarrow \mathbf{2 4})$ was considered to proceed via the allylic phosphonate 28. At first, this thermal reaction was studied in order to synthesize oxazoline 29. A solution of 23 in toluene was heated at $140{ }^{\circ} \mathrm{C}$ in a sealed tube for $1 \mathrm{~h}$. After $\mathbf{2 3}$ was consumed completely on TLC analysis, the product was extracted and purified by flash column chromatography. Surprisingly, the $\alpha$-allylic alcohol 24, not the desired oxazoline 29, was isolated as a major product. From this preliminary result, it was expected that this reaction mechanism might be different from the case of 15 .

According to the literature ${ }^{8}$, it is known that $\beta$-allyl- $\alpha$-cyanophosphate rearranges to afford $\beta$-allyl- $\gamma$-cyano phosphate in a supra-facial fashion under thermal conditions. Based on the precedent, we estimated that this reaction proceeded via $\beta$-allyl phosphonate $\mathbf{2 8}$ as an intermediate. Although the isolation of $\mathbf{2 8}$ was tried, the trials were failed due to its lability. Thus, the reaction mixture was directly analyzed by ESI-MS. The peak of $454\left[\mathbf{2 8}+\mathrm{Na}^{+}\right]$was cleanly found even after $\mathbf{2 3}$ was consumed completely (The consumption of $\mathbf{2 3}$ was confirmed by TLC and ${ }^{1} \mathrm{H}$ NMR analysis). Therefore, this peak was estimated to be derived from $\beta$-allyl phosphonate 28 .

When quenching the thermal rearrangement under basic conditions (for example, aqueous $1 \mathrm{M} \mathrm{NaOH}$ ), the acetamide group was activated, and the oxazoline $\mathbf{2 9}$ was produced as a major product through an $\mathrm{S}_{\mathrm{N}} 2$ fashion, along with $\alpha$-allylic alcohol $\mathbf{2 4}$ (the downward arrow from $\mathbf{2 8}$ in the above scheme). The oxazoline 29 was stable, and was not converted to $\alpha$-allylic alcohol 24 at room temperature under basic conditions. Therefore, the $\alpha$-allylic alcohol 24 can be considered to generate through a substitution reaction of 28 by a hydroxy anion. On the other hand, the $\alpha$-allylic alcohol 24 was produced as the major product from 28 through an $\mathrm{S}_{\mathrm{N}} 2$ fashion by the activation of phosphate group under weakly acidic conditions (for example, saturated aqueous $\mathrm{NH}_{4} \mathrm{Cl}$ ) in good yield (the rightward arrow from 28).

\footnotetext{
${ }^{8}$ Kurihara, T.; Miki, M.; Yoneda, R.; Harusawa, S. Chem. Pharm. Bull. 1986, 34, 2747. 


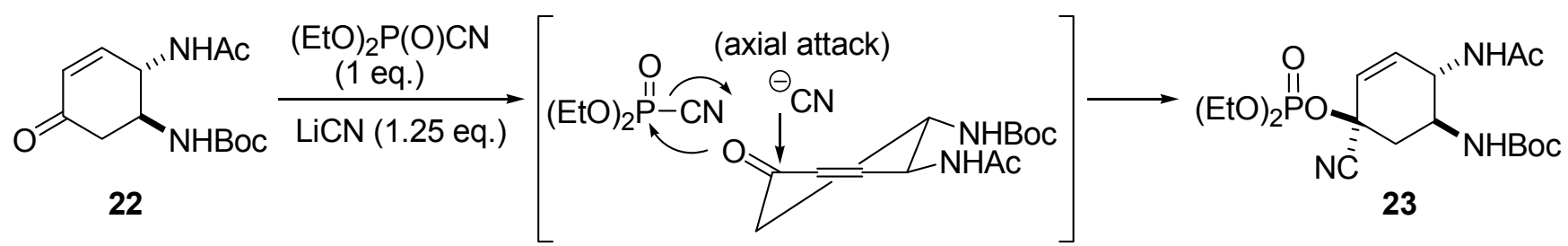

It is considered that the cyanide anion attacks enone $\mathbf{2 2}$ from the axial direction due to the small size of cyanide and the bulkiness of diethyl phosphate. Therefore, the structure of $\mathbf{2 3}$ was considered as the above figure. It was difficult to determine the stereochemistry of $\mathbf{2 3}$ by NOE experiments.

(E) Comparison of ${ }^{1} \mathrm{H}$ NMR Spectra of Tamiflu-Free (37)

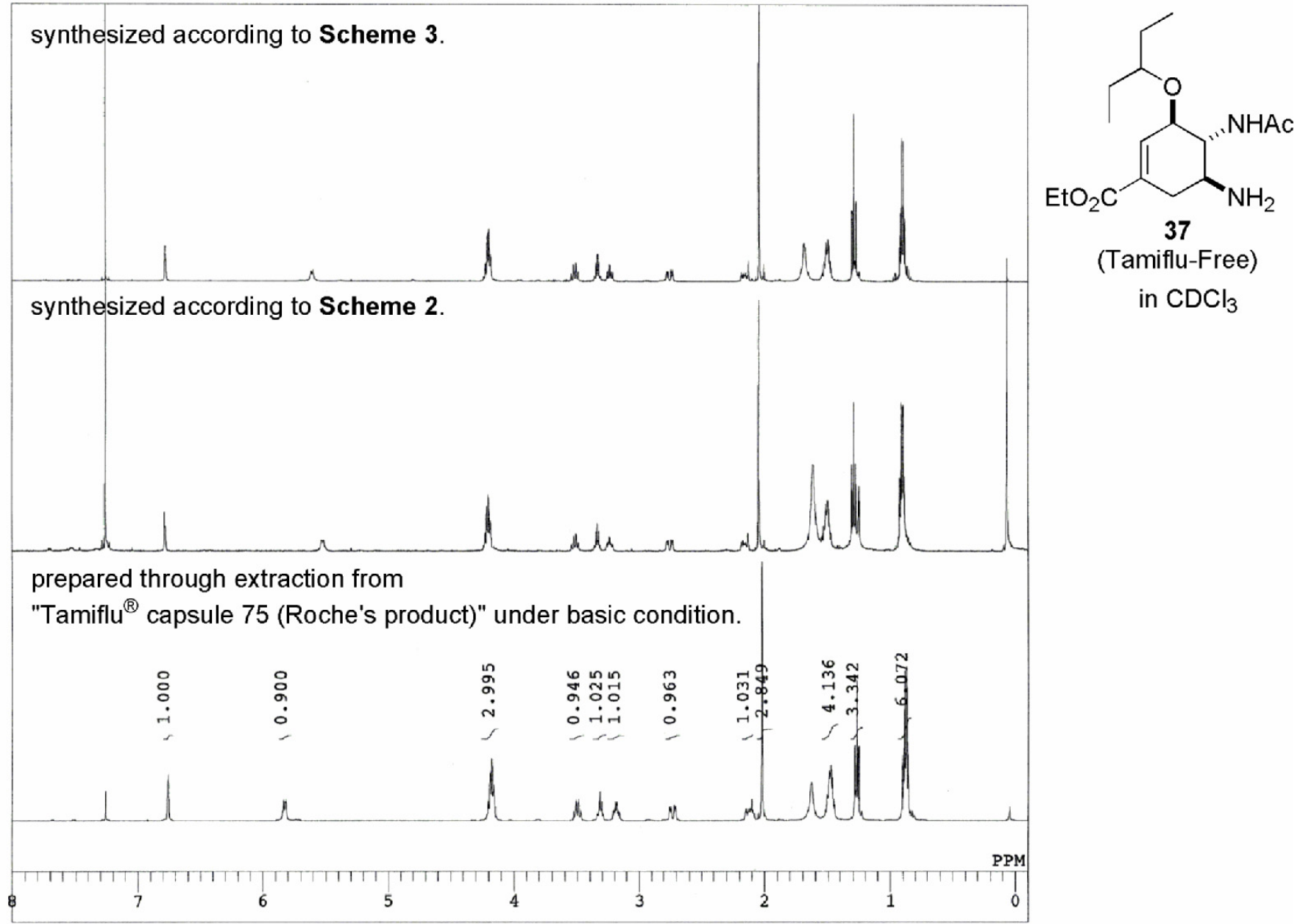


吕

웜 웅

国

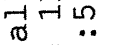

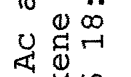

46

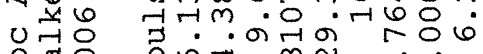

\% . य⿱㇒廾幺小

नं०

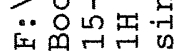

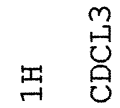

요

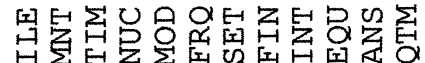

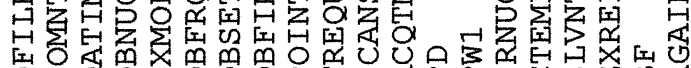

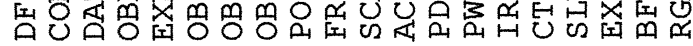

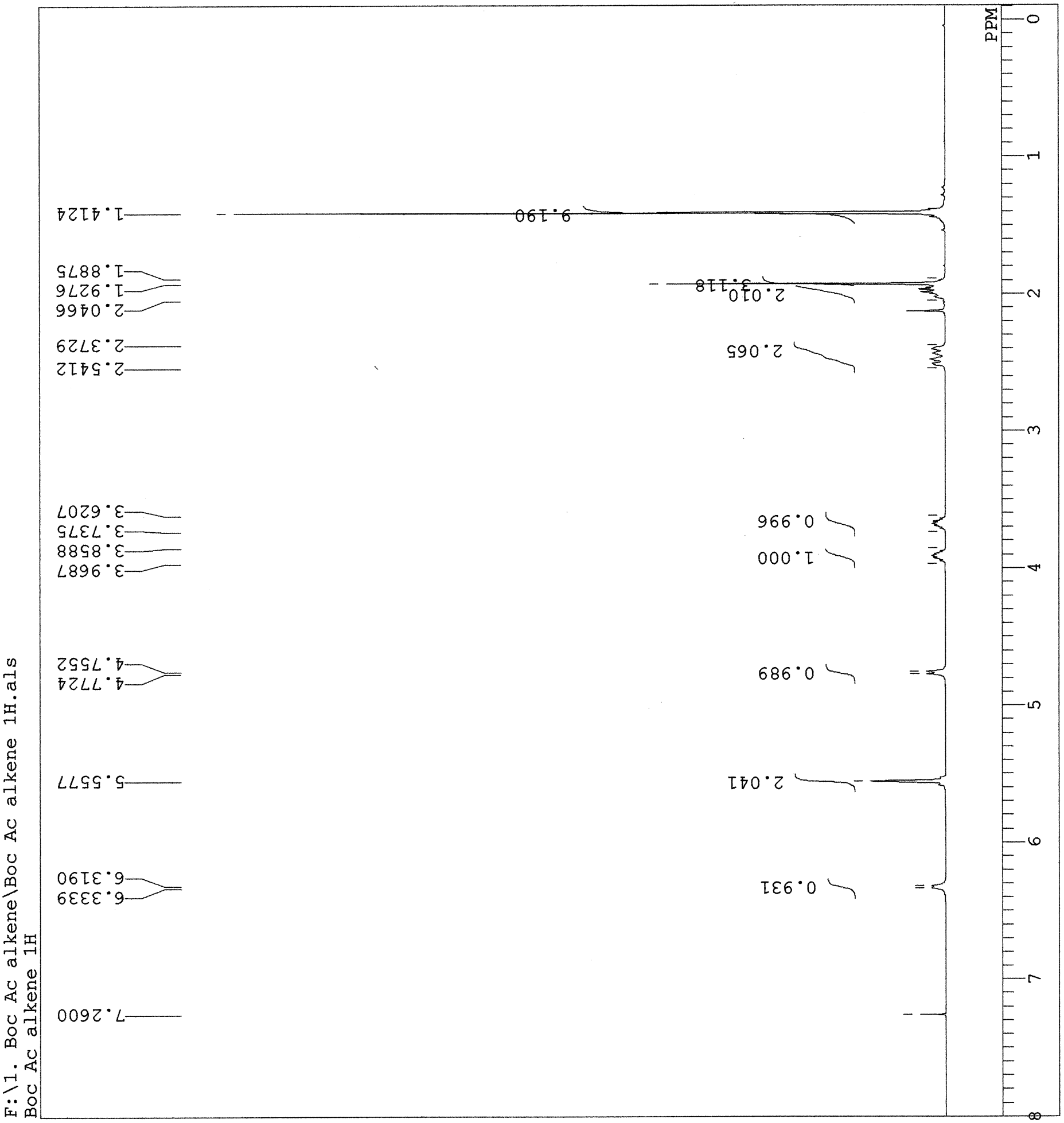




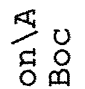

苟足

苟

$\rightarrow-\rightarrow \stackrel{\infty}{m}$

( $)$

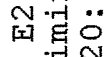

$4-7$

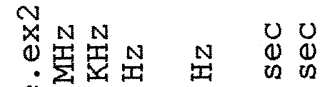
कmmorm नें

4N

岃되유

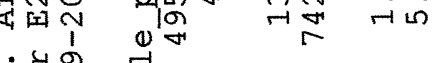

लं क्षे क्ष

mi

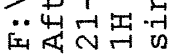

出苞

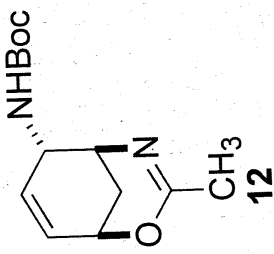

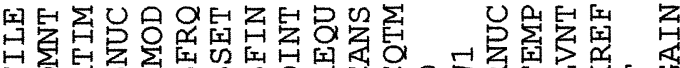

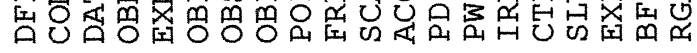

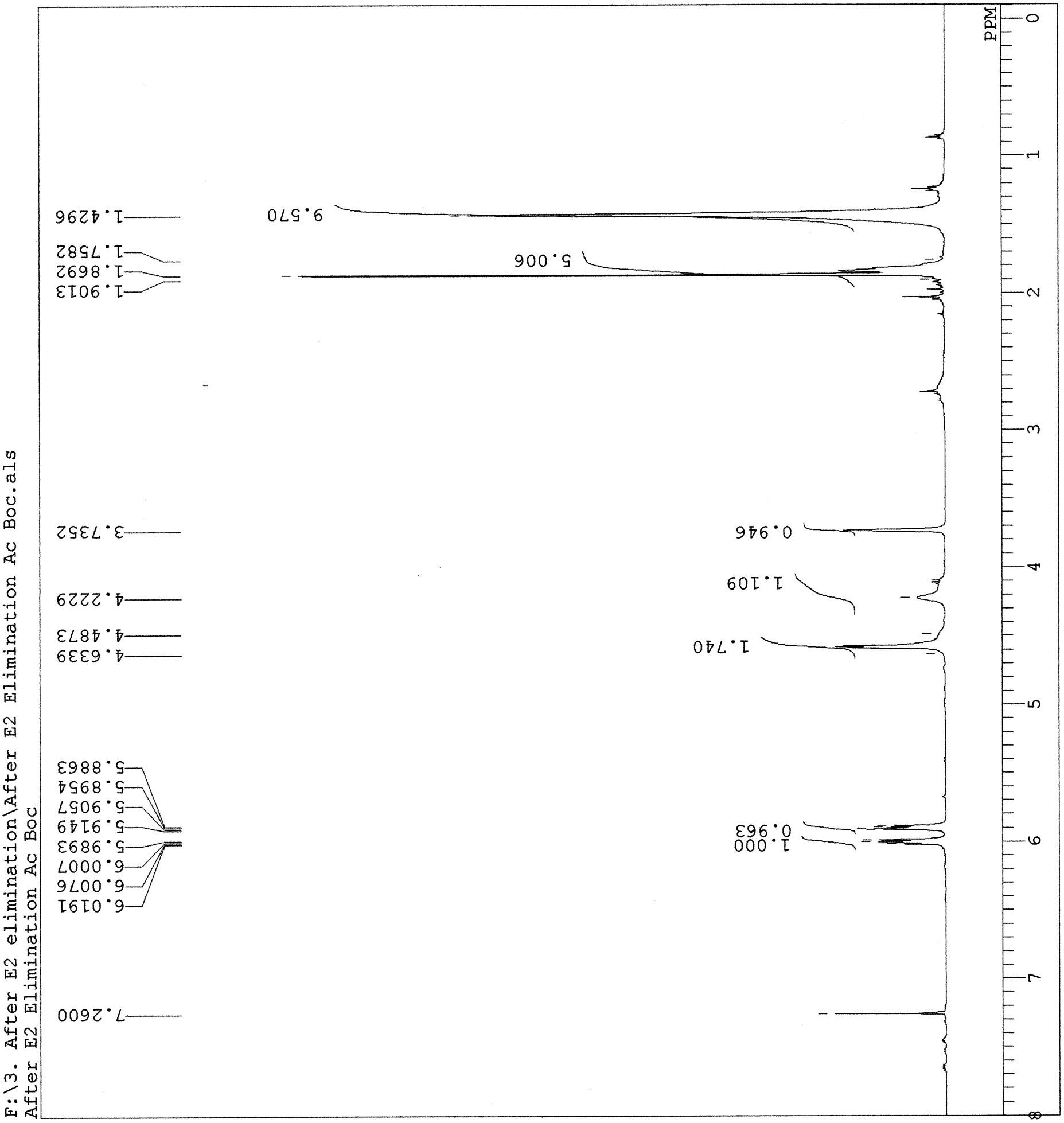


$\stackrel{2}{\rightarrow} \stackrel{0}{-1}$

웜

त्व $\ddot{n}$

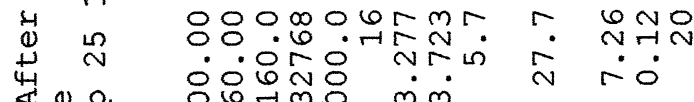
\& मंन थ

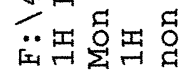

录

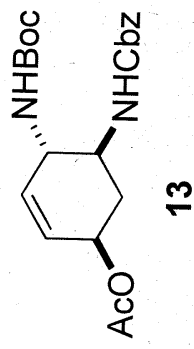

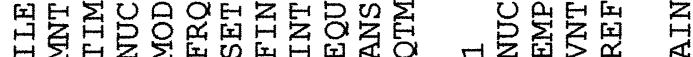

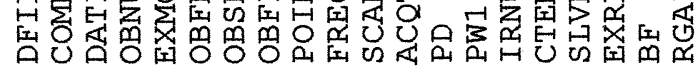

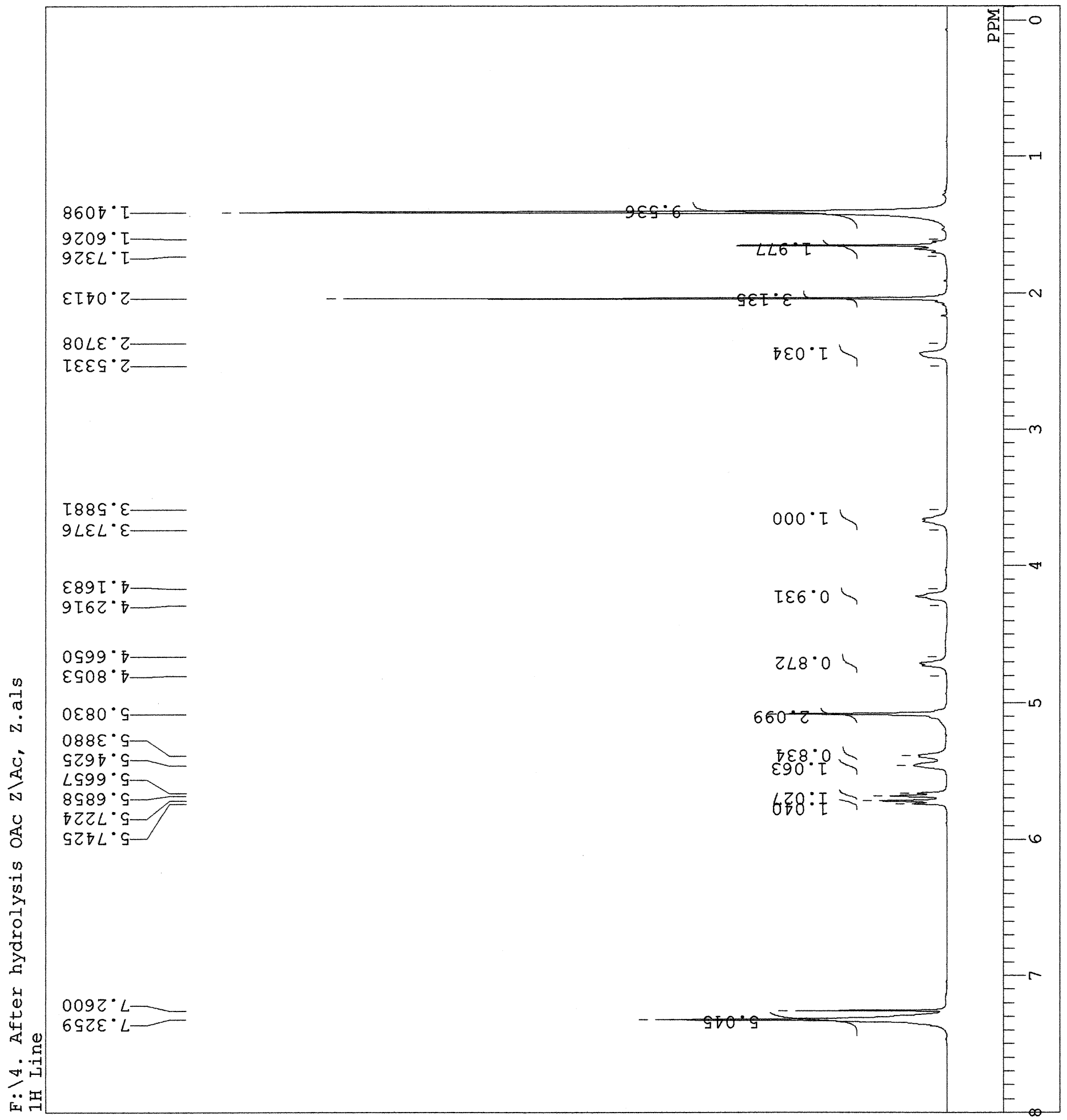




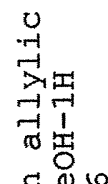

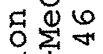

$\underset{\pi}{-1} \frac{1}{d}$

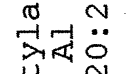

武

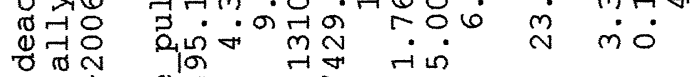

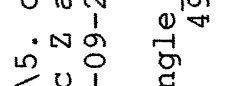

ר...

出 兑

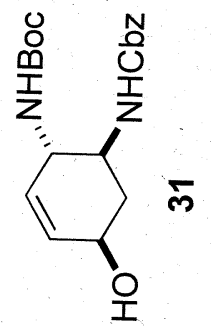

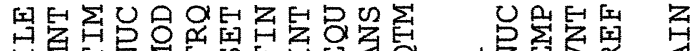

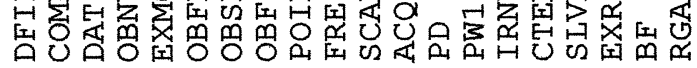

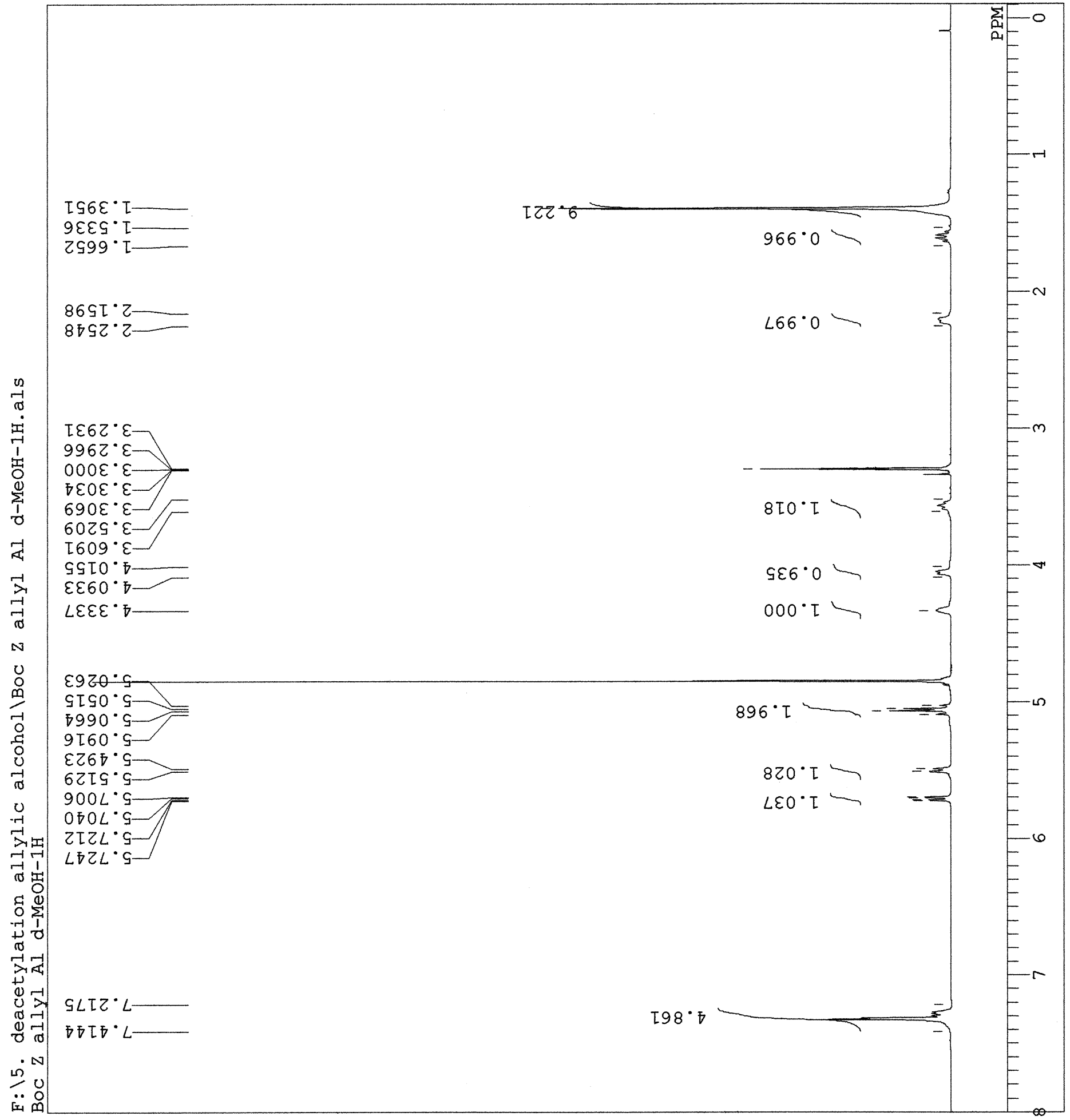


$\stackrel{7}{\because}$

$\ddot{n} N$

O

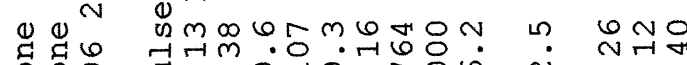

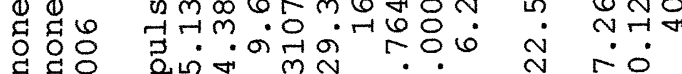
द्व घ्व

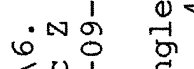
닝 欮命学

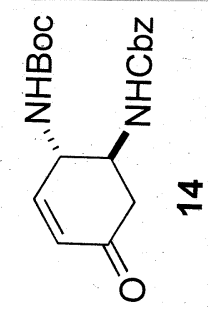

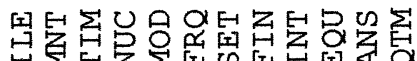

只毕留

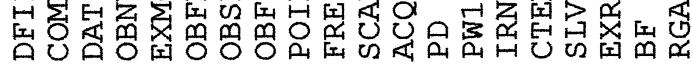

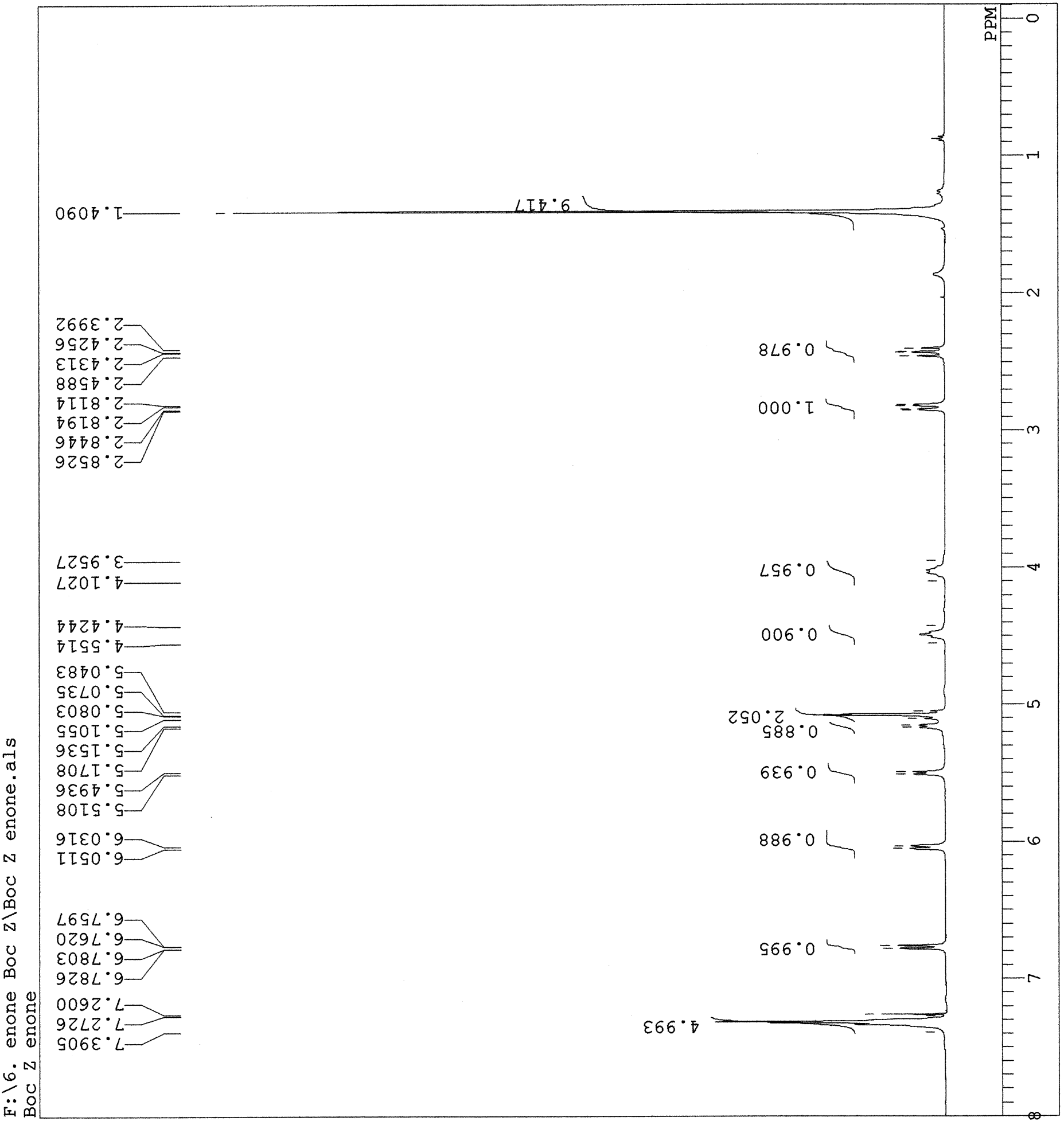




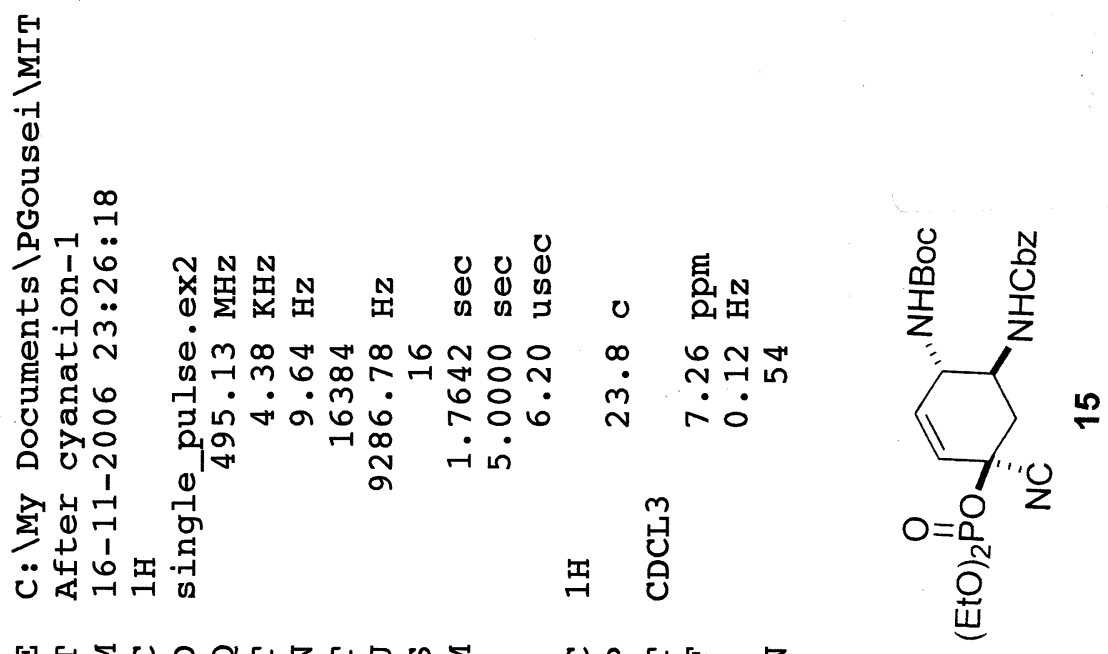

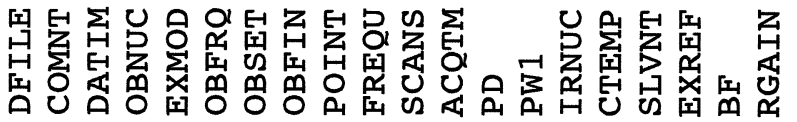
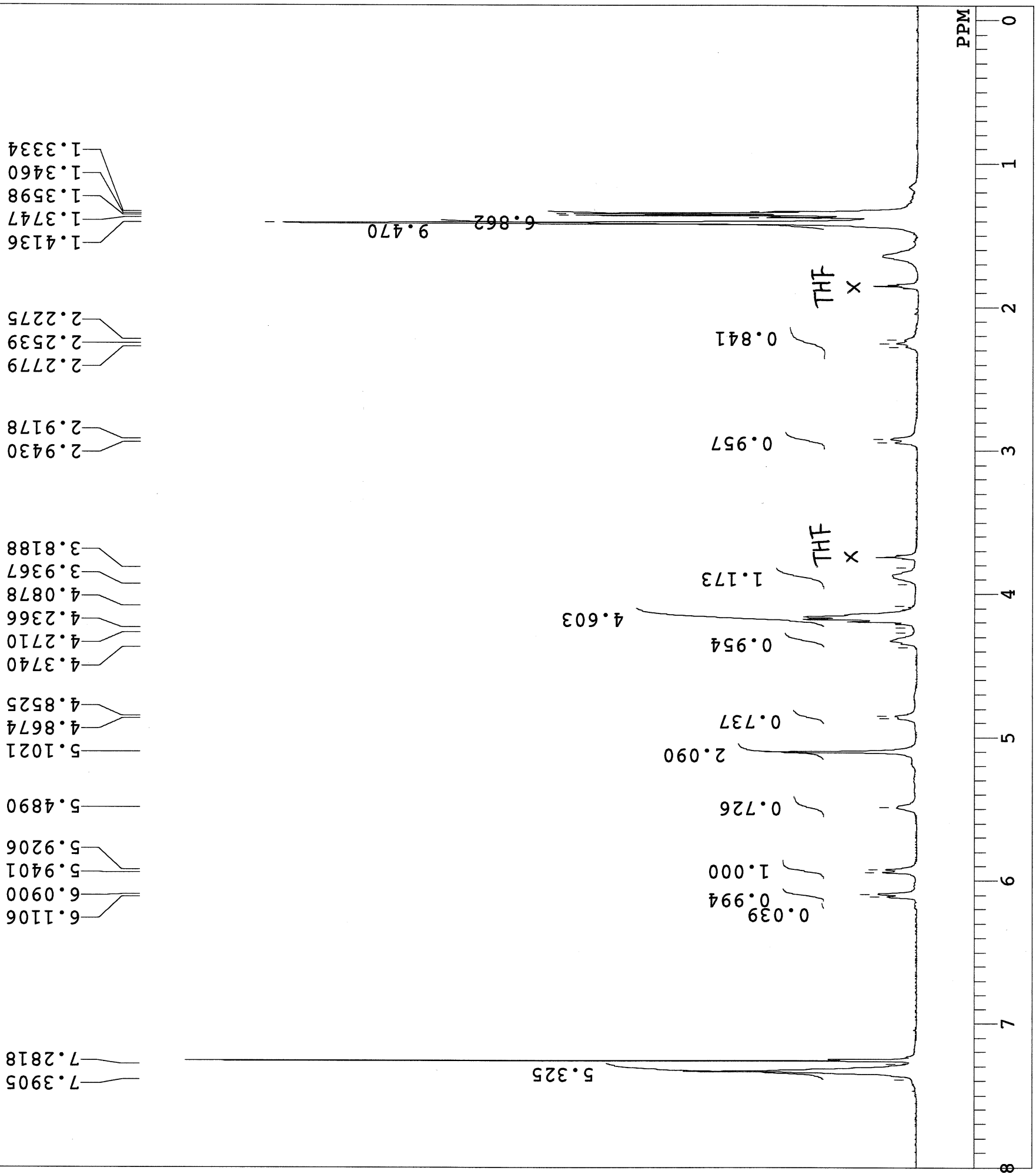
告

苋受

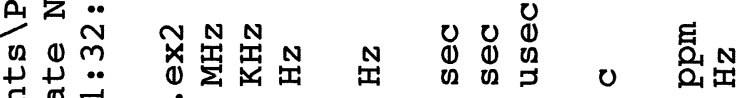

退

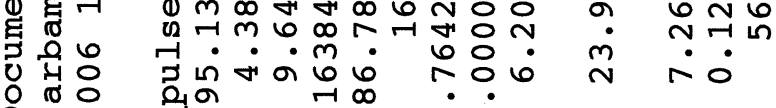

ค ป 仓

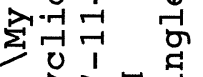

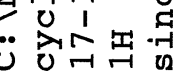

出 藏

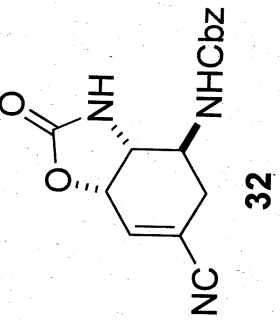

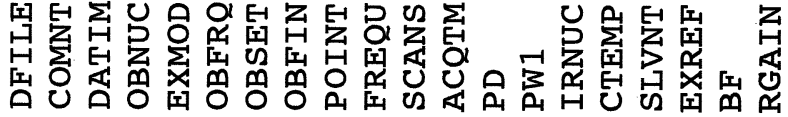

$\angle 9 S^{\circ}[$

โ $\varepsilon \varepsilon \cdot \tau$

乙৪६ђ・て

$0859^{\circ} Z$

$\tau \angle 99^{\circ} Z$

与ع $69^{\circ} Z$

SIOL' $Z$

乙6SL・E

Ђ89L・ $\varepsilon$

$\varepsilon \subseteq L L \cdot \varepsilon$

Ђ๐ $8 L^{\circ} \varepsilon$

$6 \varsigma 86^{\circ} \varepsilon$

$8676^{\circ} \nabla$

$\varepsilon 乙 \angle 0^{\circ} \mathrm{s}$

$0 L G I \cdot G$

$9826 \cdot 5$

$\varepsilon \angle \angle 9^{\circ} 9$

$0092^{\circ} L$

โ80 $\varepsilon^{\circ} L$

$08 โ 7^{\circ} L$ i

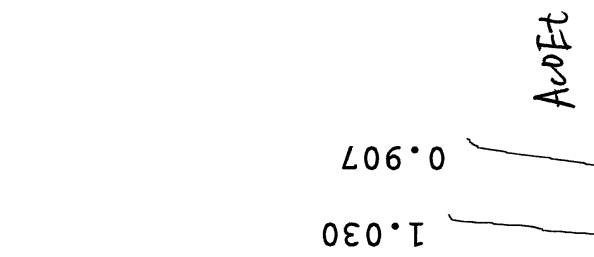

IS $6^{\circ} 0$

$000^{\circ} \mathrm{I}$

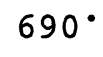

$898^{\circ} 0$
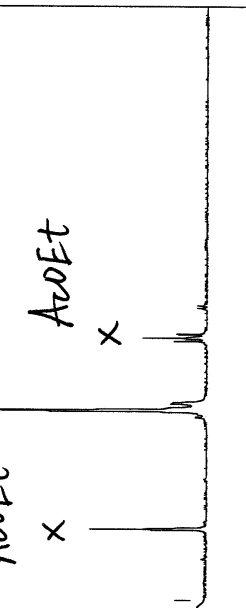

$-\infty$
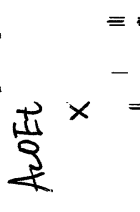

$\sum$

i v 


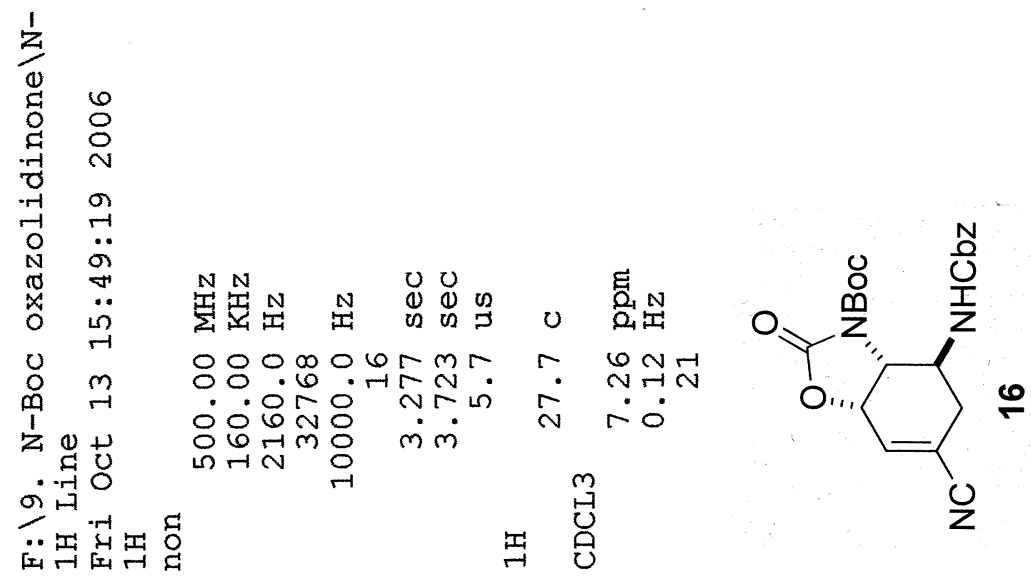

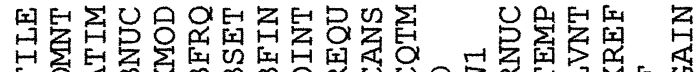

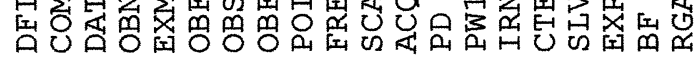

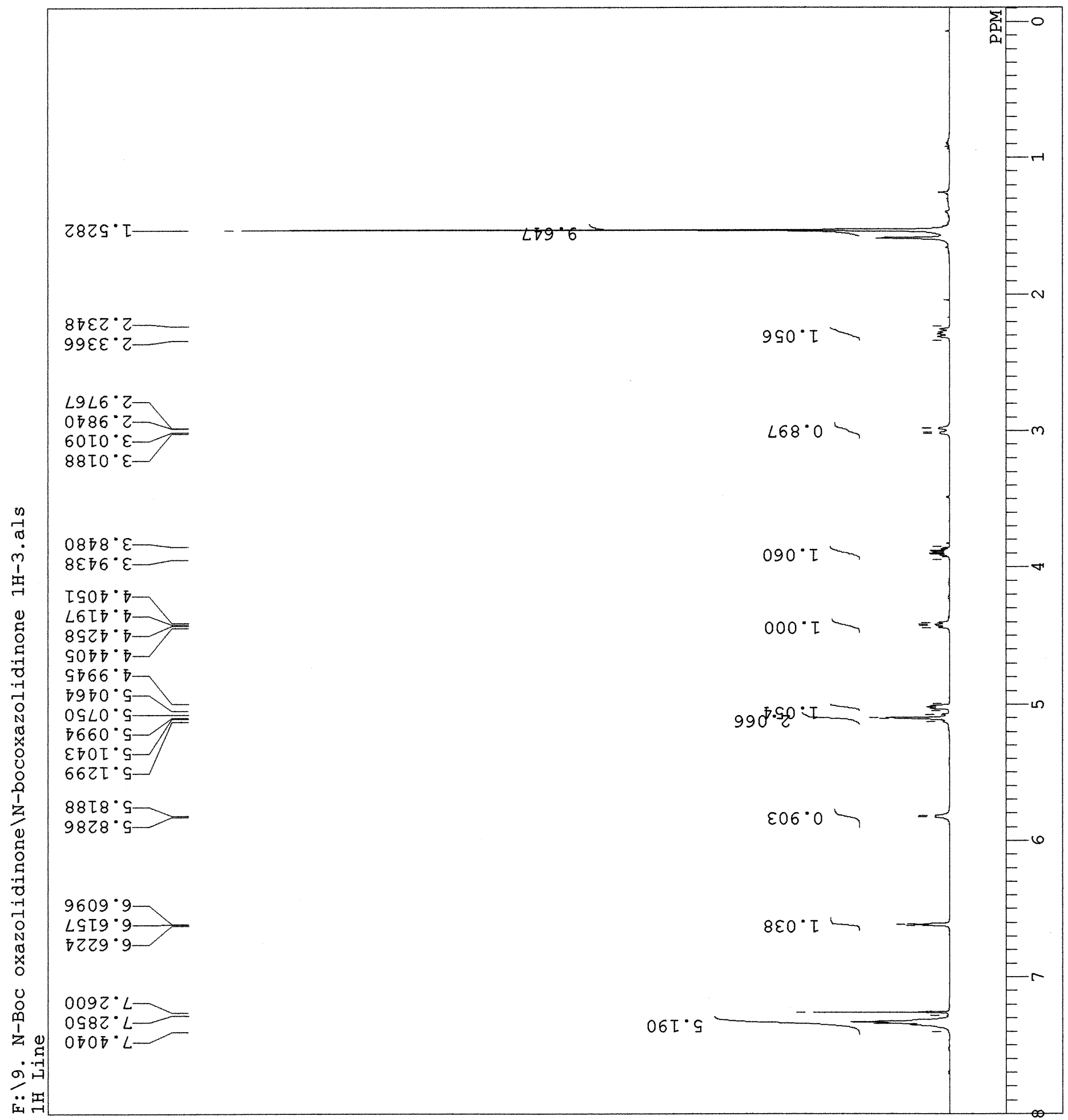


1
1
-1
0
1
0
0
-1
0
1
0
7
0
0
0
0
0

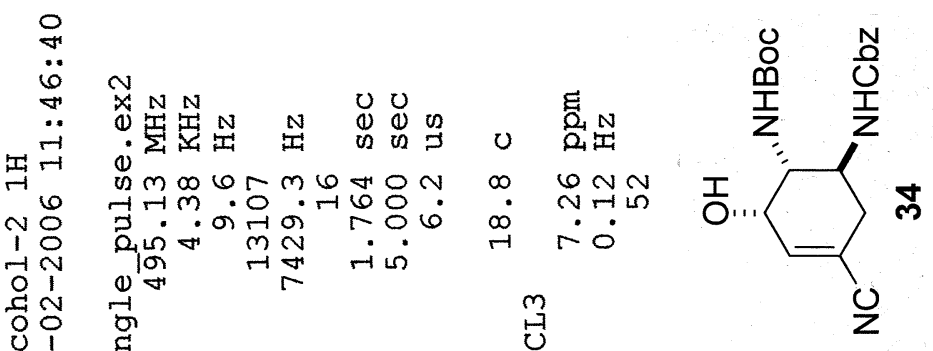

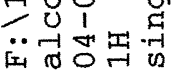

昰 苍

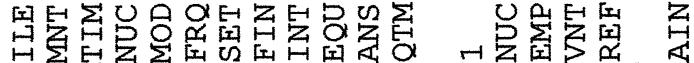

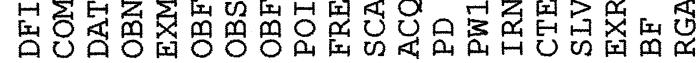

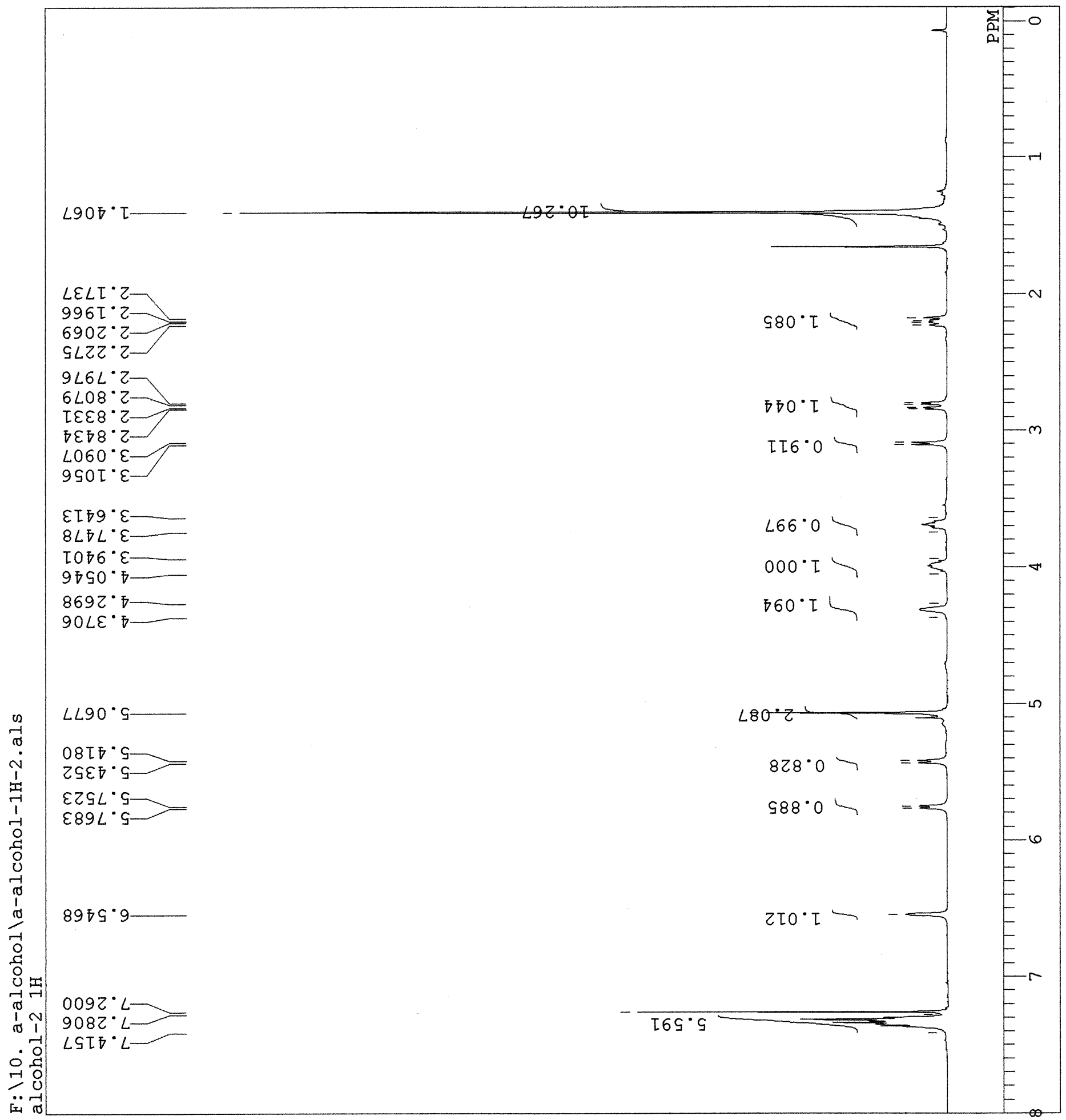


0
0
0
0
1
0
0
0
0
0
0

on

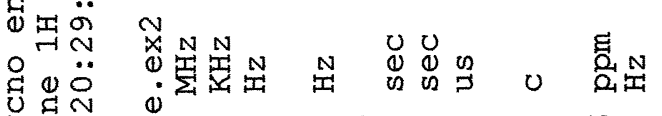

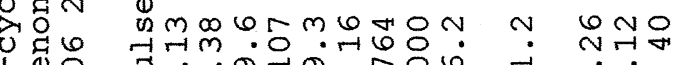

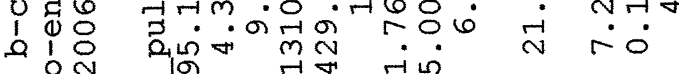

-

걱청 검

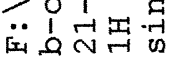

昰苞

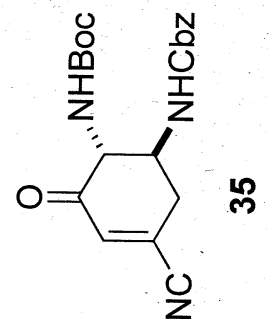

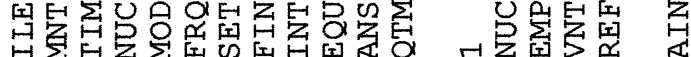

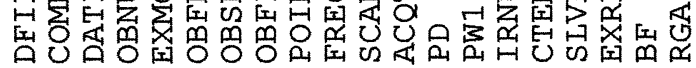

告

$9 \varepsilon \tau \sigma^{\circ} \tau$

$\varepsilon 9 \varepsilon 0^{\circ} \cdot \tau$
$2829 \cdot \tau$

$6 \varepsilon \varepsilon 9^{\circ} 乙$

$88 \pi 9^{\circ} Z$

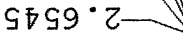

$8799 \cdot z-$

$90 \angle 9^{\circ} z$

ธऽ89. Z-

$\varepsilon 269^{\circ} z$

$6660^{\circ} \varepsilon-$

$060 \tau^{\circ} \varepsilon-$

$\varsigma 9 \varepsilon \tau \cdot \varepsilon-$

9ऽธโ $\varepsilon$

$\angle 896^{\circ} \varepsilon$

$9980^{\circ} \overline{7}$

$887 \varepsilon^{\circ} \nabla$

$9990^{\circ}$

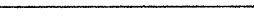

$2 \angle 80^{\circ} \mathrm{G}$

$0680^{\circ}$
$9209^{\circ}$

$000^{\circ} I$

$600^{\circ} \mathrm{I}$

L乙EE' 9

ZLZ9.9

$6929 \cdot 9$

영 छ

U⿺辶一

$0092^{\circ} L$

$8 T 8 Z^{\circ} L$

- 0 哯

OOIE $L$

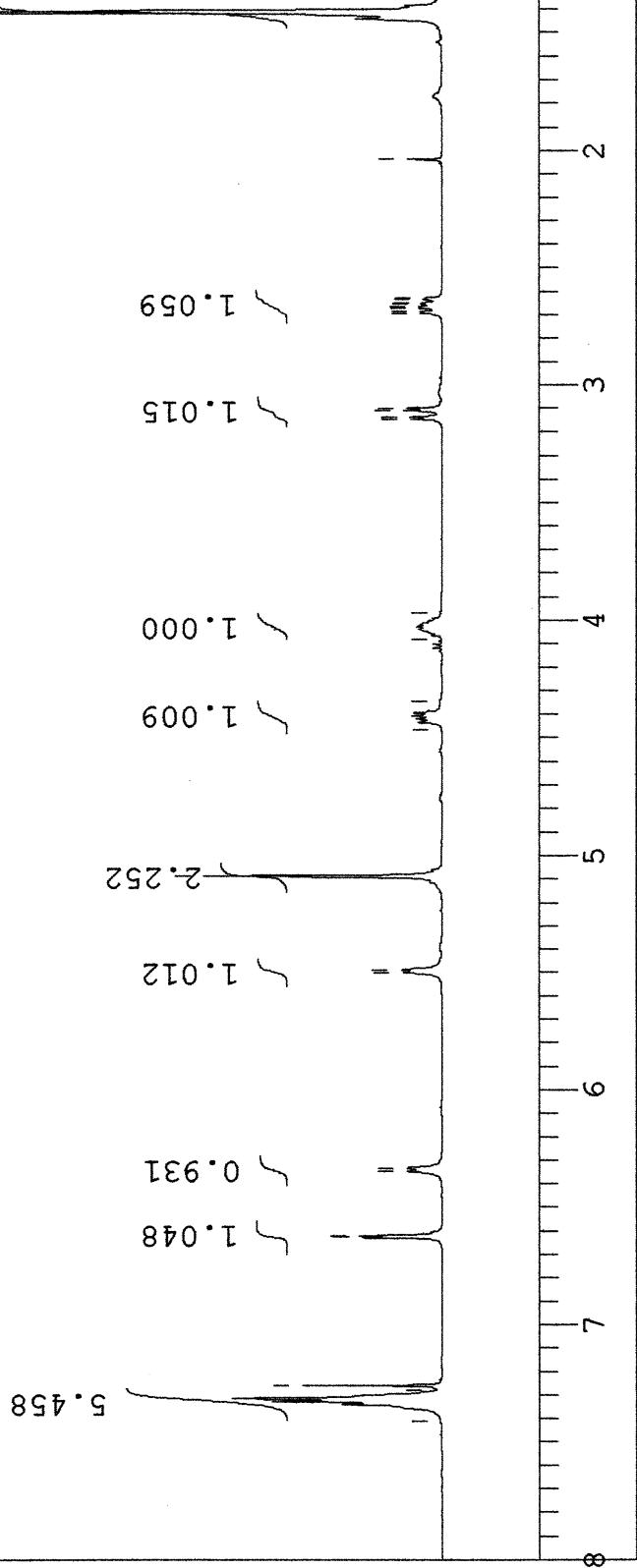




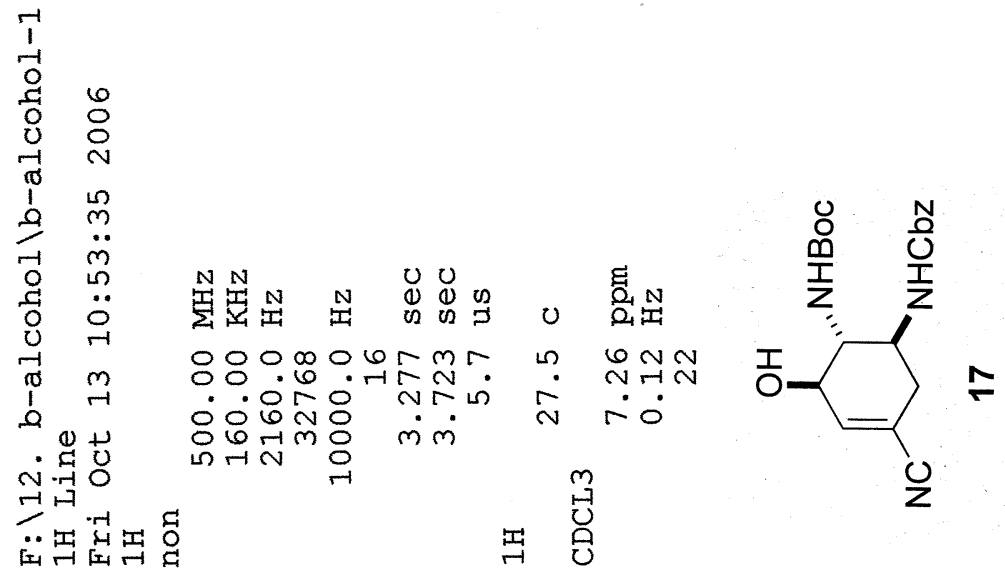

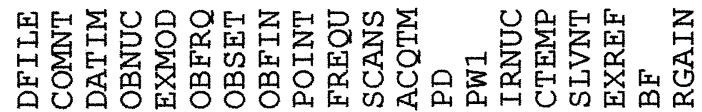

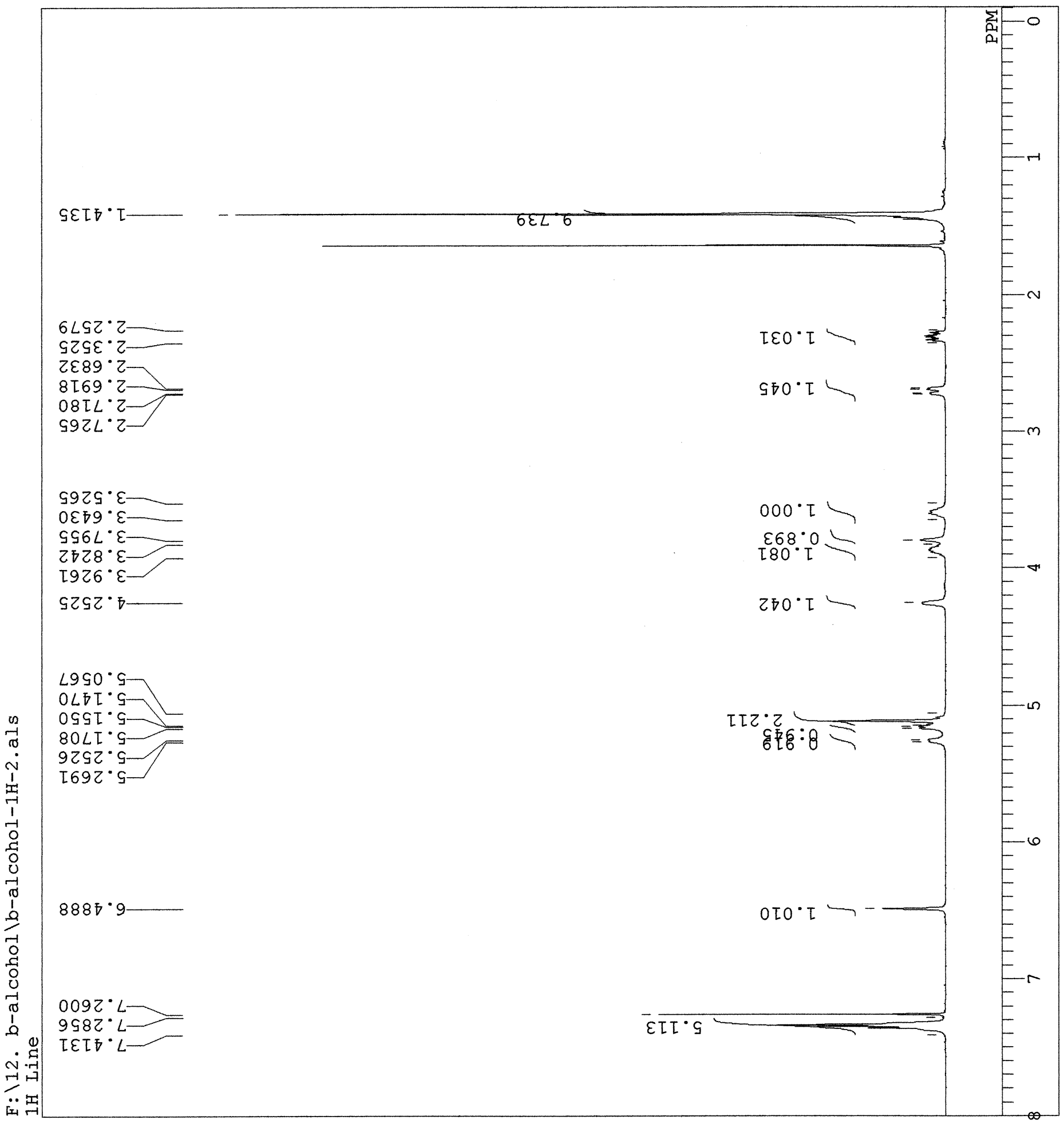




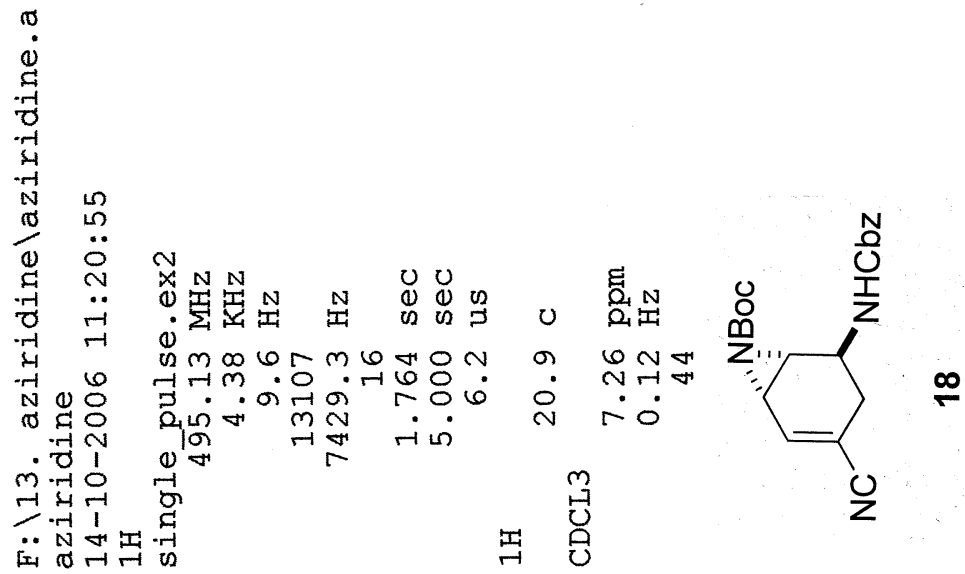

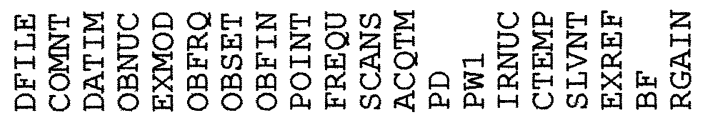

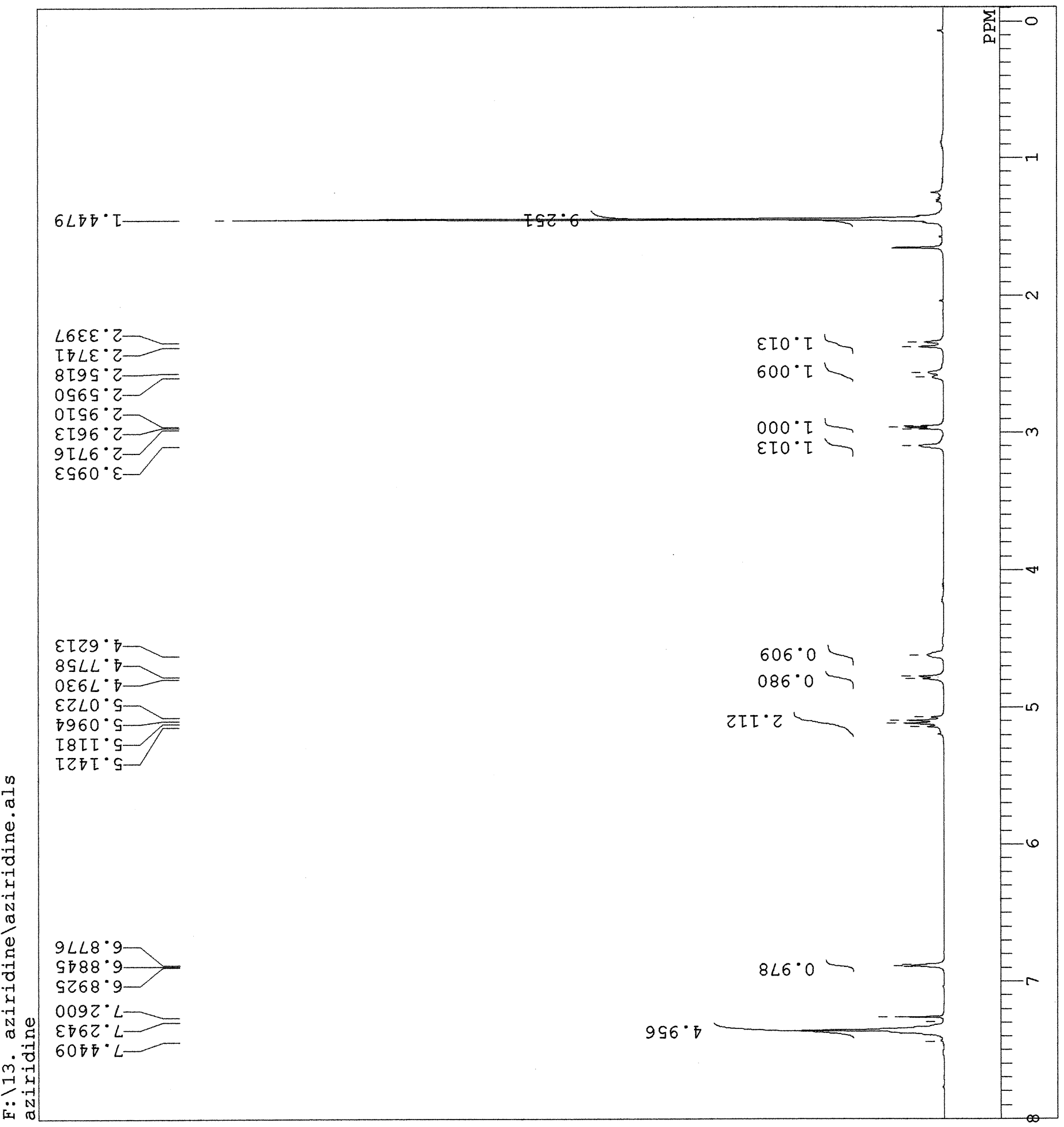


함

$\stackrel{\infty}{\circ}$

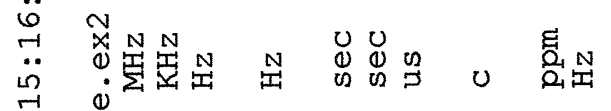

4

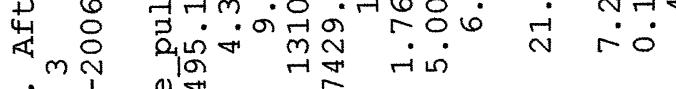

म다 $\begin{gathered}0 \\ 0\end{gathered}$

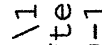

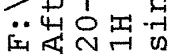

浆苞

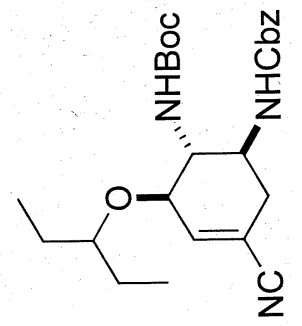

m

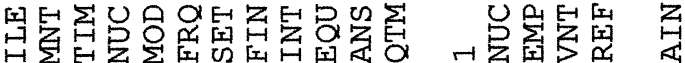

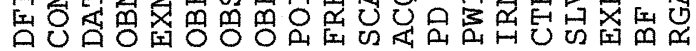

OLL8 ${ }^{\circ}$

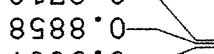

$\angle 006^{\circ} 0$

ZTLE・T-

9ES ${ }^{\circ}$

$S \angle D S \cdot[-$

$90 L \varepsilon^{\circ} Z$

$8 \angle 8 \varepsilon^{\circ} Z$

โ907.Z

ยह乙๐ 乙

Oโโ9. 2

ฤ૬ธ9・て

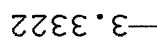

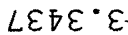

โSSE $\varepsilon^{\circ} \varepsilon$

$9 \bar{L} L^{\circ} \varepsilon$

$8 โ \varepsilon L \cdot \varepsilon$

$68 \nabla L \cdot \varepsilon$

I99L $L^{\circ} \varepsilon$

0 โ $6^{\circ} \varepsilon$

$\varepsilon 629^{\circ}$

乙๐ $59^{\circ} \square$

$26 \mathrm{LO} 0^{\circ} \mathrm{-}$

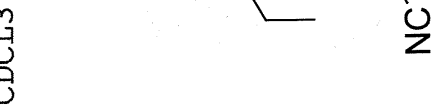

.

\section{(n)}




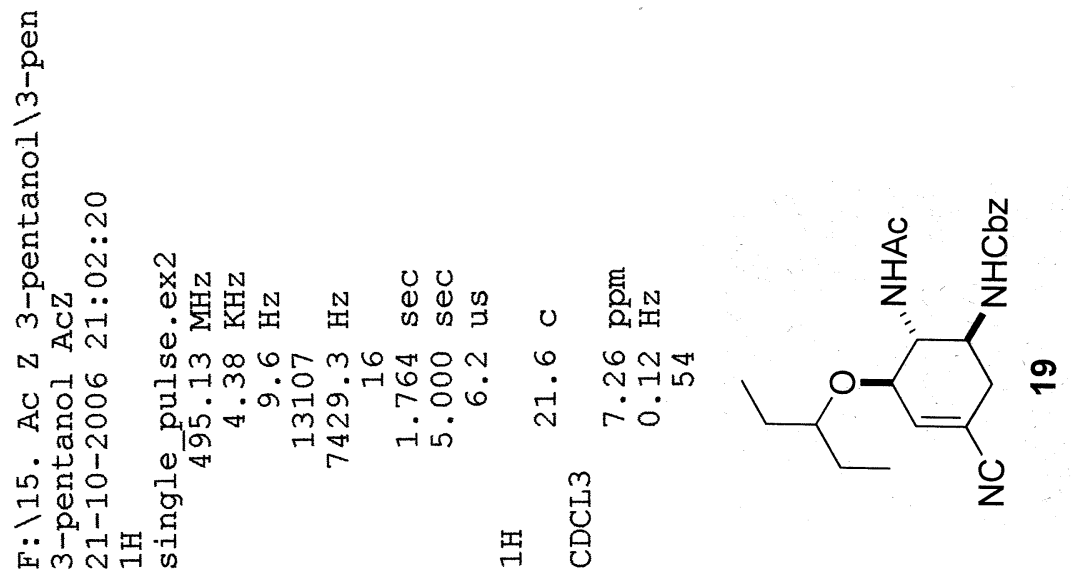

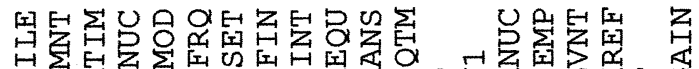

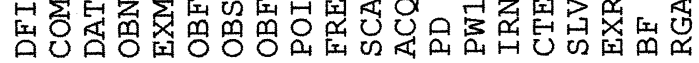

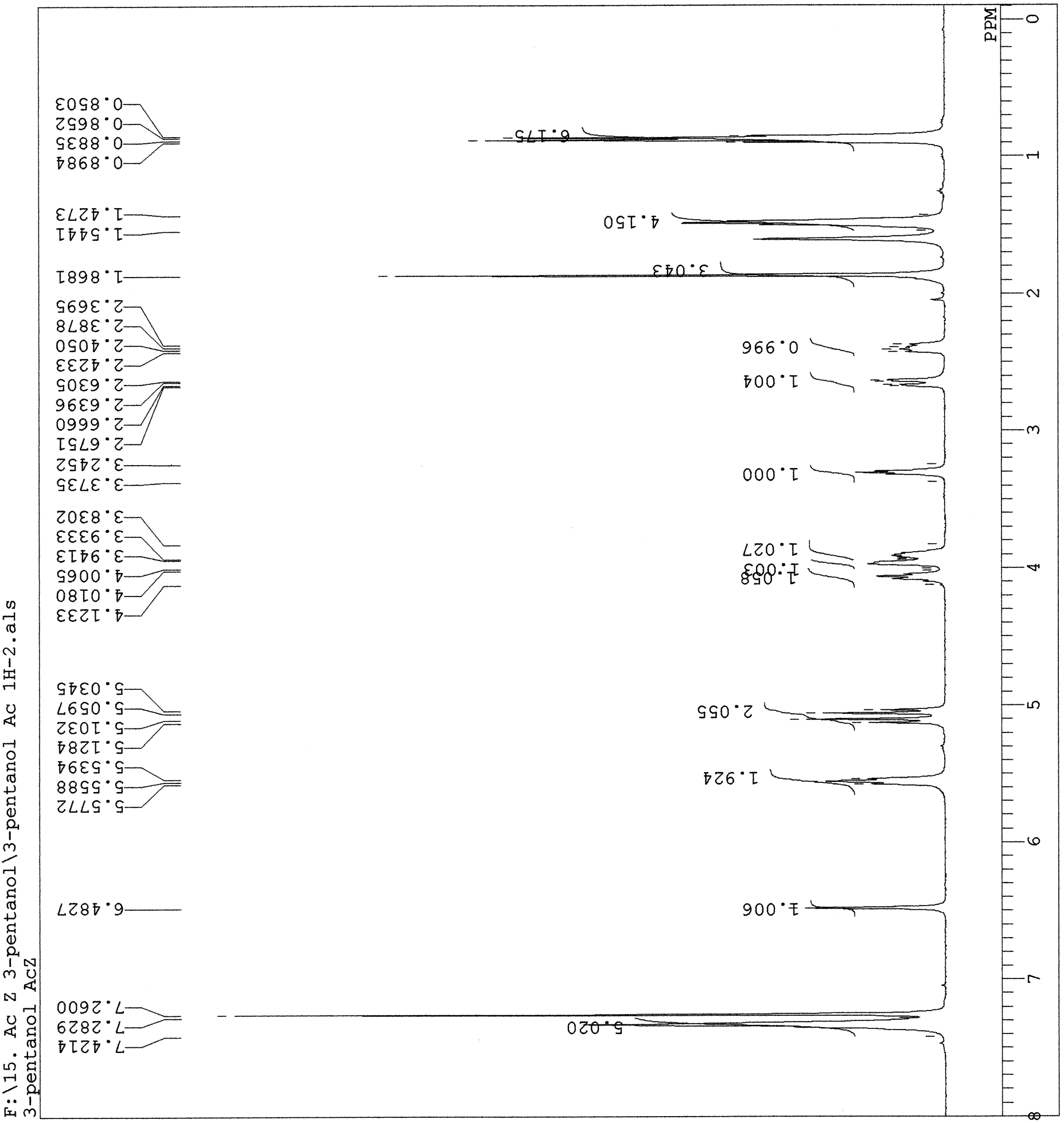




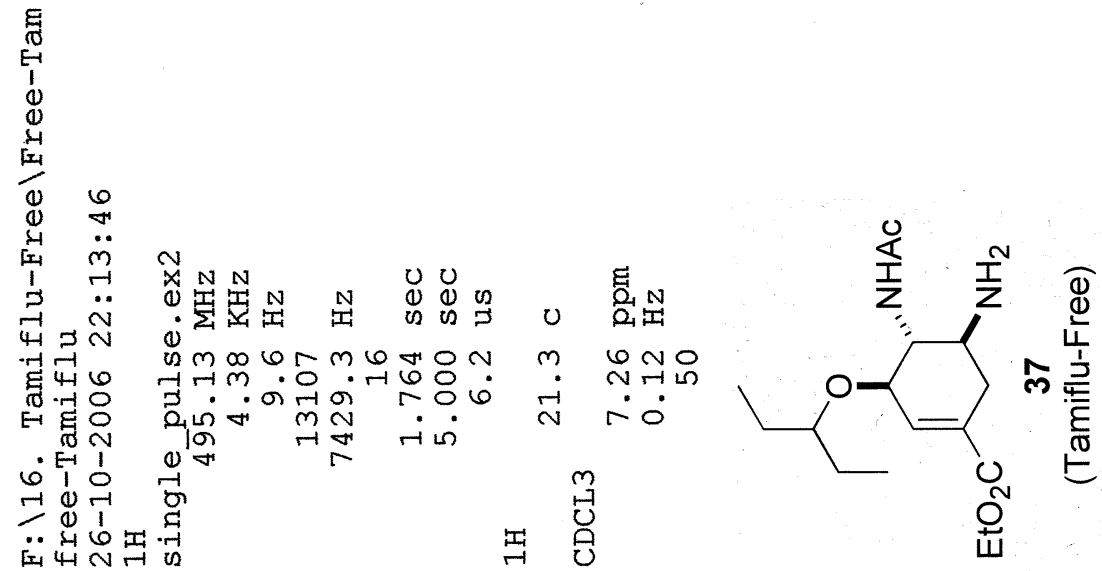

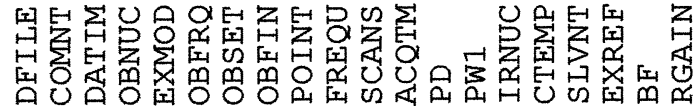

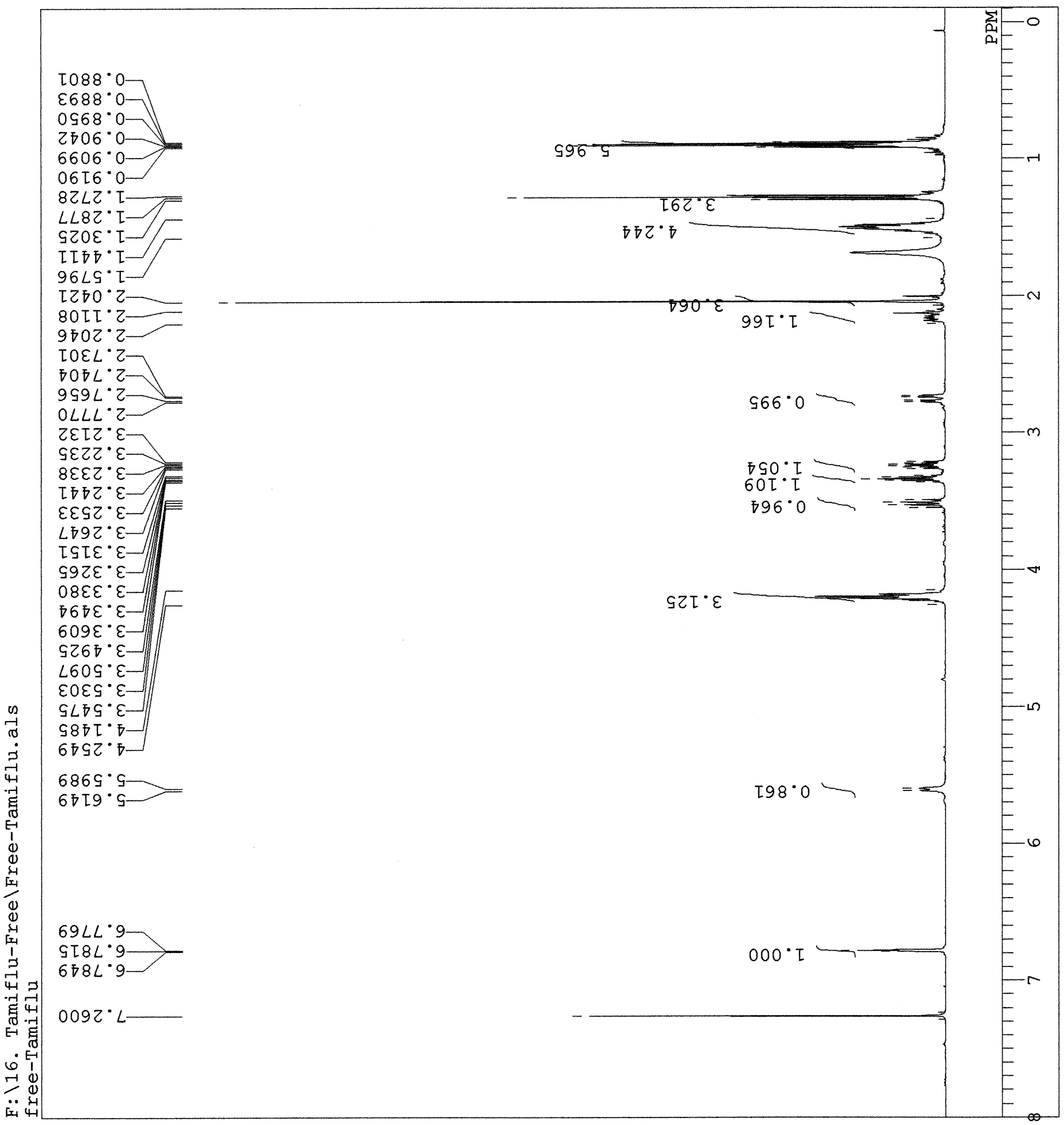




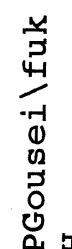

-1
0

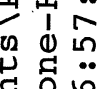

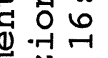

구

덩응

西

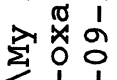

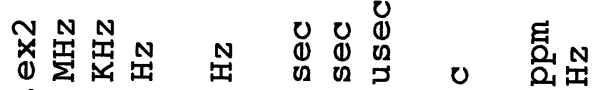

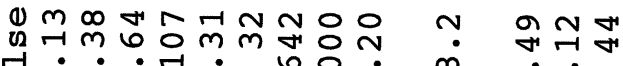

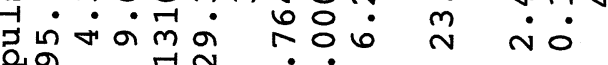

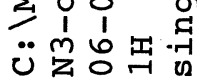

出 筫

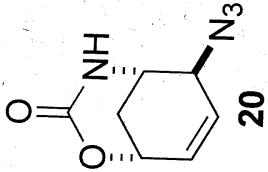

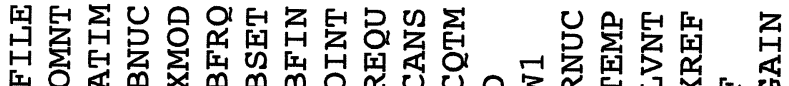

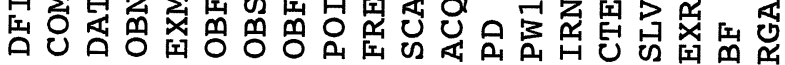

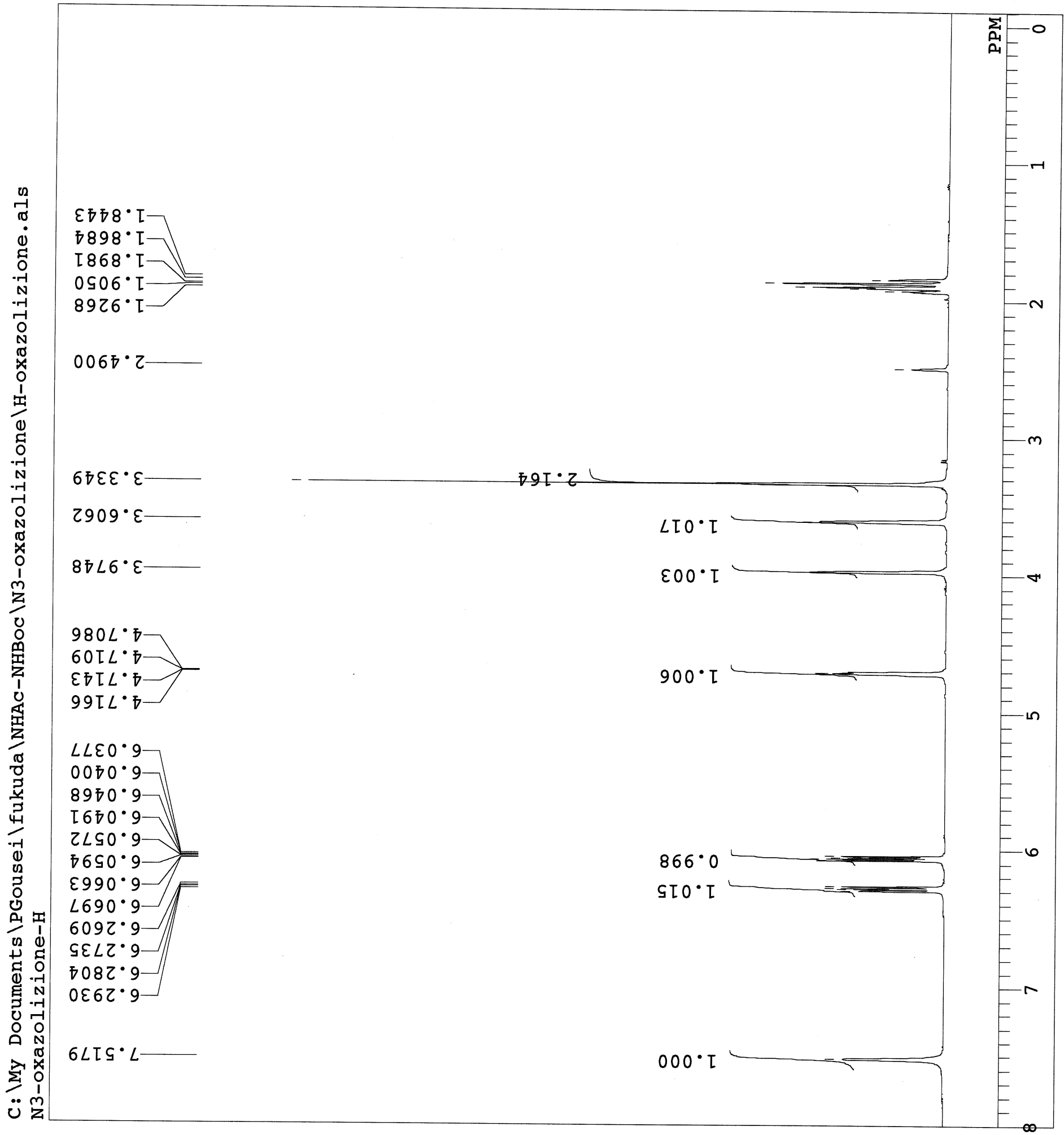


ये

$\begin{array}{lll}0 & 0 \\ 0 & 0 \\ 0 & 0 \\ 1 & 0 \\ z & 1\end{array}$

$\dot{\sim} \stackrel{\sim}{N}$

$\stackrel{m}{\ddot{m}}$

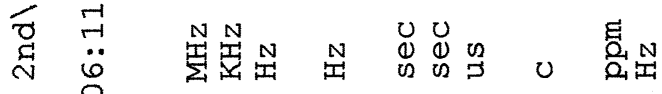

T OOODOGMRN m GNN

- 4 r.

出

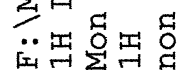

㒴苞

$0=\sum_{0}^{\infty} \sum_{m}^{\infty}$

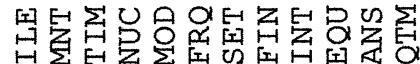

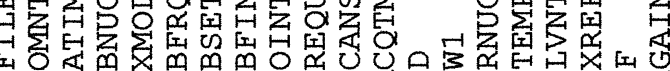

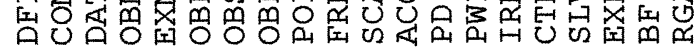

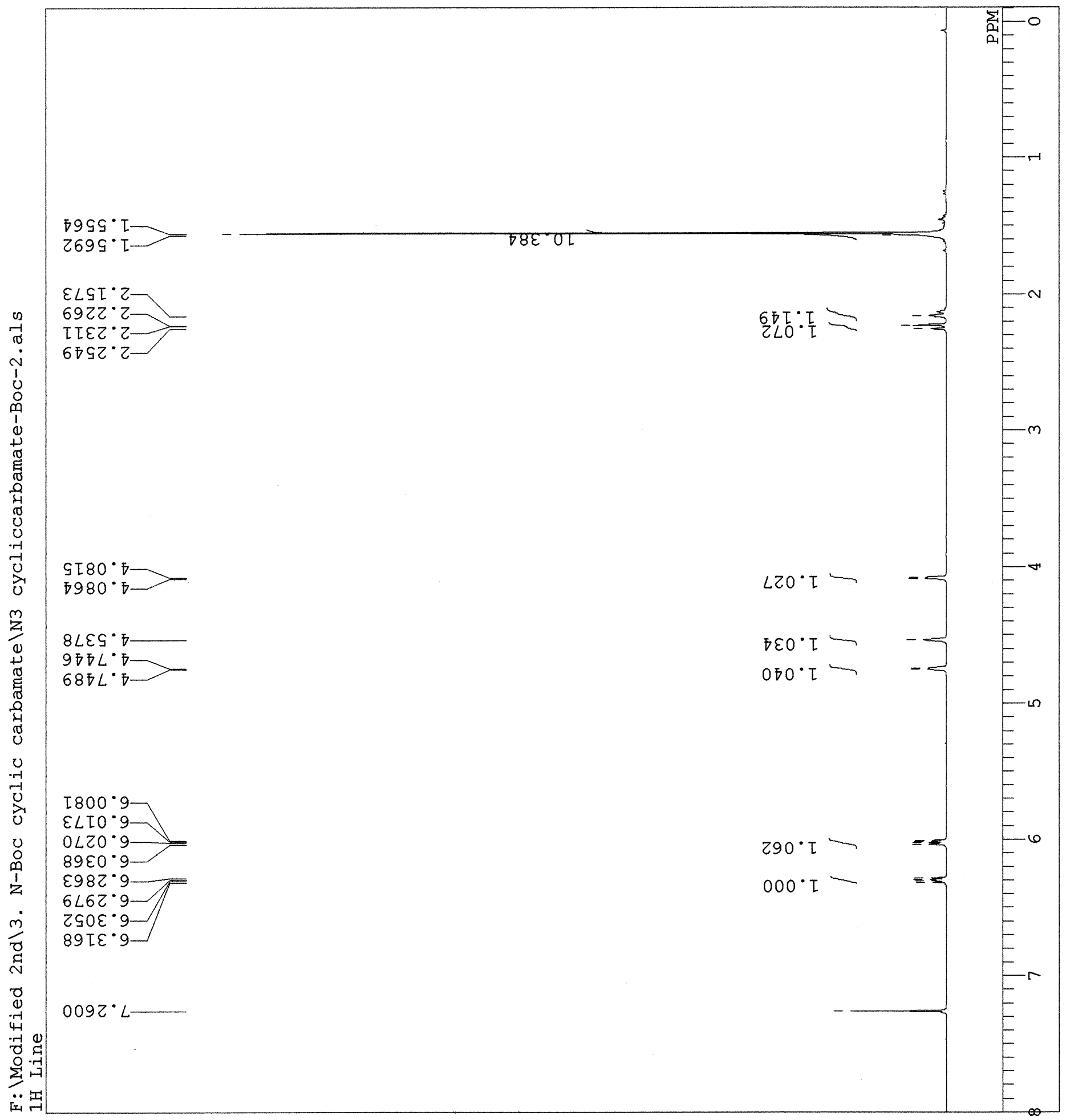


压安

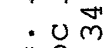

$\forall \circ$

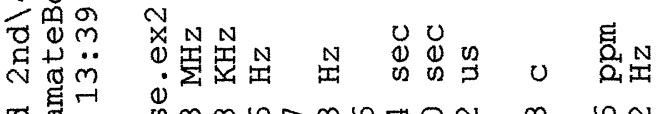

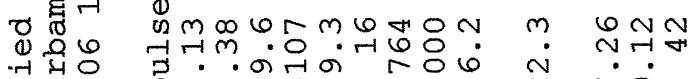

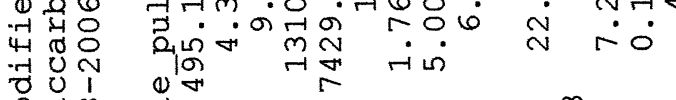

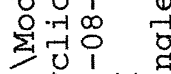

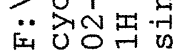

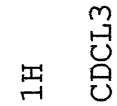

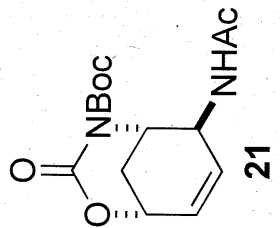

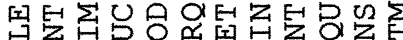

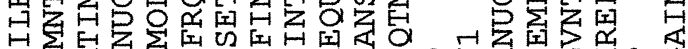

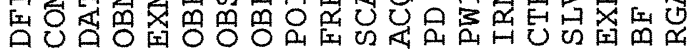

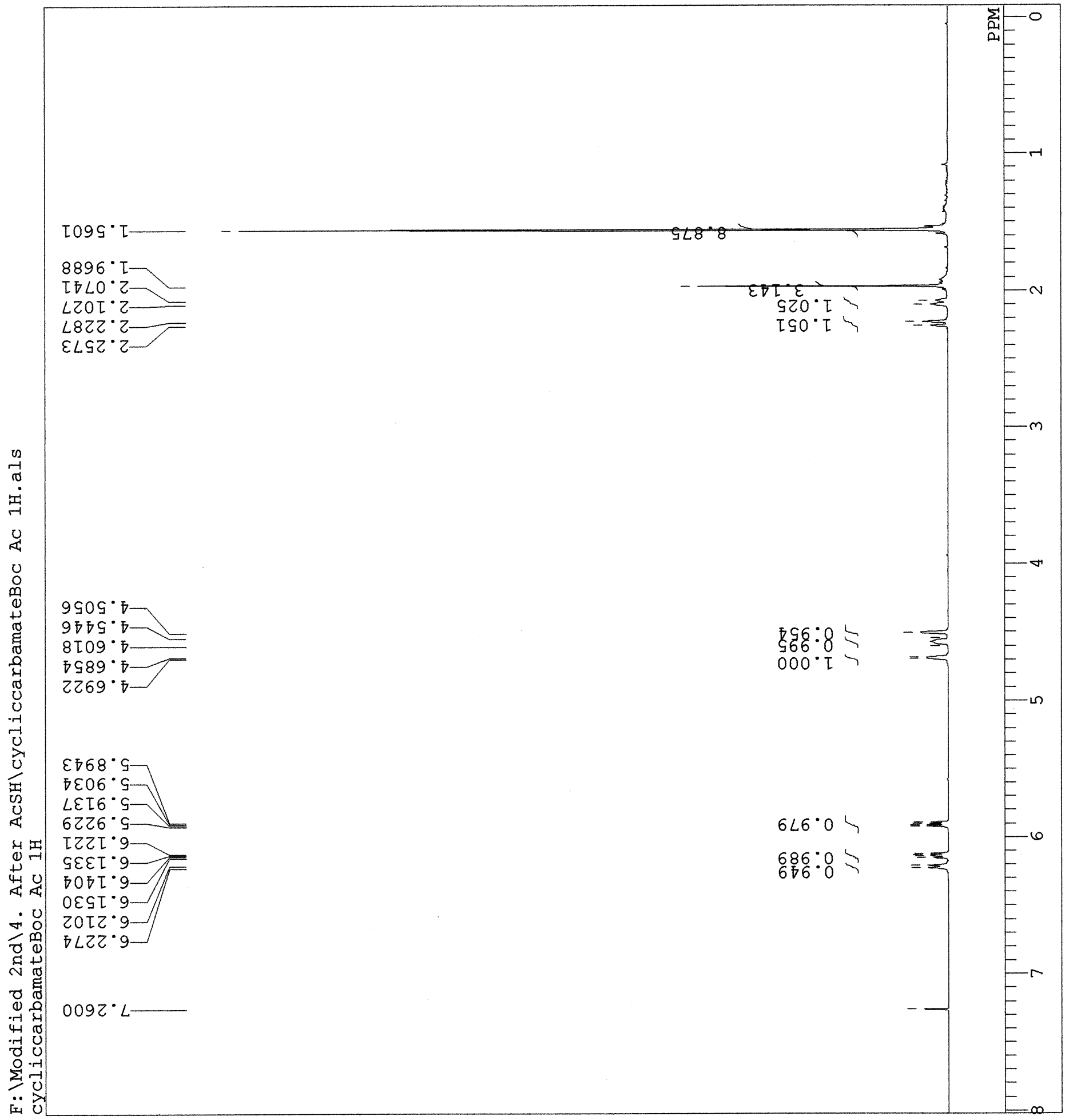


- $\stackrel{+}{N}$

$\frac{10}{2} \ddot{m}$

荧

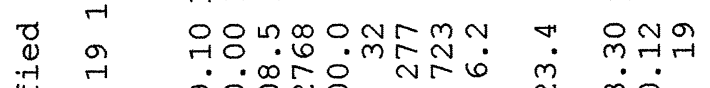

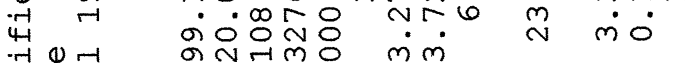

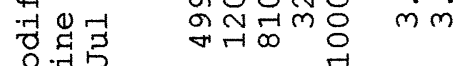

出 $\stackrel{0}{0}$ 웜

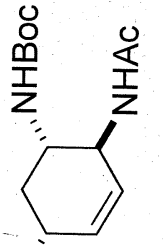

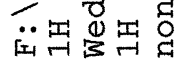

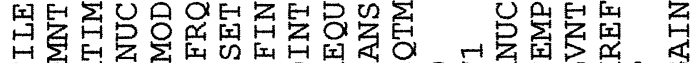

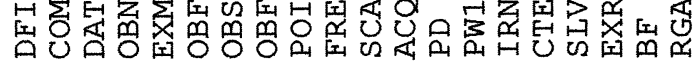

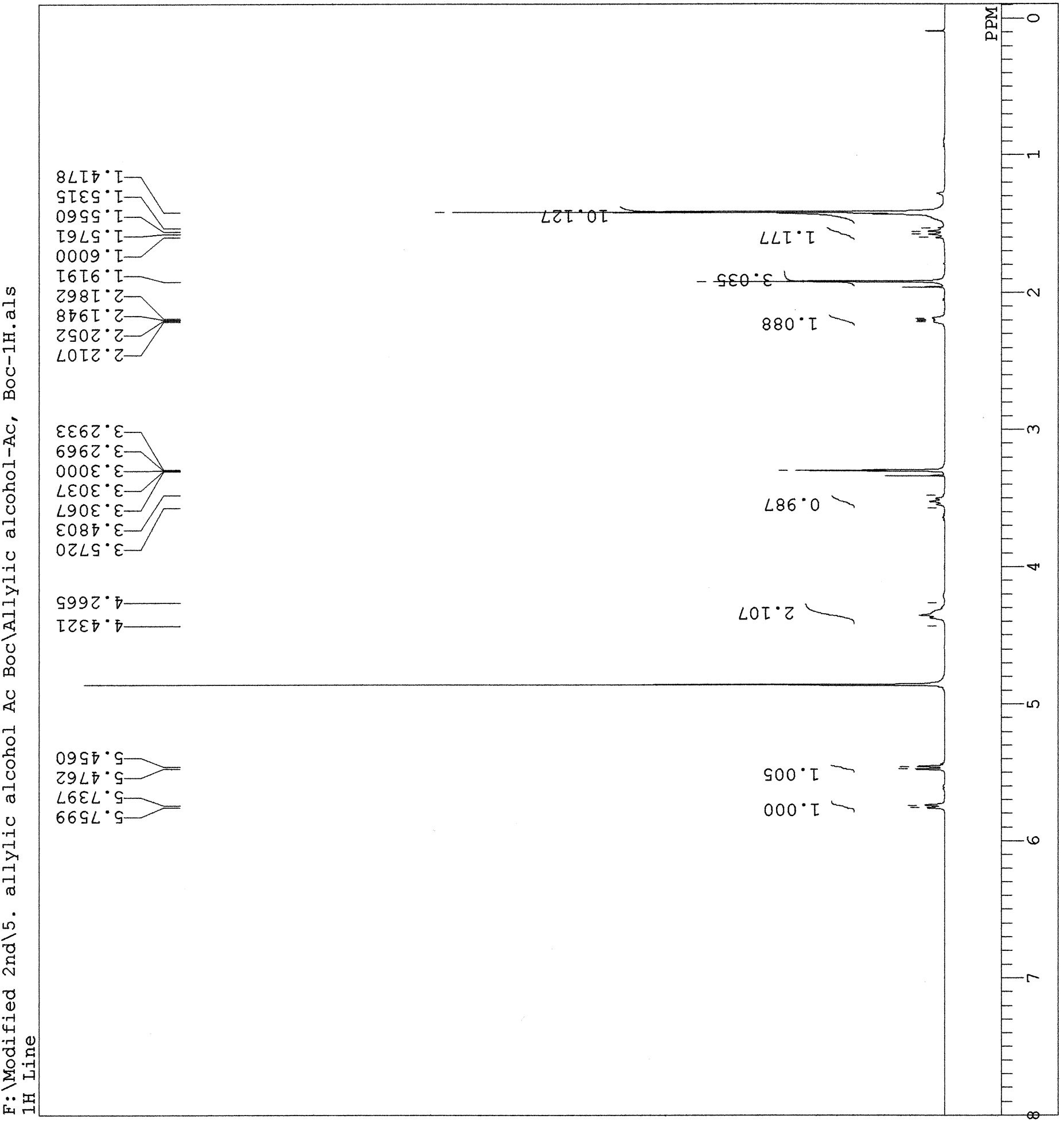


$\begin{array}{ll}\mathbb{1} & 6 \\ 1 & 0 \\ 0 & 0 \\ 0 & N\end{array}$

$\dot{0} \stackrel{-}{\sim}$

$\begin{array}{ll}0 & \ddot{0} \\ \text { v } & \ddot{m}\end{array}$

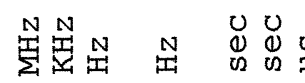

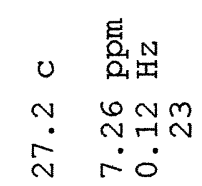

ro 出

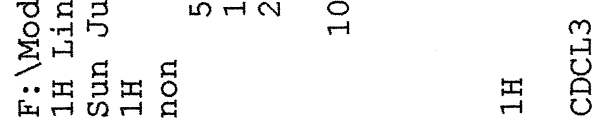

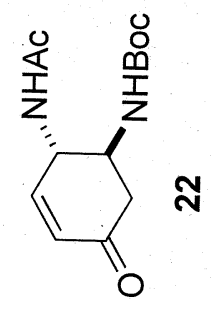

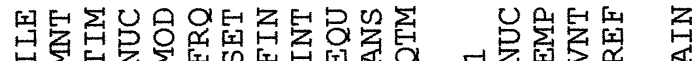

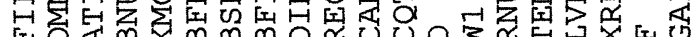

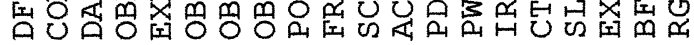

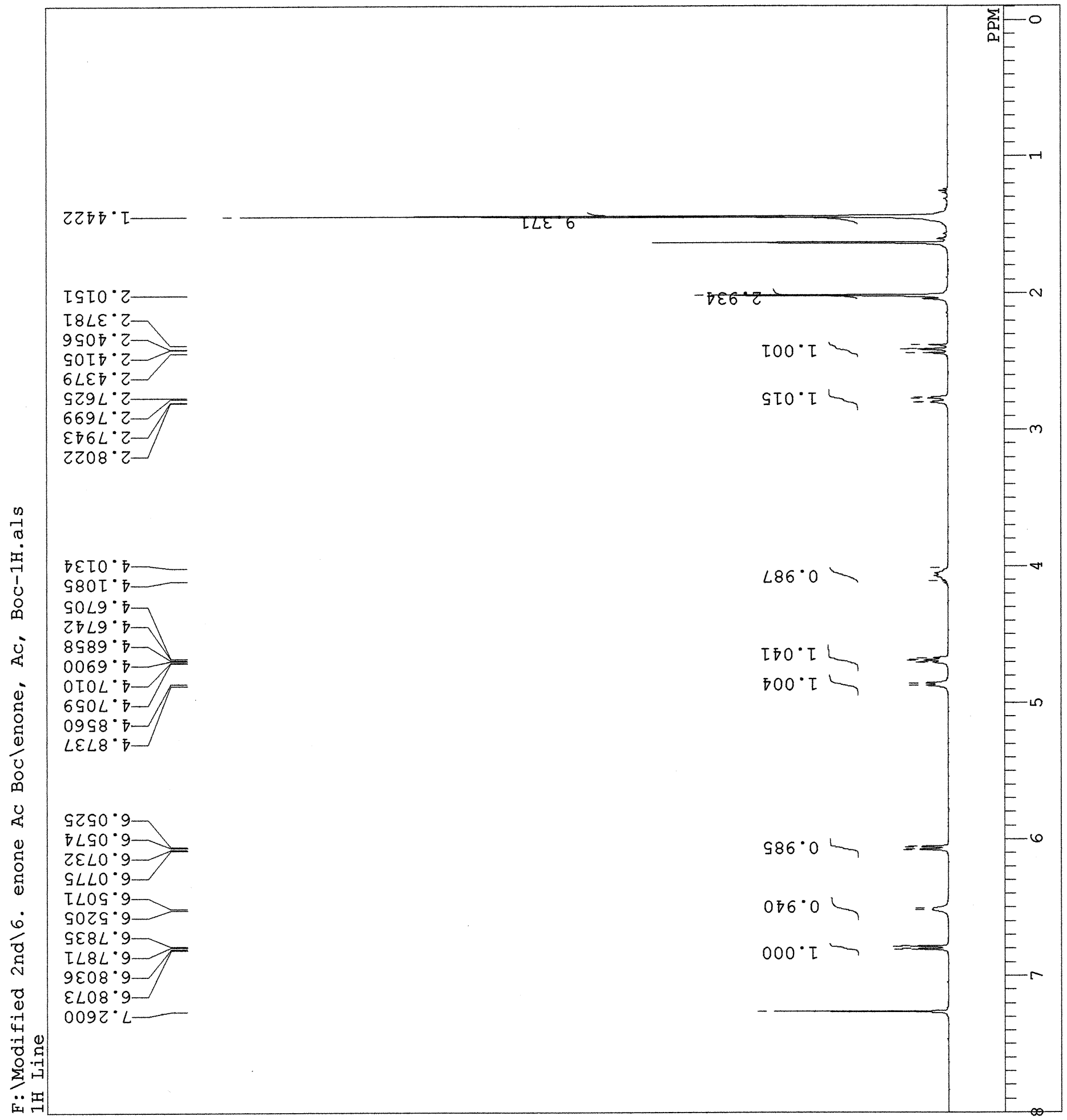




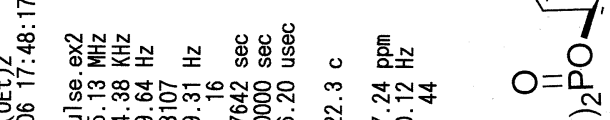

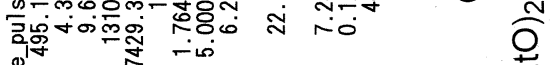

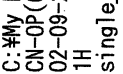
×

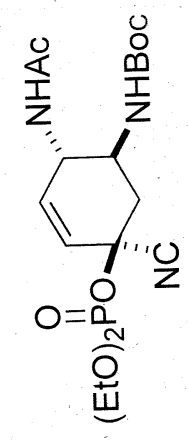

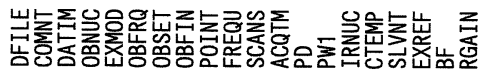

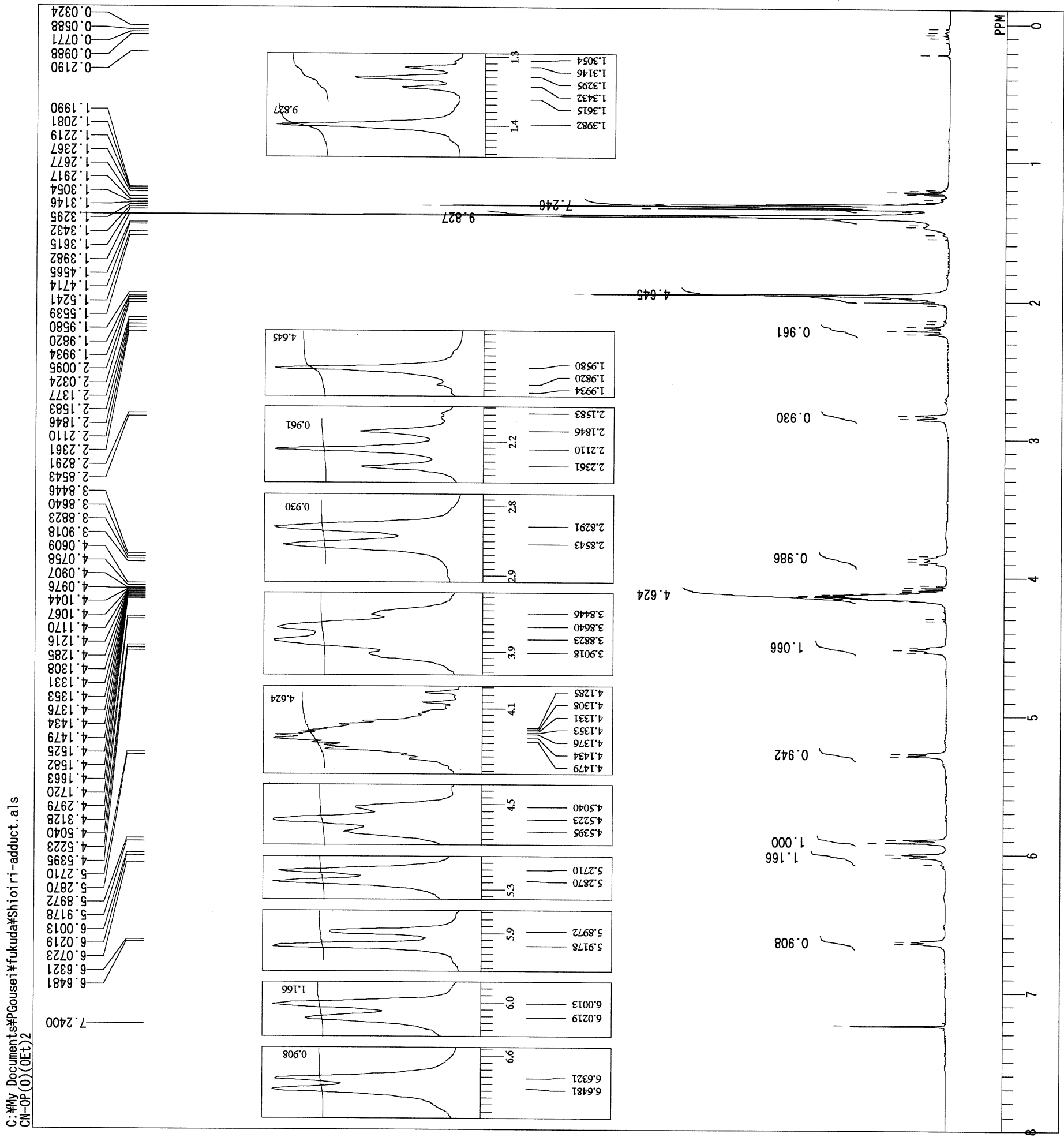




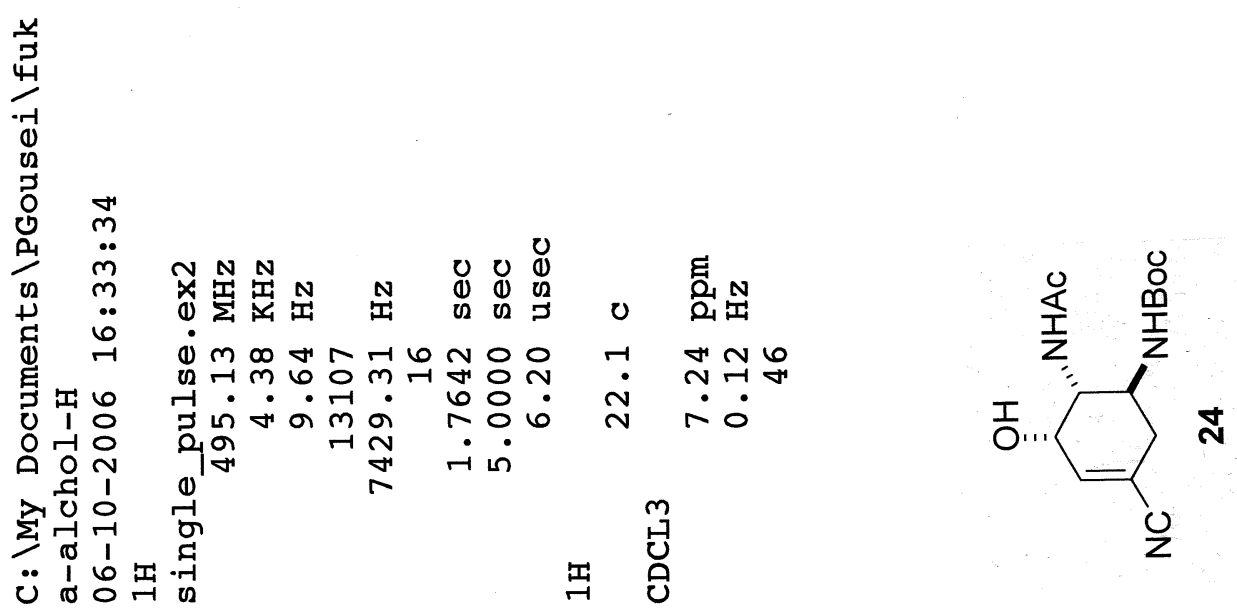

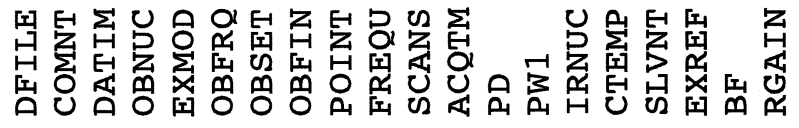

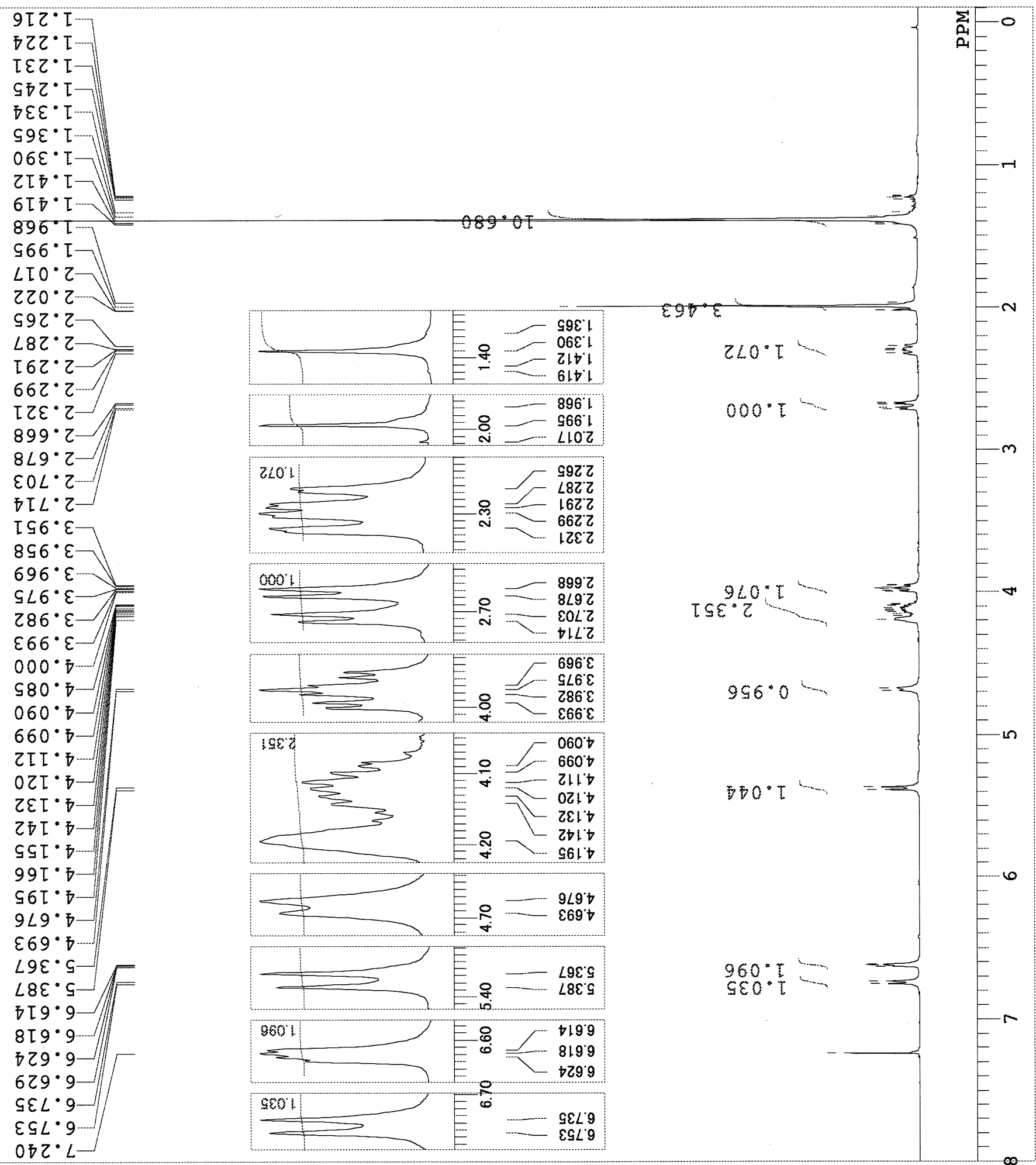




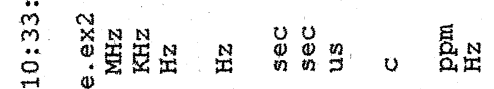

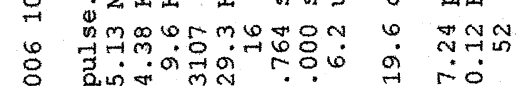

年

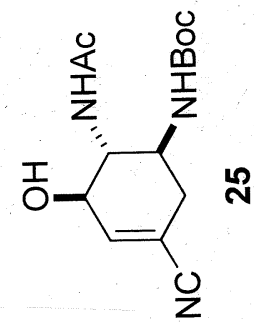

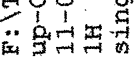

幽葛

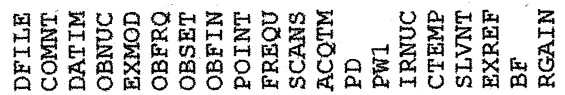

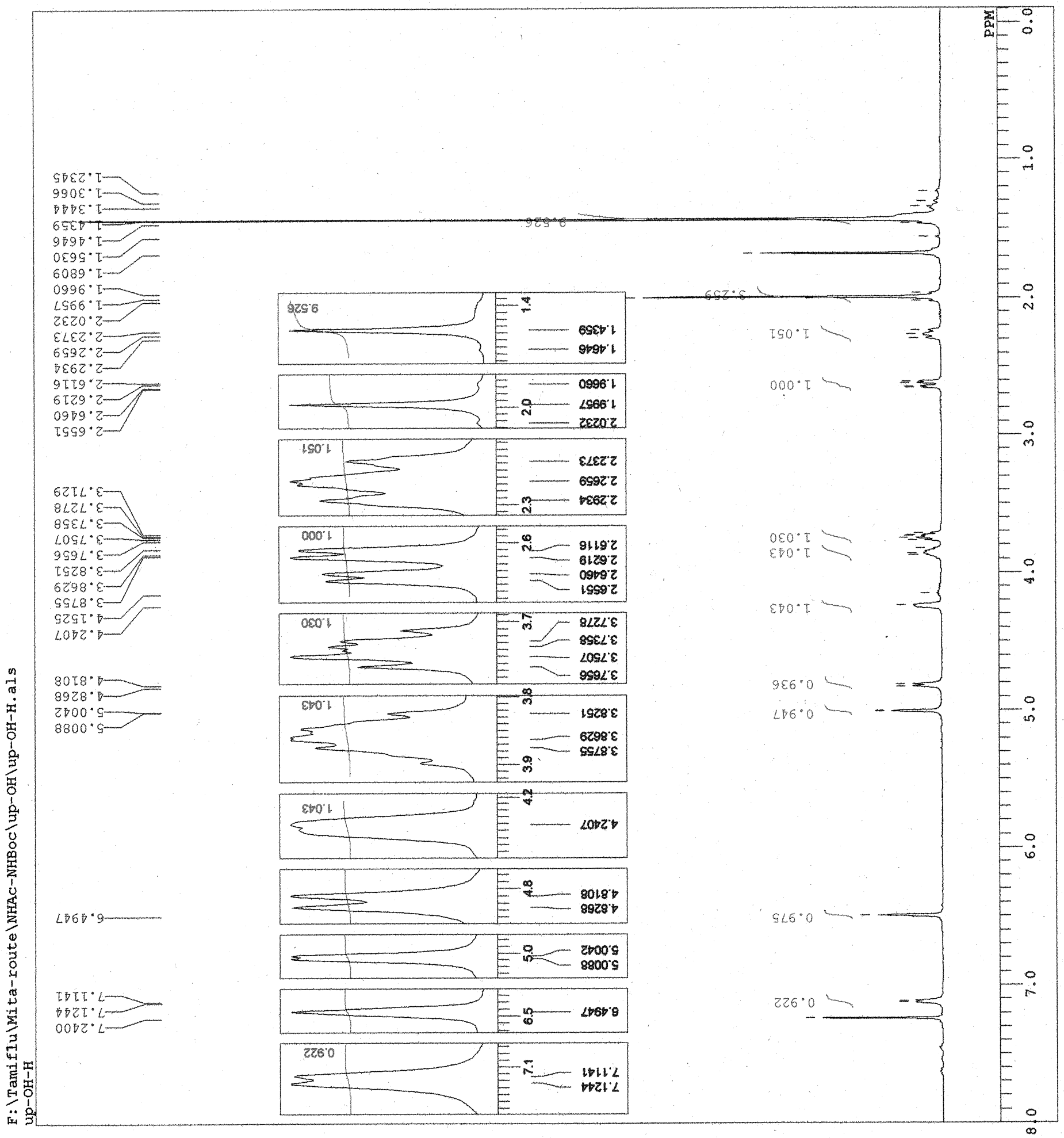




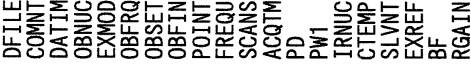

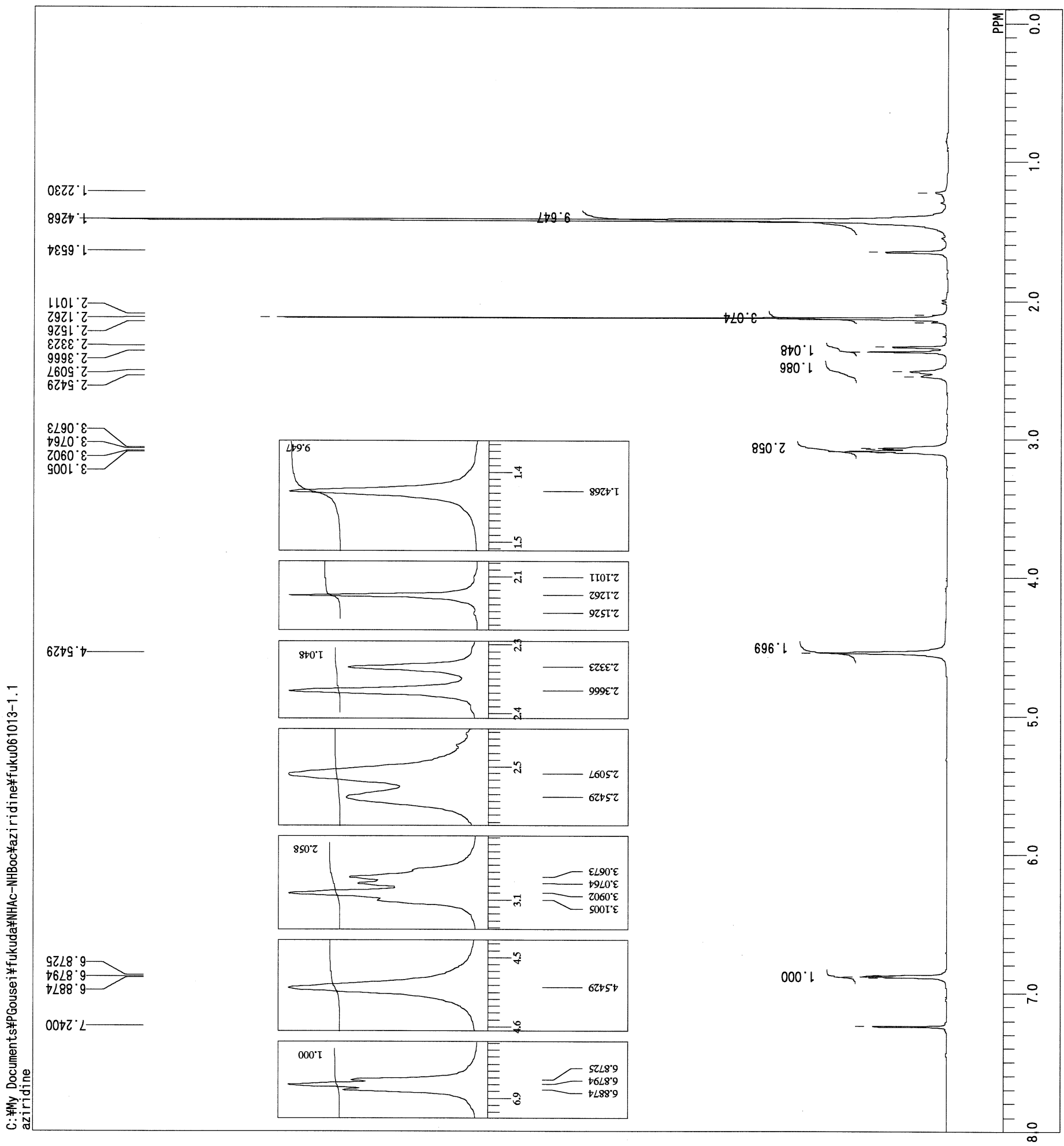




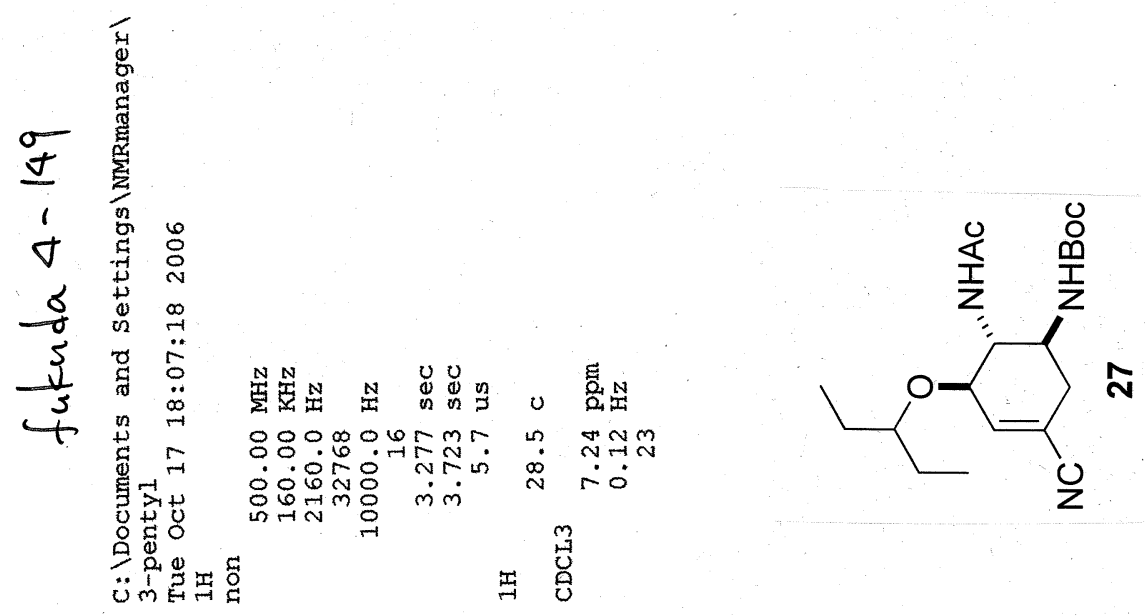

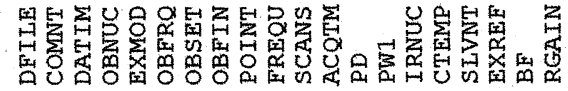

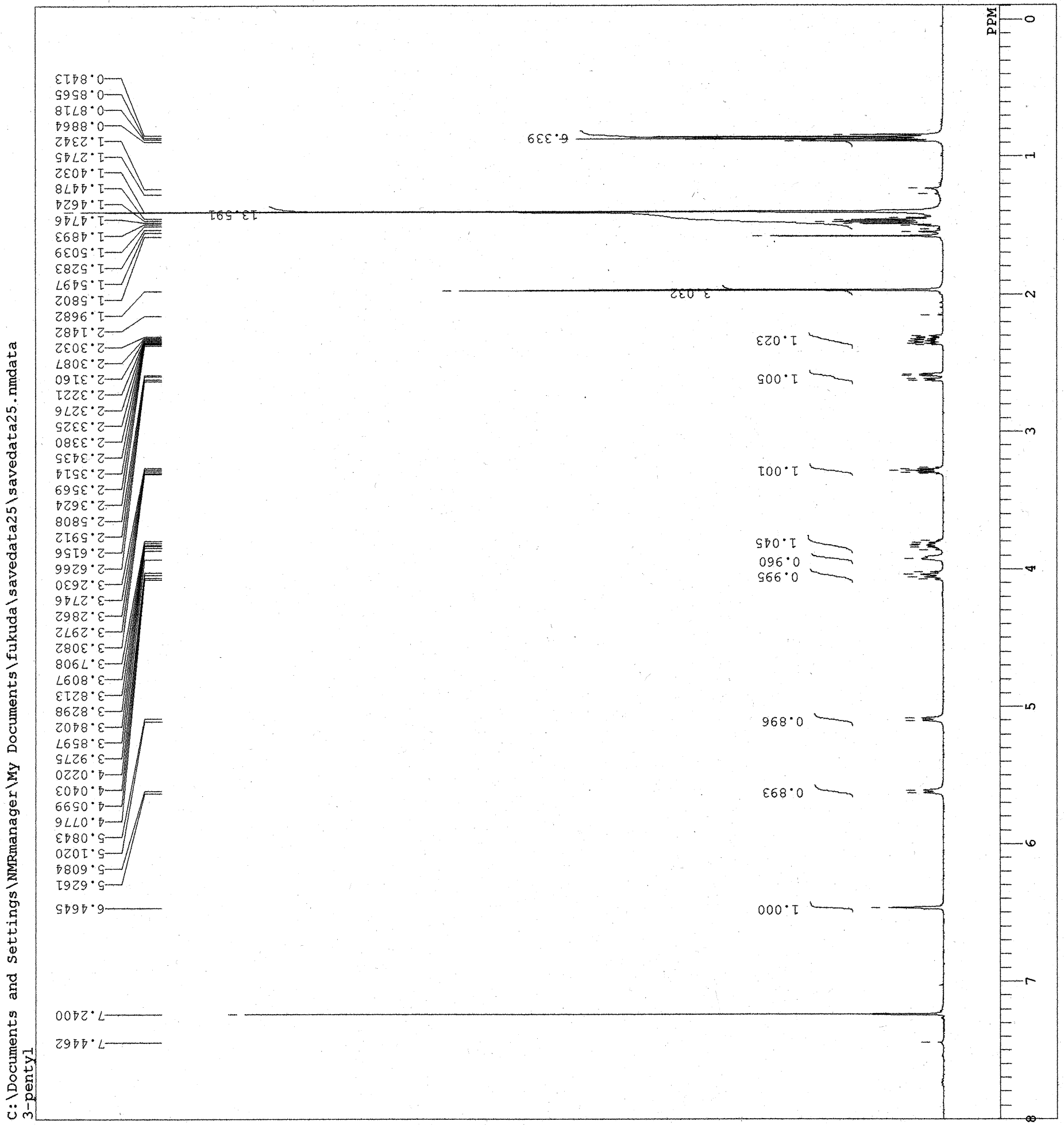




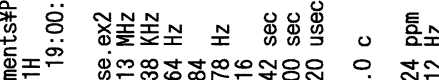

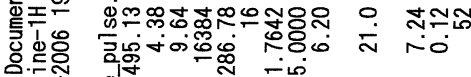

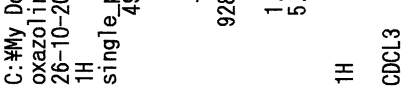

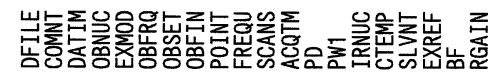
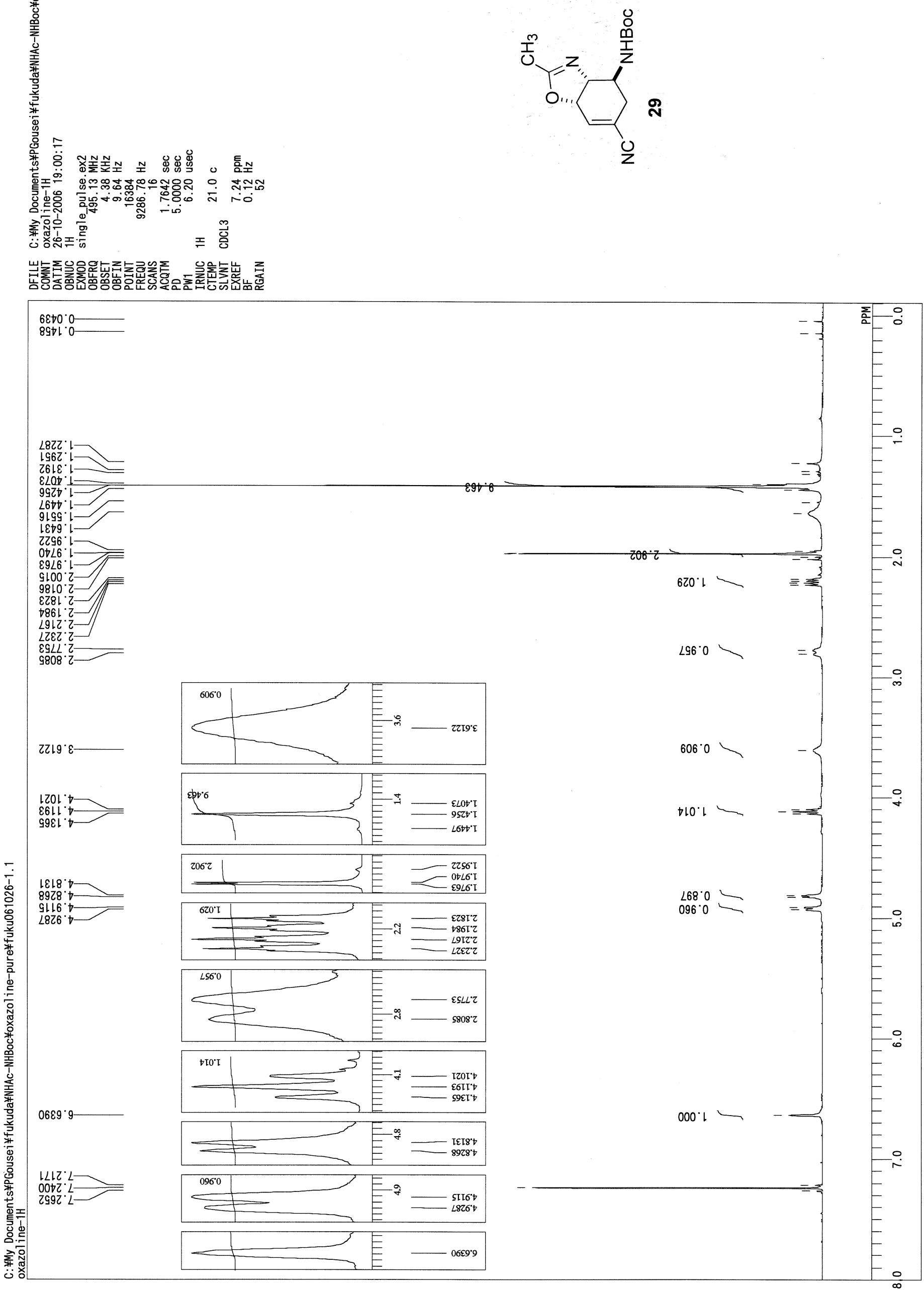


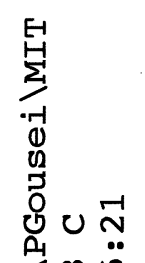

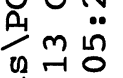

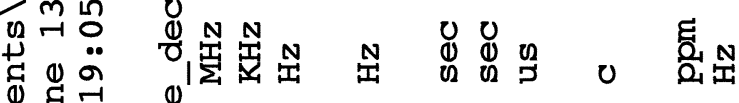

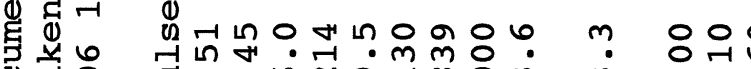

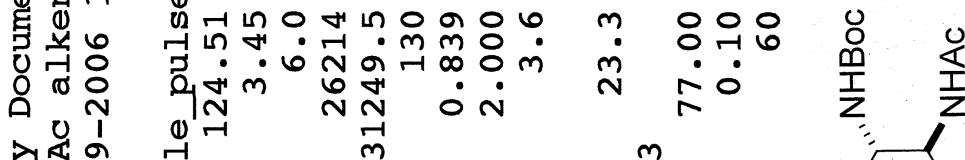

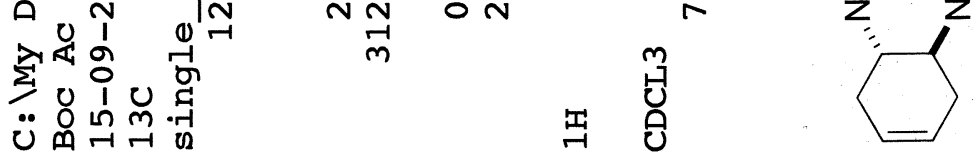

요

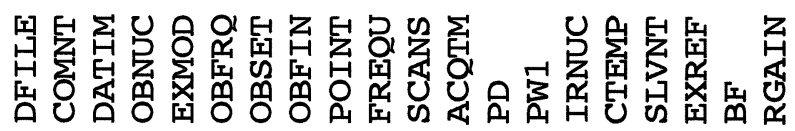

iั

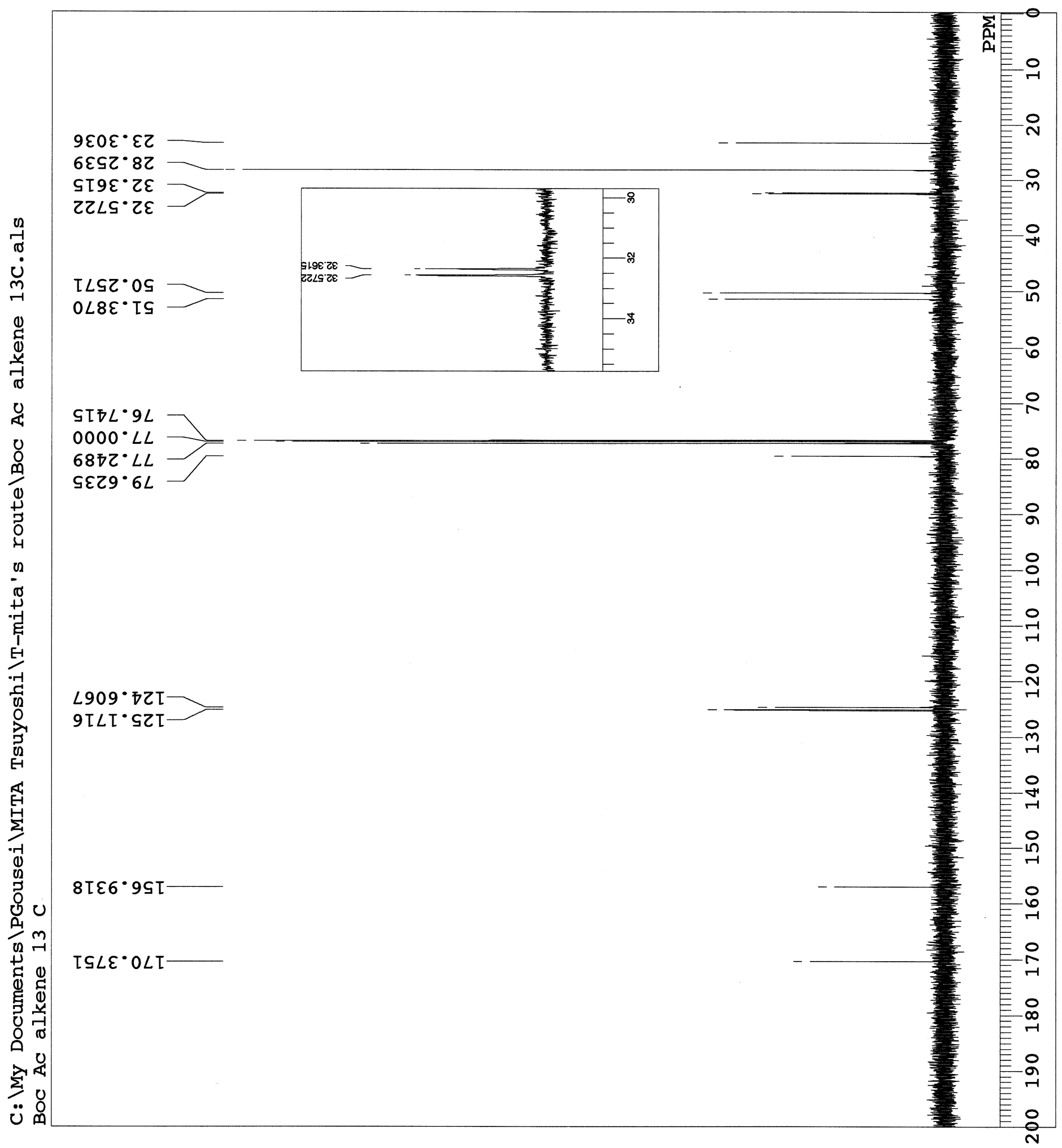




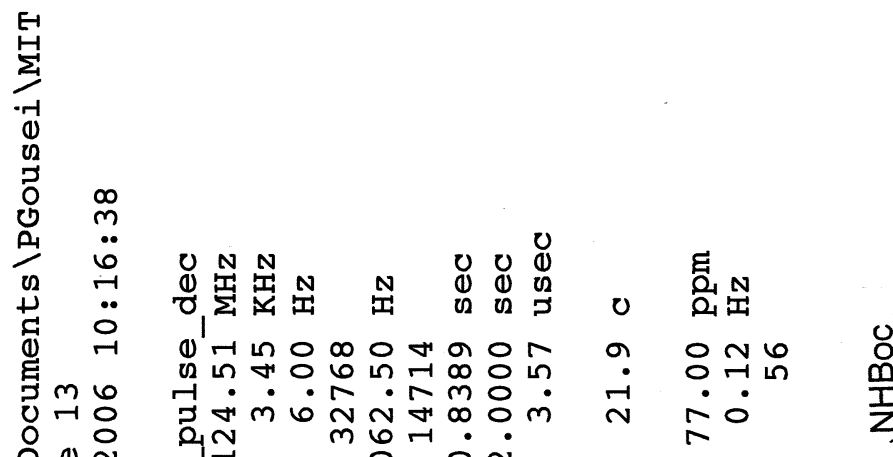

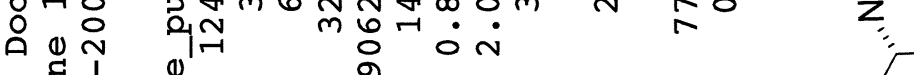

至

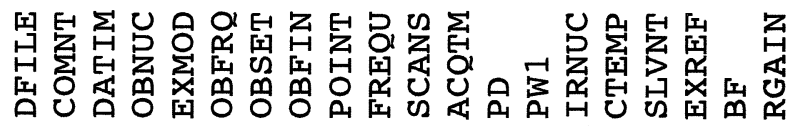

$806 L \cdot \tau Z$

Ђォદ8・て乙

$928 乙 \cdot 82$

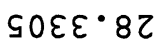
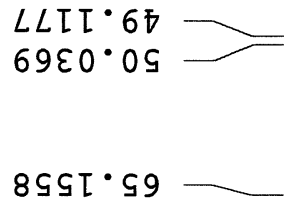

$\angle \varepsilon 00^{\circ} \angle 9$

SI $L \cdot 9 L$

$0000 \cdot L$

$68 \nabla Z \cdot L L$

ह乙ऽ9 $6 L$

$\nabla 9[\varepsilon \cdot L Z I$

OD $\angle 0^{\circ} O \varepsilon L$

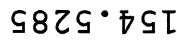

$9 \nabla \nabla 9 \cdot 9 \varsigma T$
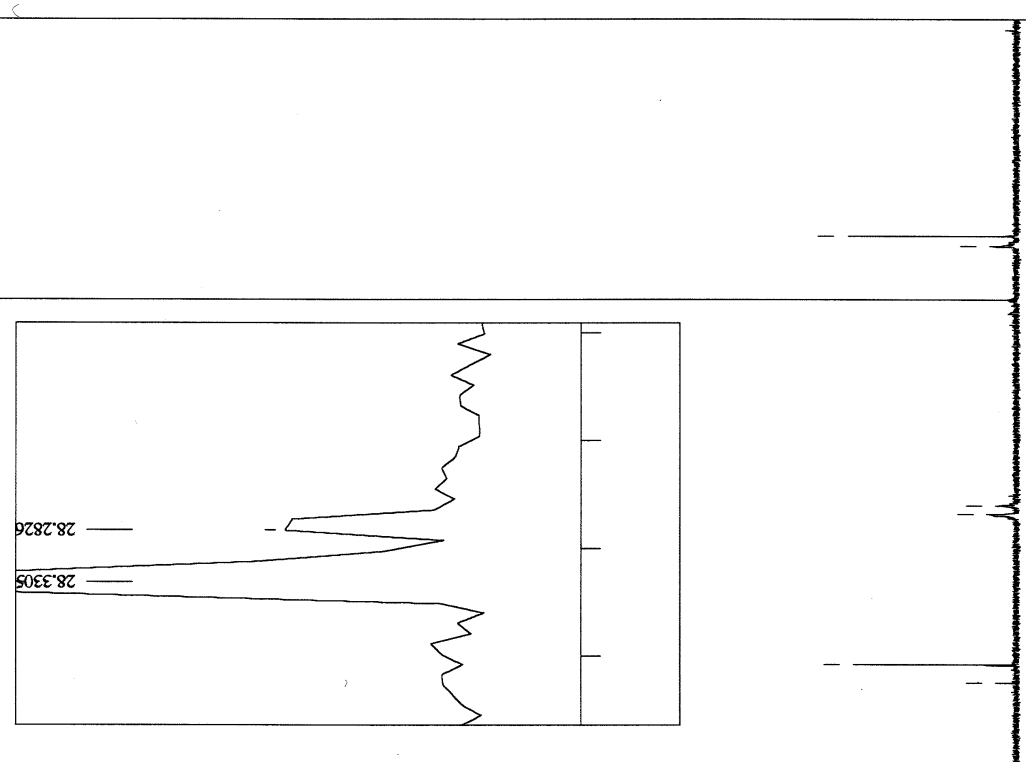

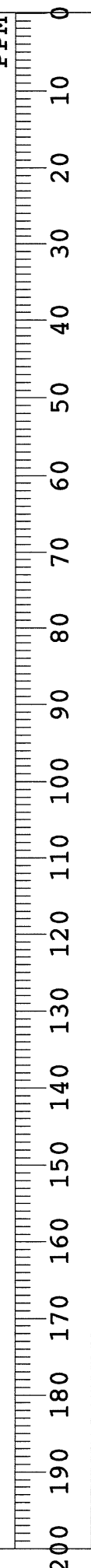




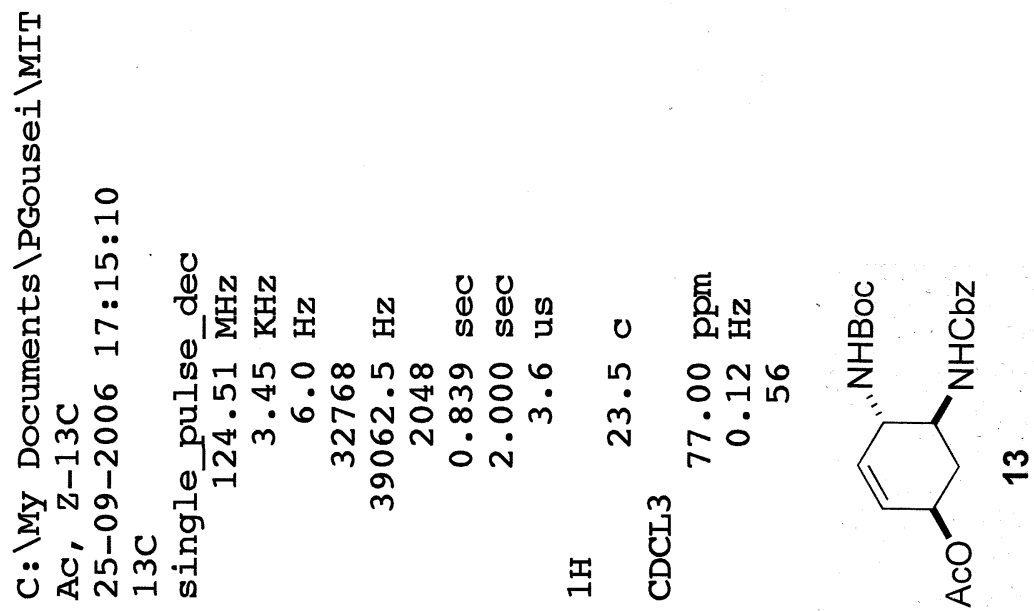

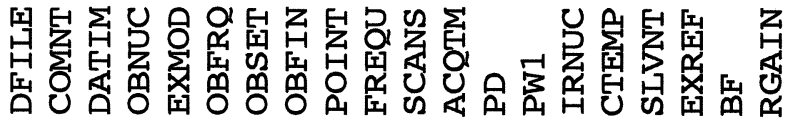

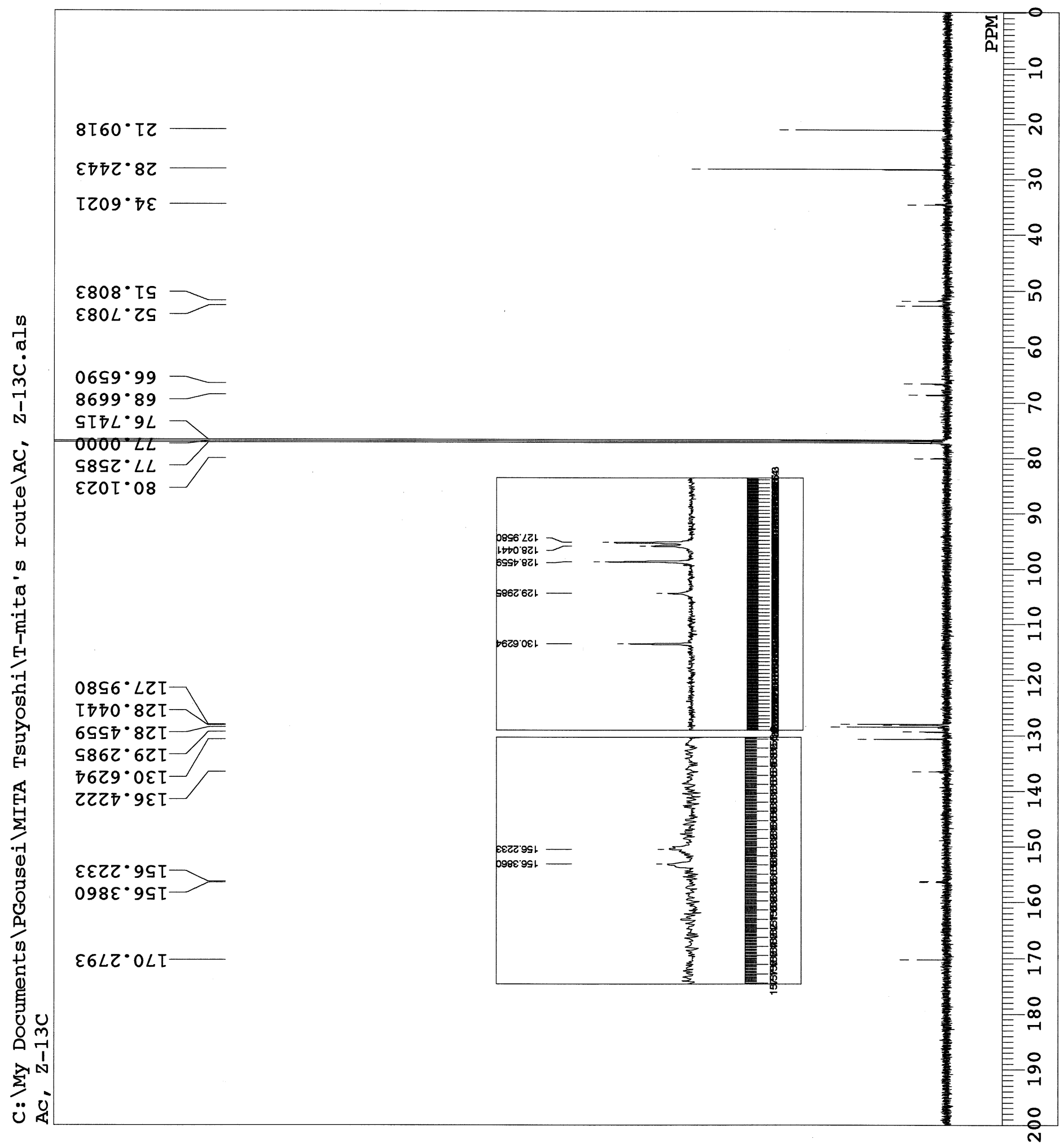


皆罗

0

ธ

ठ․․

设云它

若

국

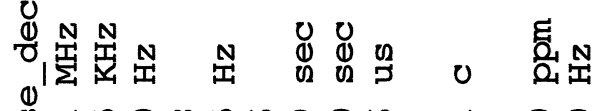

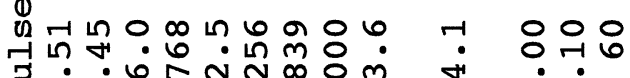

엉응

ด

事

$\leqslant 0100$

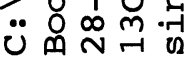

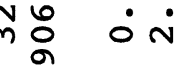

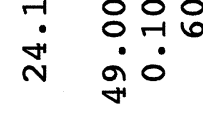

踏

$\bar{m}$

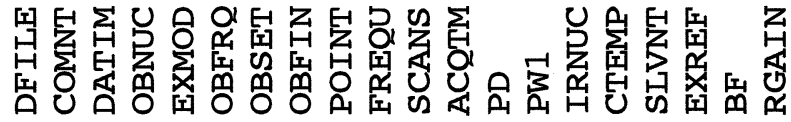

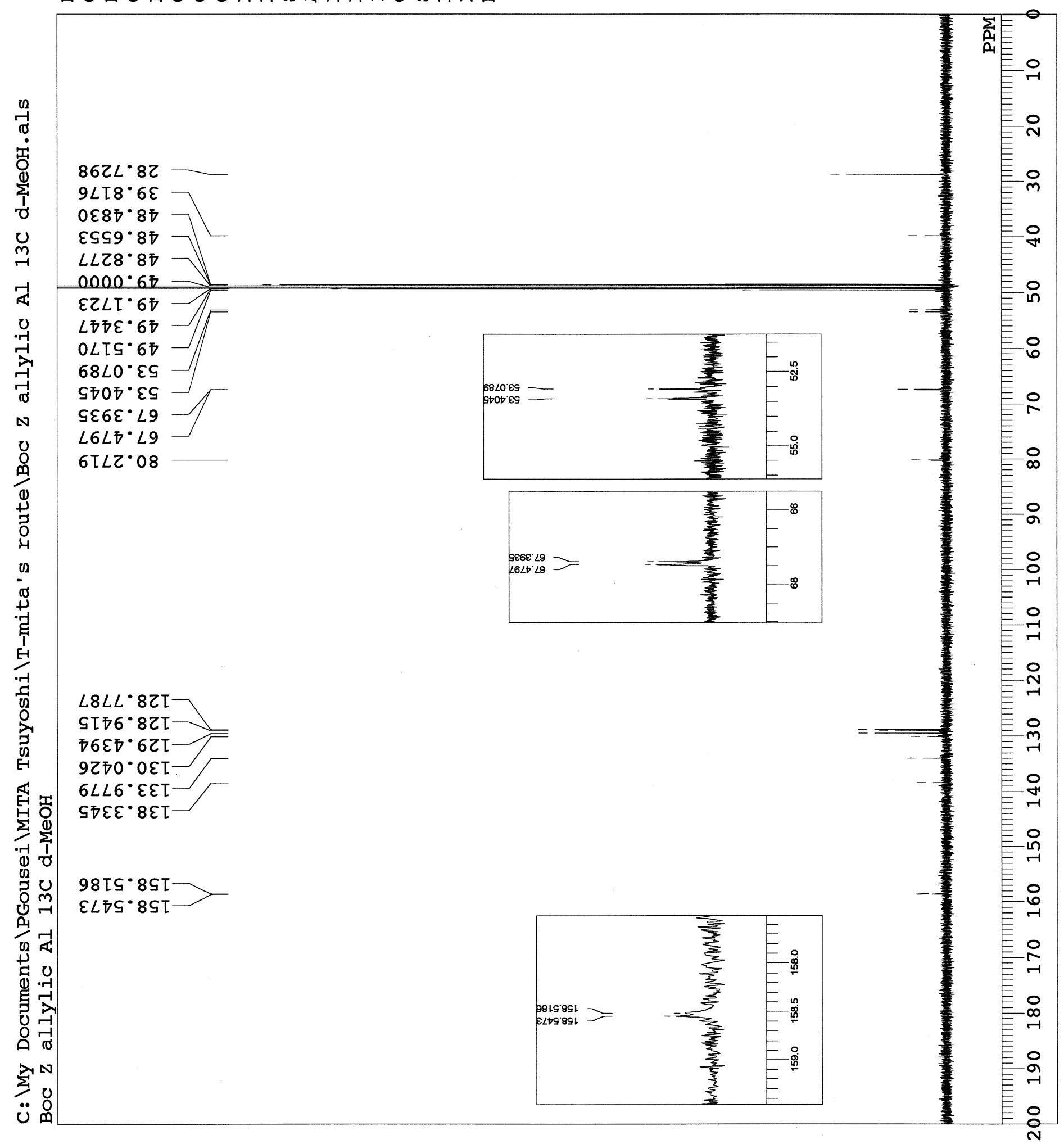


텁

○ั

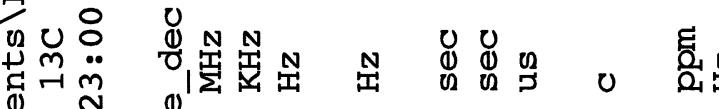

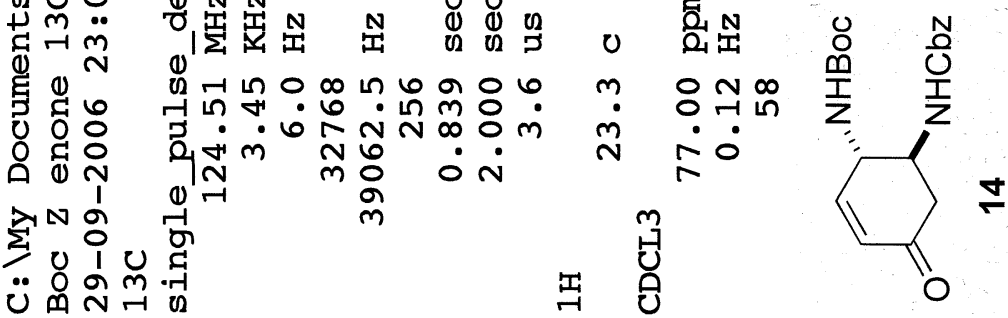

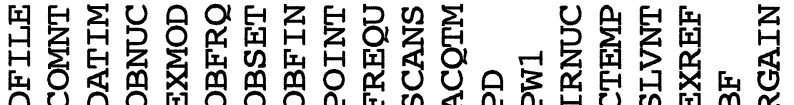

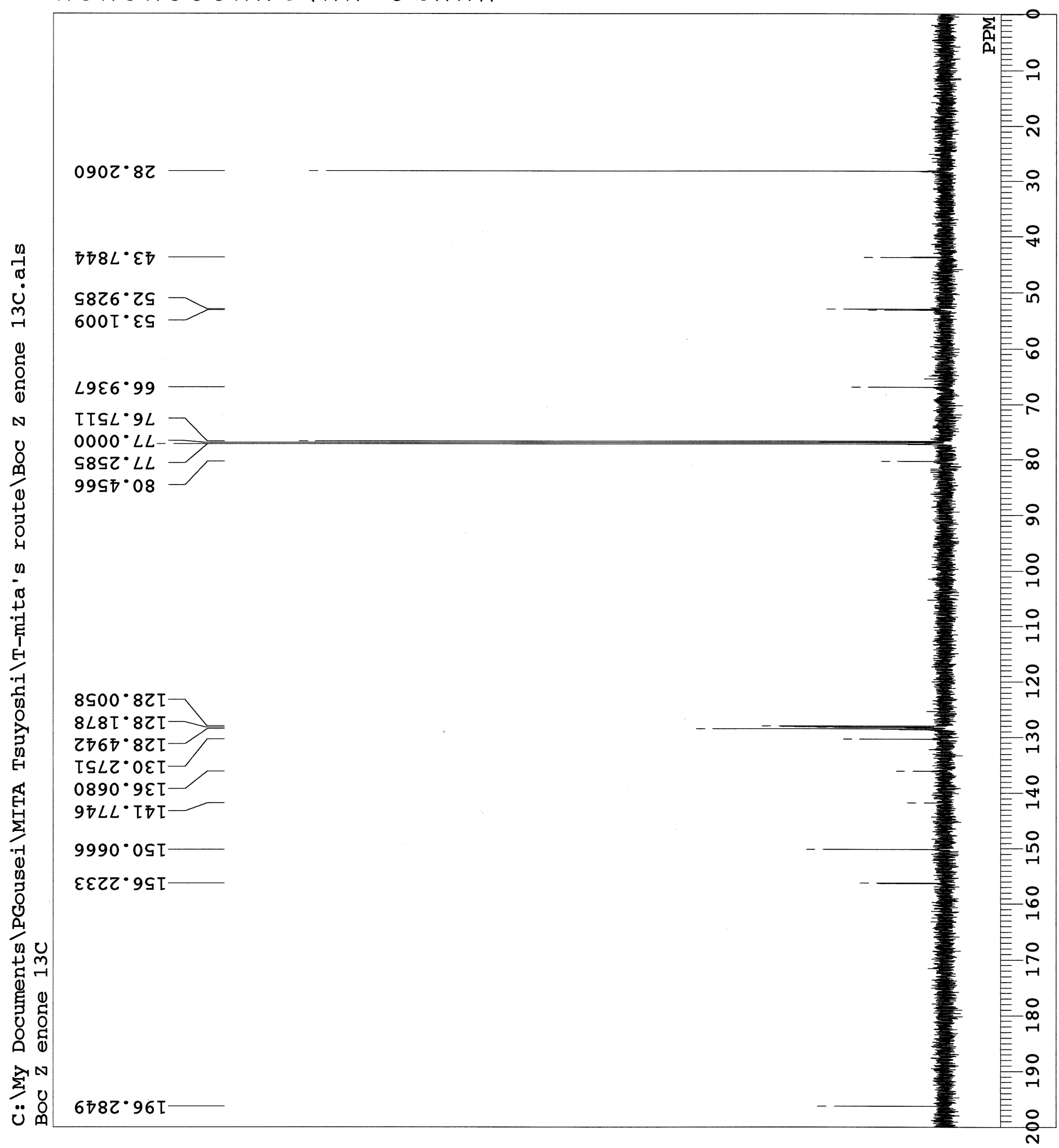


毕

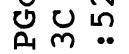

v $\ddot{\sim} \ddot{m}$

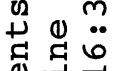

ब.

동 둥

ช ํㅇㅇ

可

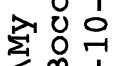

m 10

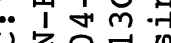

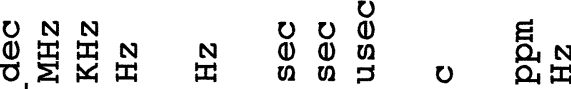
बान v in * 0 o in in $\infty$ i

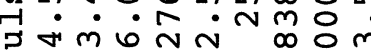

|r

aे

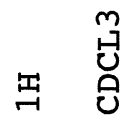

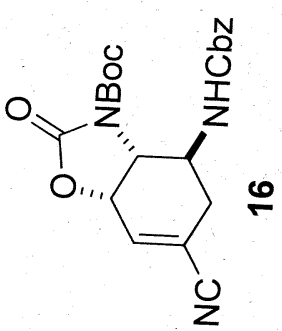

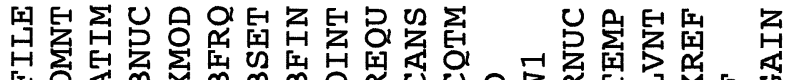

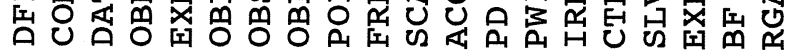

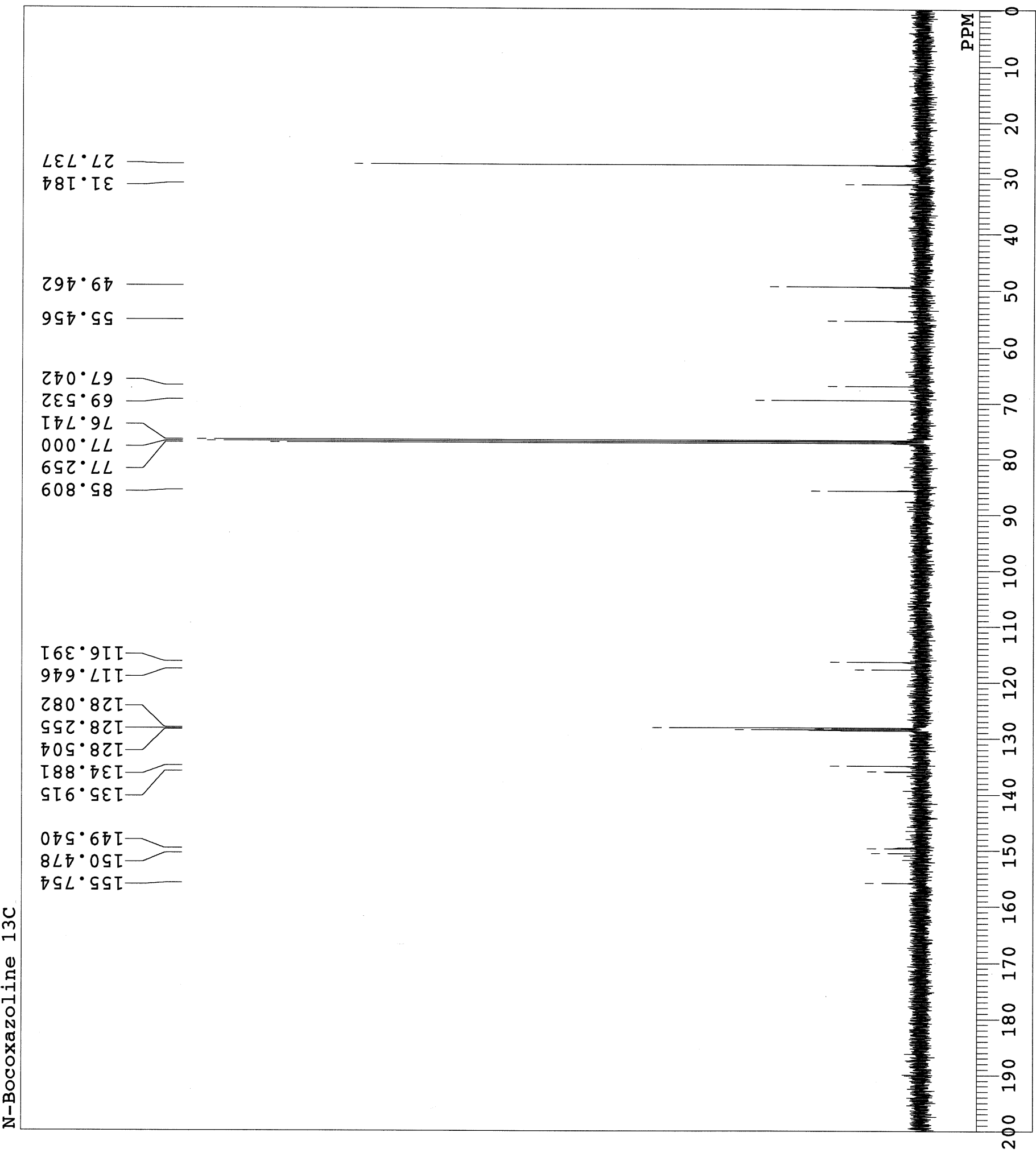




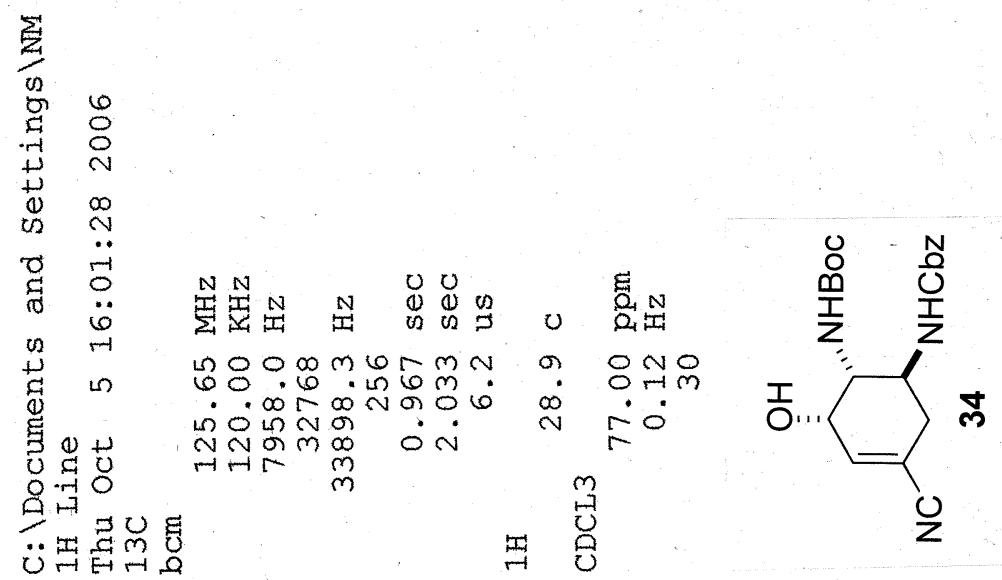

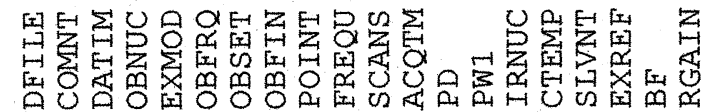


年

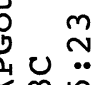

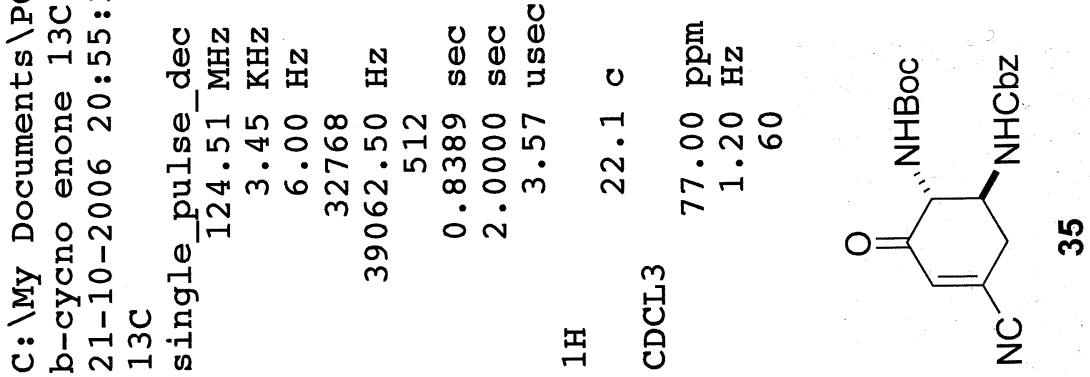

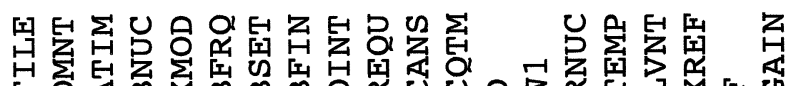

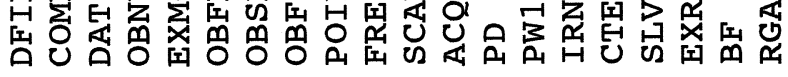

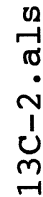

乙Е๐0 8乙

$898 \mathrm{I} \cdot 8 \mathrm{Z}$

โ89ع $\varsigma \varepsilon$

$06[8 \cdot \varepsilon G$

$68 Z I \cdot 09$

$[098 \cdot 99$

$S[\nabla L \cdot 9$

$0000^{\circ}$.tz

$\varsigma 8 \subseteq \tau^{\circ} L L$

$008 Z \cdot[8$

$288 L \cdot G \tau T$

乙298・ LZI

ह०દ $\cdot 8 Z I$

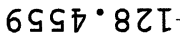

$006 \nabla^{\circ} 6 Z$ L

$\angle 960^{\circ} 9 \varepsilon I$

9 II $L \varepsilon I$

$6090 \cdot 9 \mathrm{SI}$

$\angle 6 Z \nabla^{\circ} \angle S T$

证

n

t

莺

ค

ह

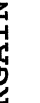


荘

O

a

ขै द्व

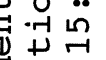

0

훙ㅇ

คัญ ำ

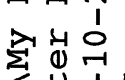

+10 .

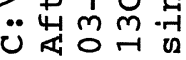

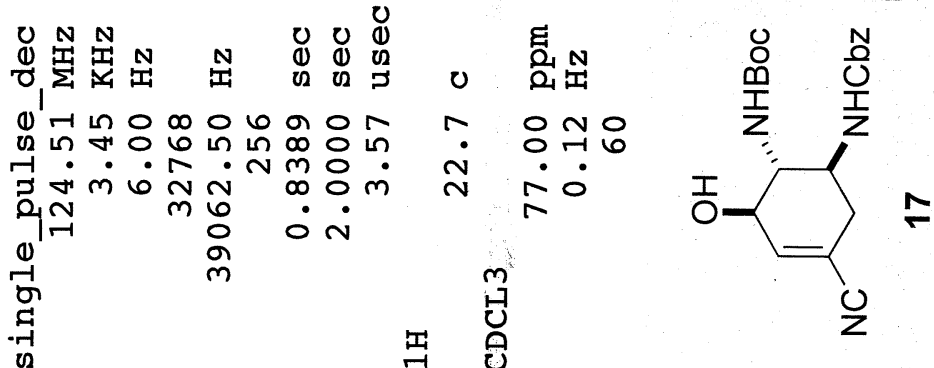

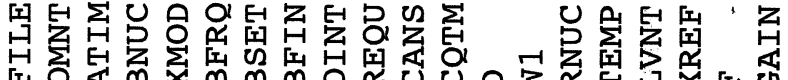

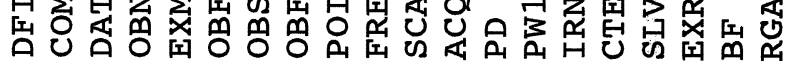

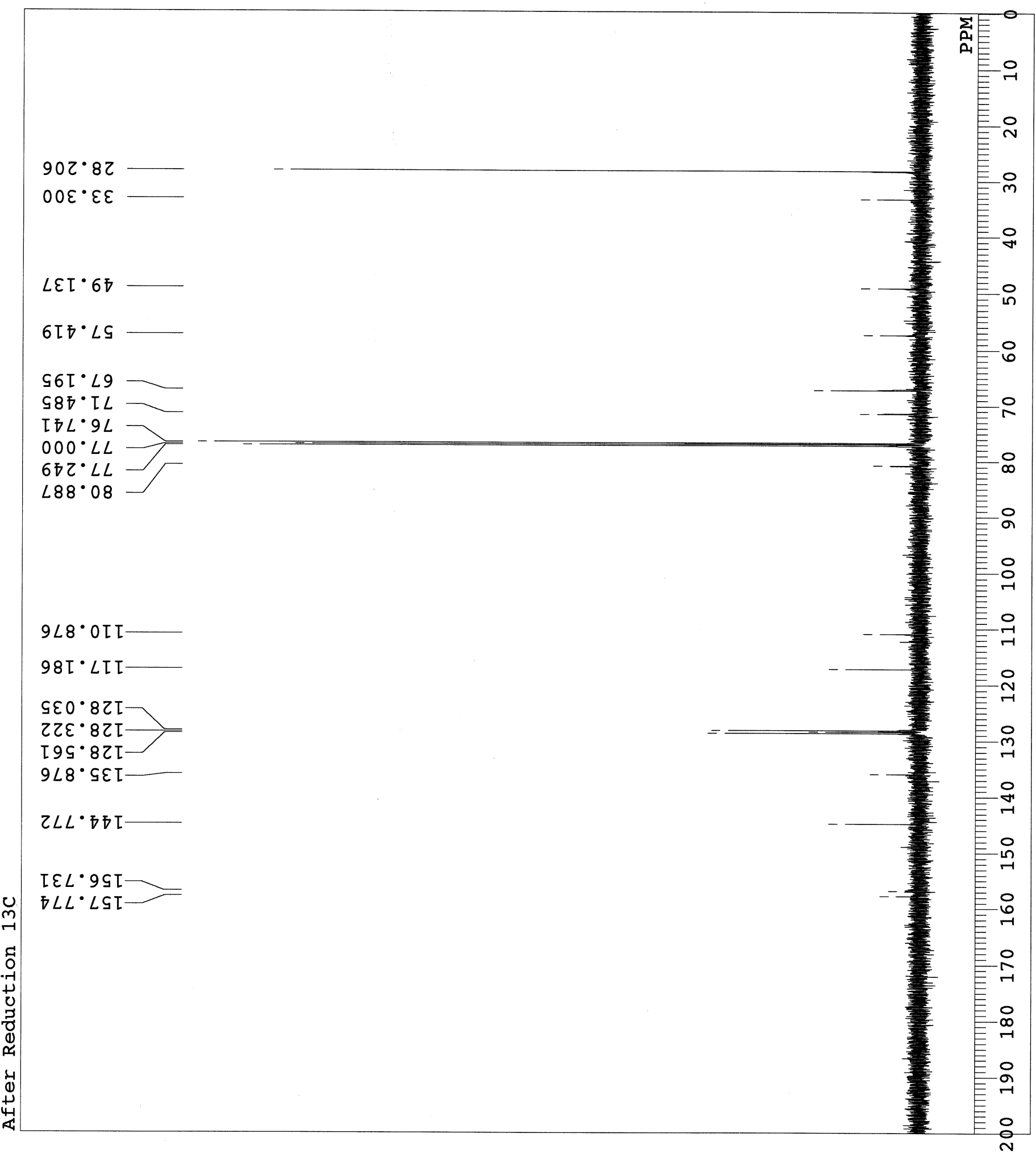




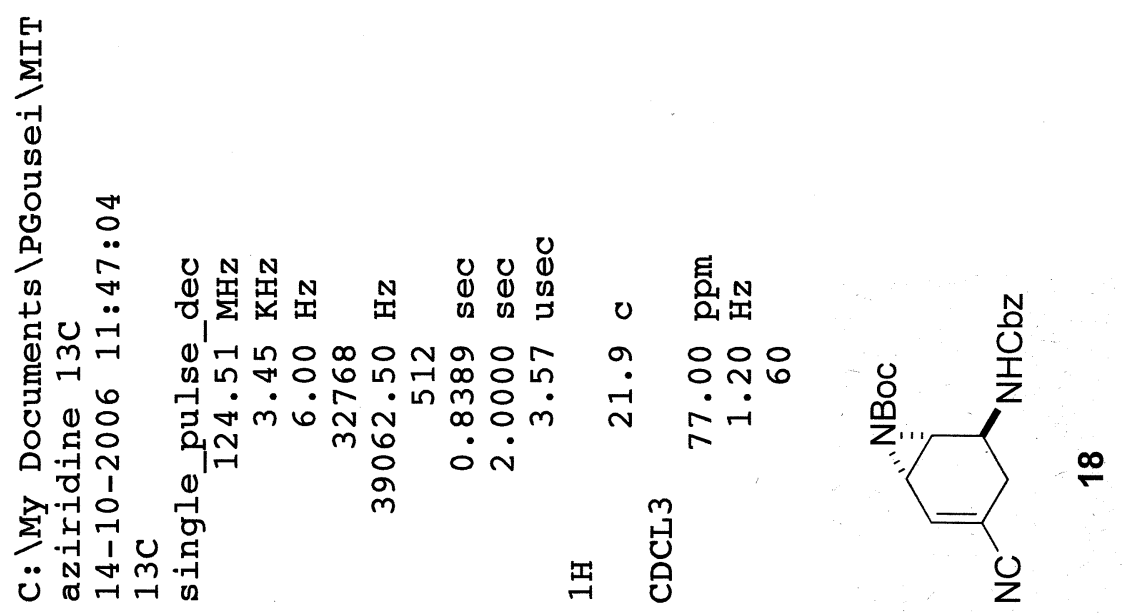

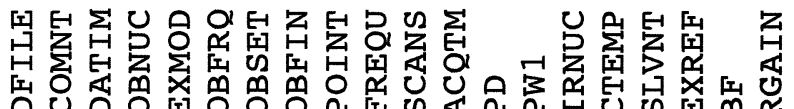

ISLL・LZ

$\varepsilon \angle L 6^{\circ} 6 Z$

$\tau \varepsilon \angle 8^{\circ}[\varepsilon$

$8 \subseteq L Z \cdot I$

$\varepsilon \varepsilon 8 L \cdot \tau \hbar$

$\varepsilon 乙 9 乙 \cdot \angle 9$

I I $L \cdot 9 L$

$0000^{\circ} L L$

$98 \mathrm{G} \tau^{\circ} L L$

9โZ৪・ Z8

O૬૬乙・ZI -

$\varepsilon \subseteq 0 I \cdot 8 \tau I-$

$8 \succsim \subseteq て \cdot 8 Z I$

$080 \sigma^{\circ} 8 Z$ L

$98 โ 9 \cdot 8 Z$ I-

06 โร. งEL-

ZZऽて・O万I-

I L LE・ S I-

$8826^{\circ} 6 \mathrm{GI}$

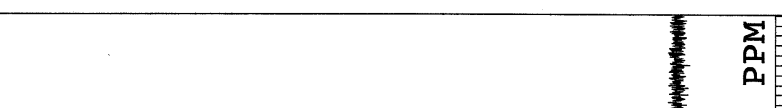


㟲

ט.

ริ

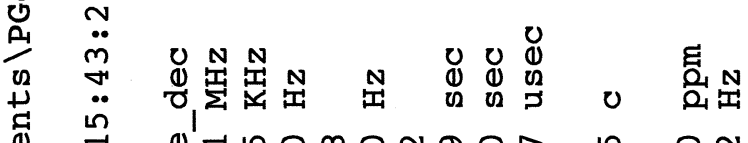

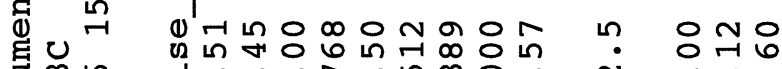
至 ขึ

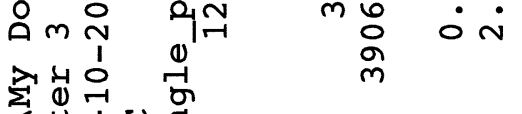

$\checkmark+1$ U

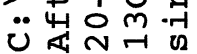

㖗苟

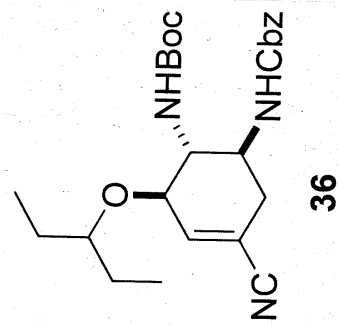

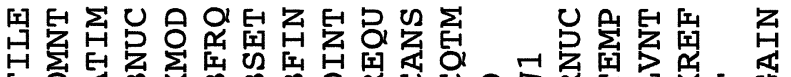

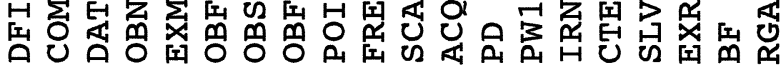

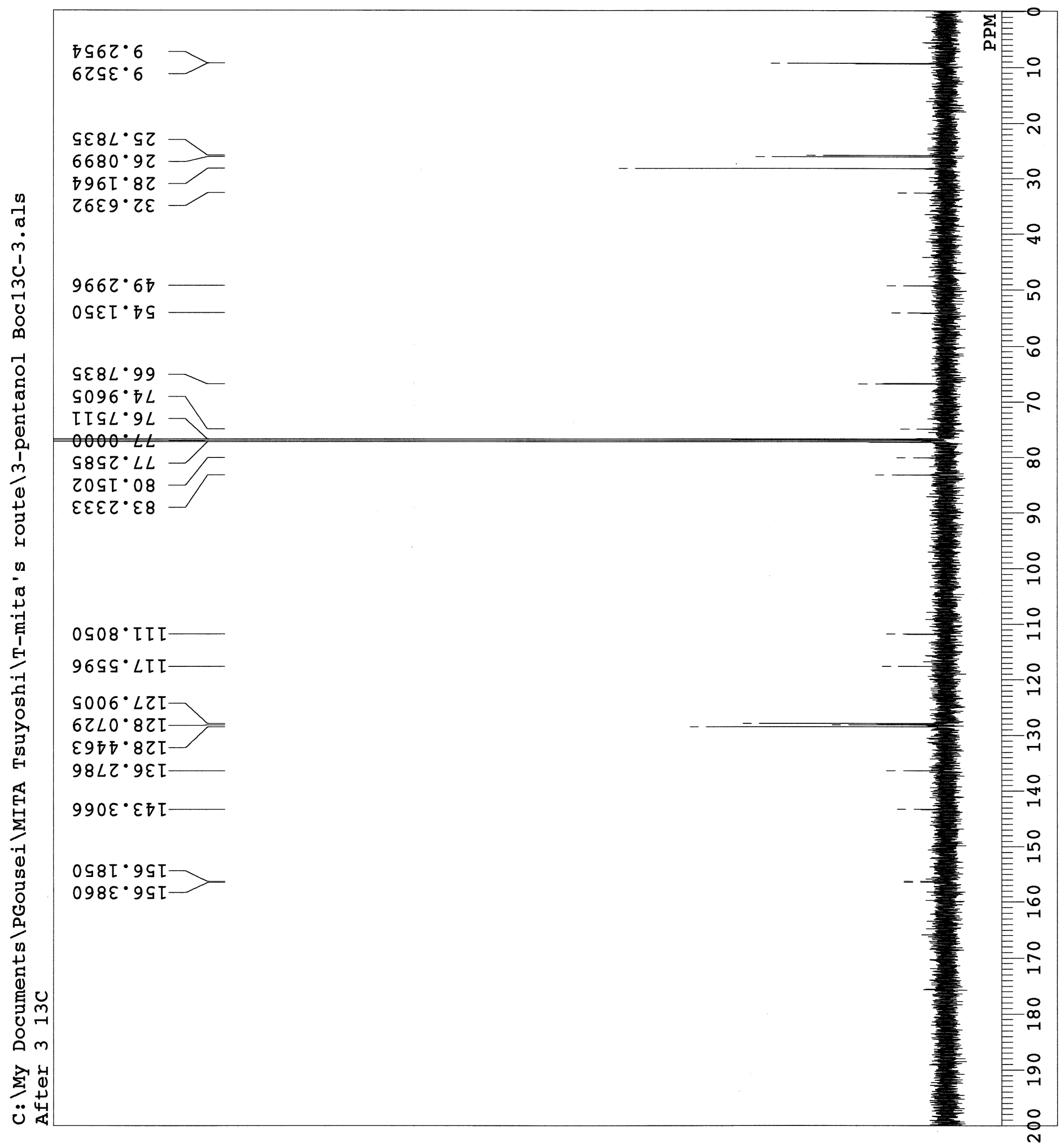


皇

苞

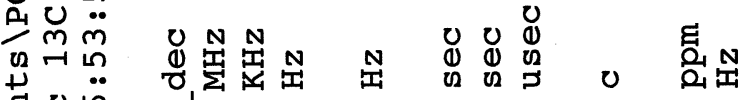

若

n

चु

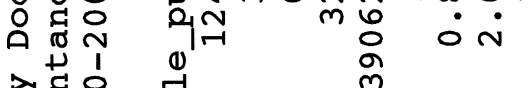

嵒 苞

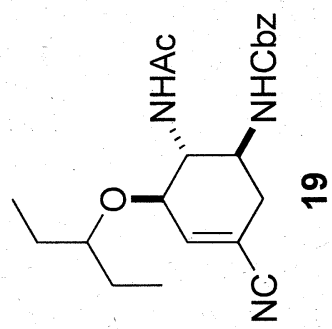

$\sum 0$

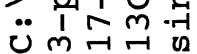

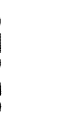

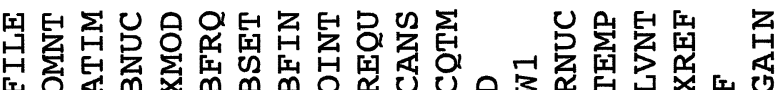

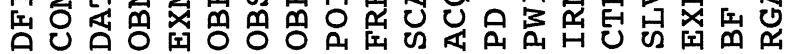

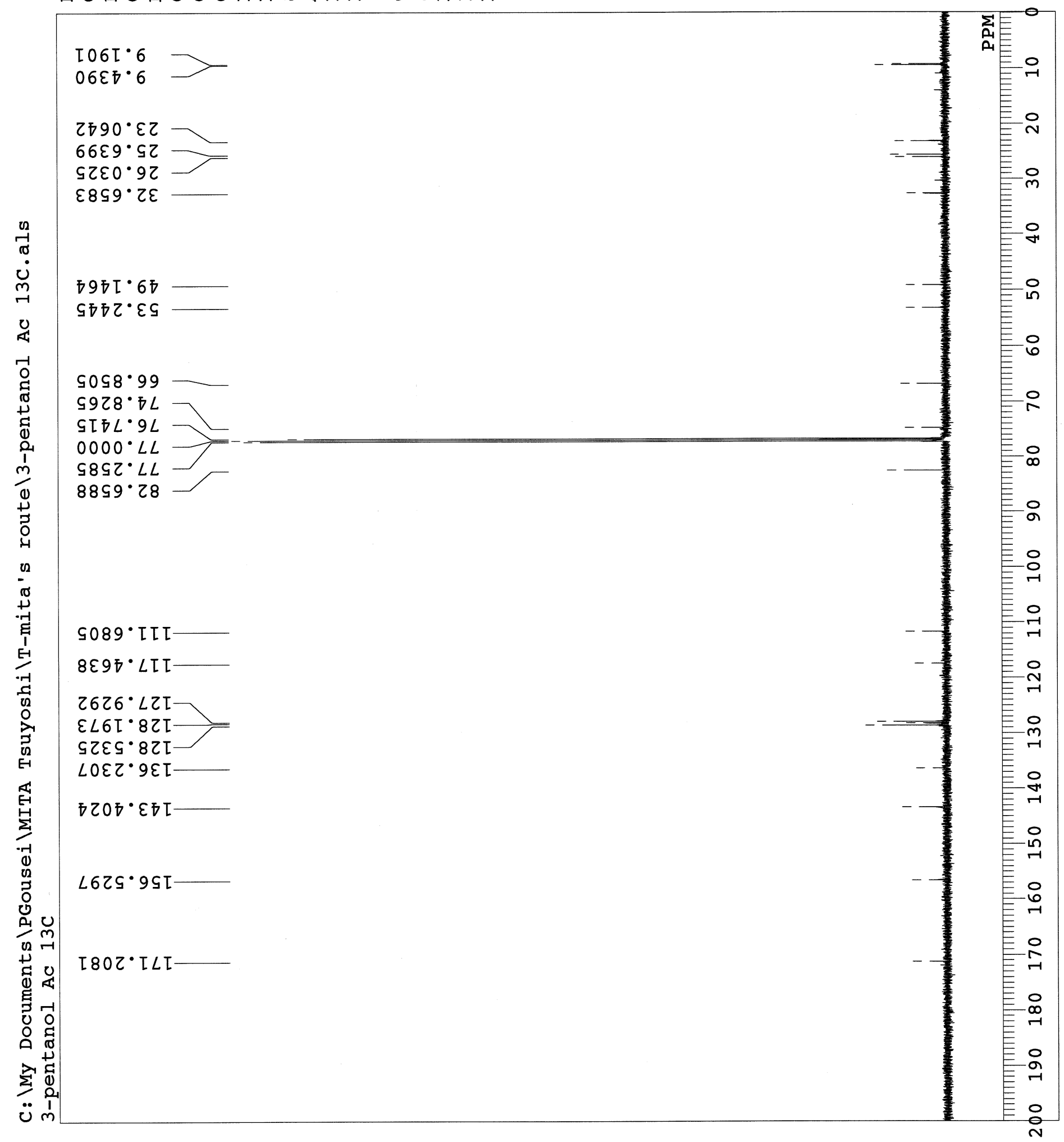




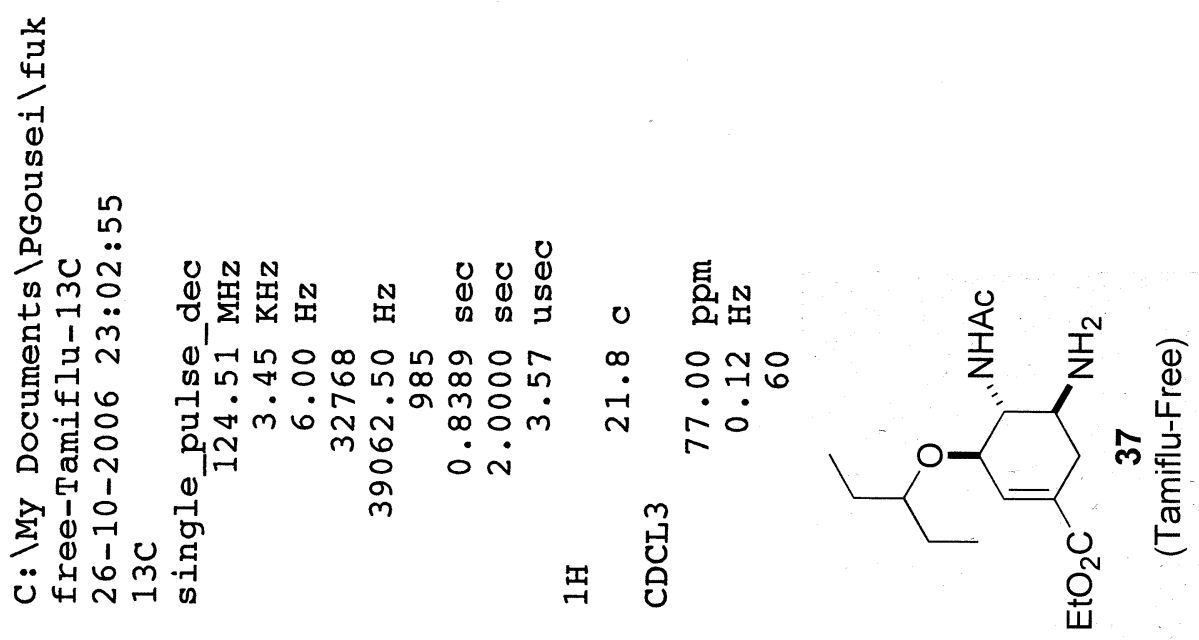

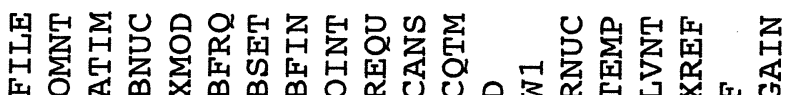

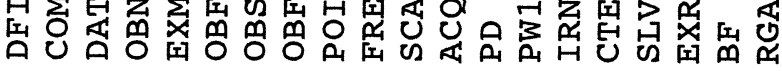

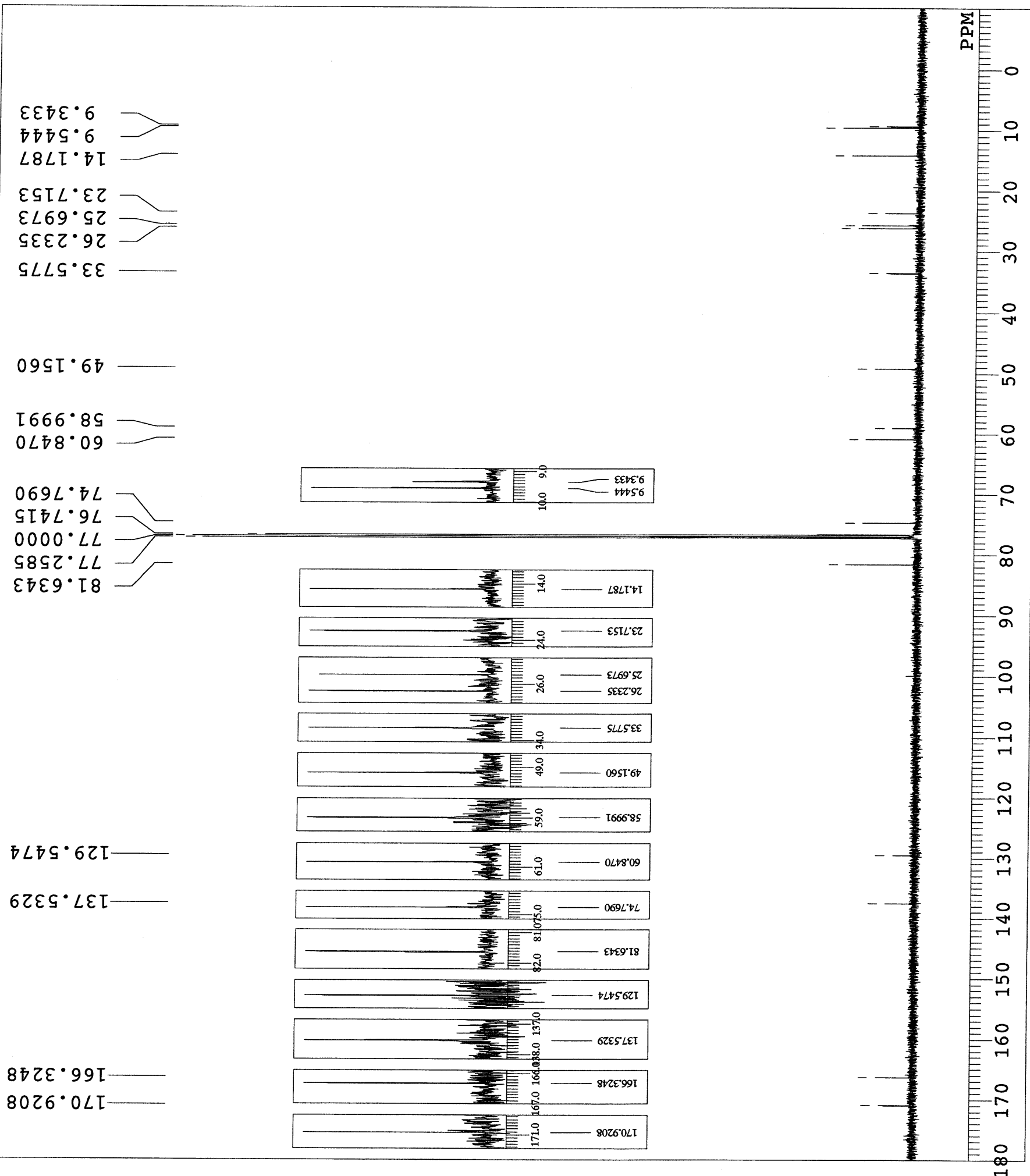




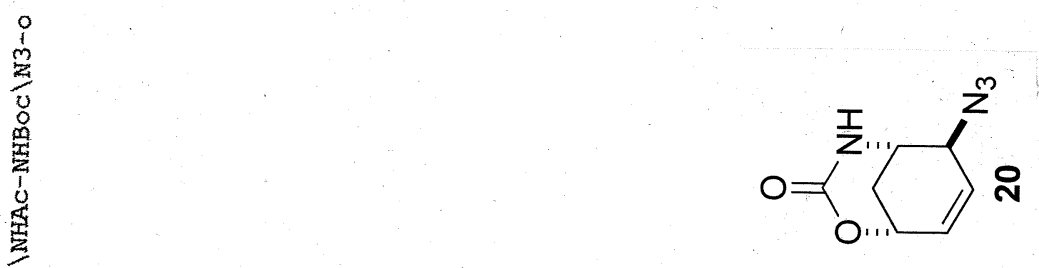

苟

\section{어료.?}

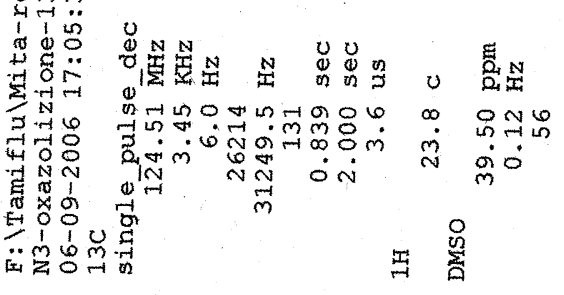

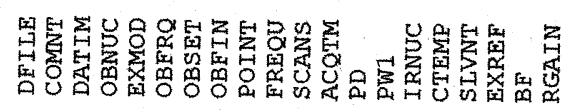

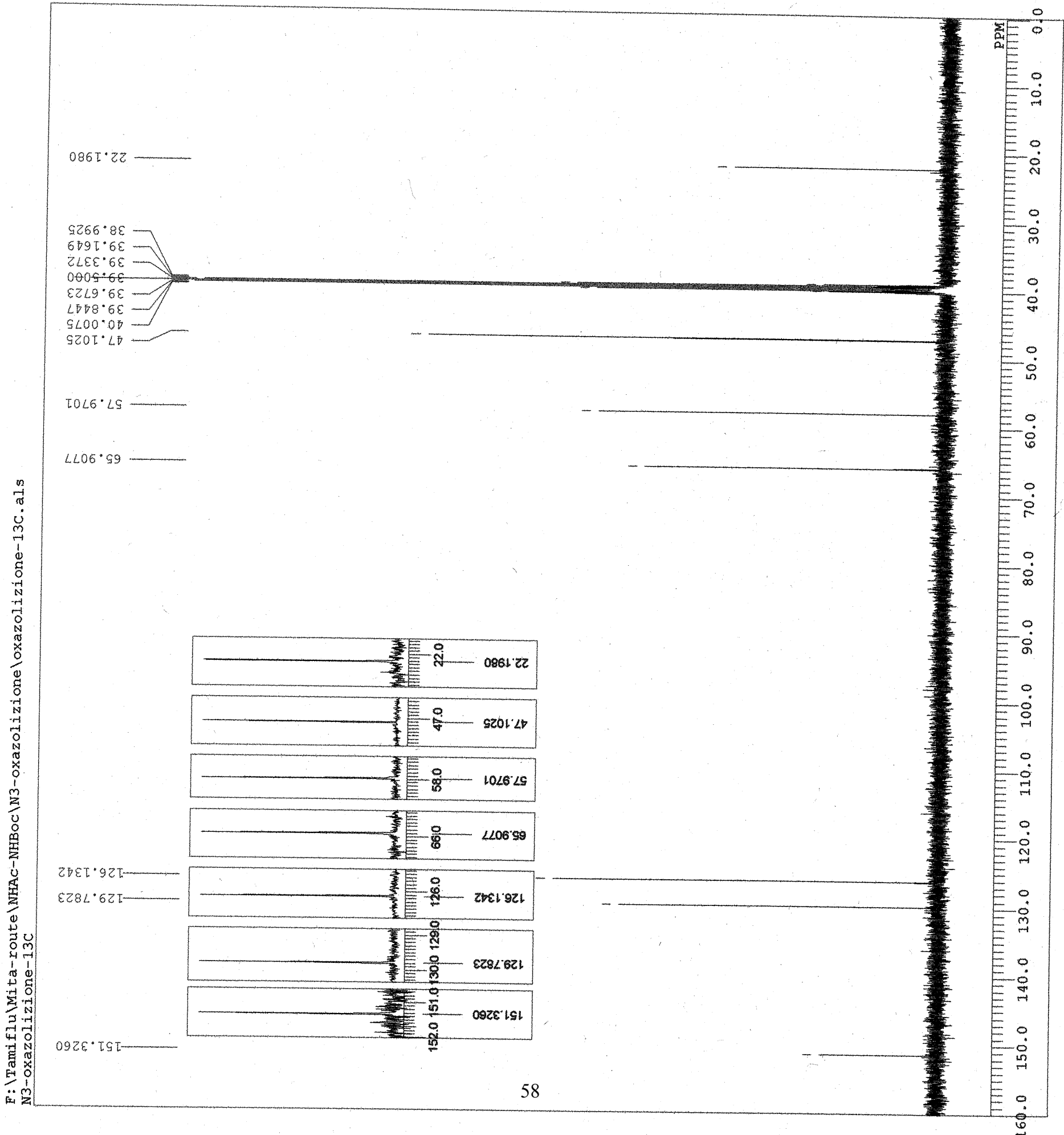




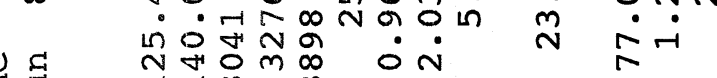

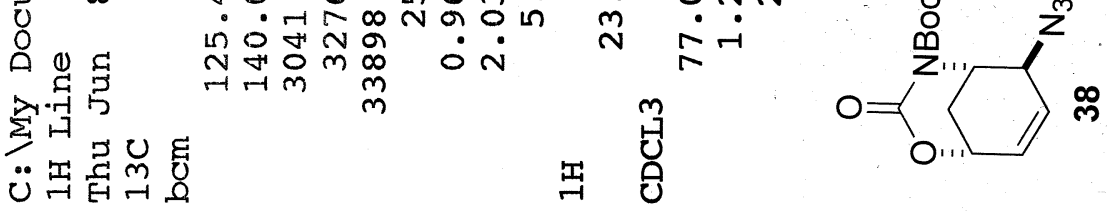

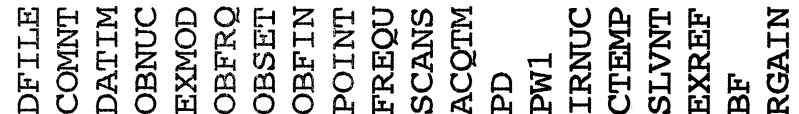

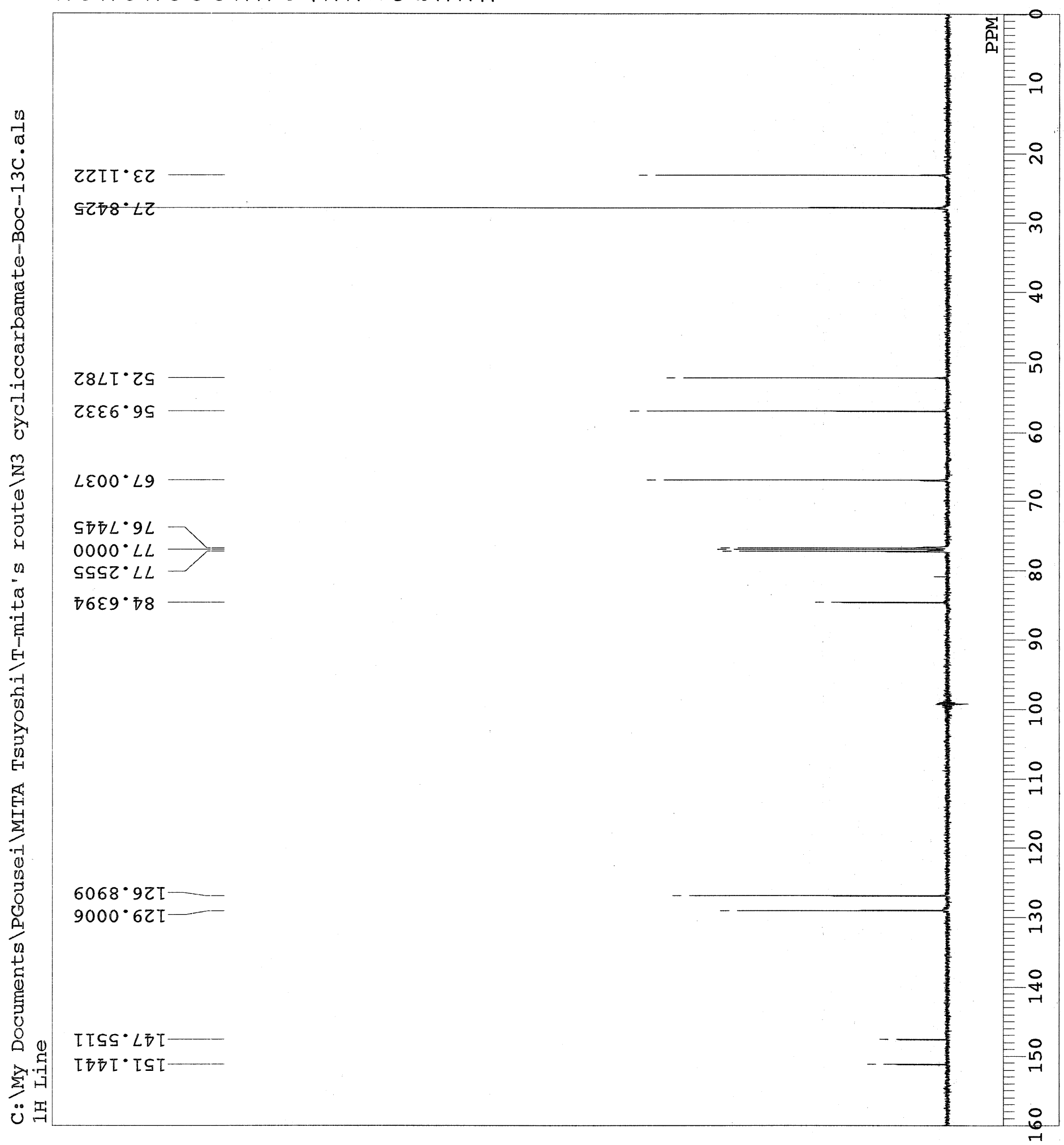


$\sum_{\substack{-1 \\ 0}}^{\text {帒 }}$

记

ช $\mathrm{N}$

110

in

(1)

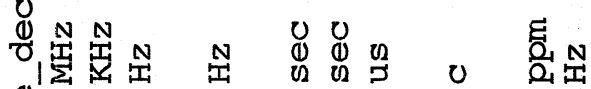

दू.

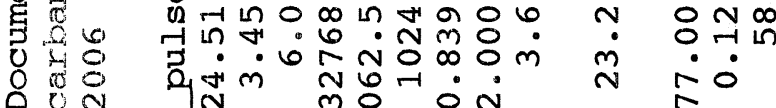

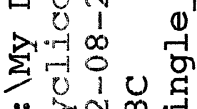

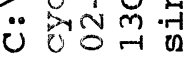

mें $\dot{0}$ N

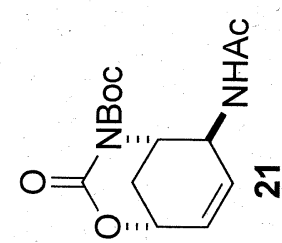

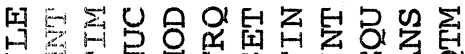

i⿱宀 犬 $\overline{\text { }}$

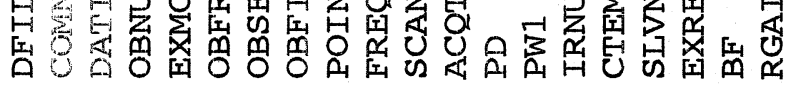

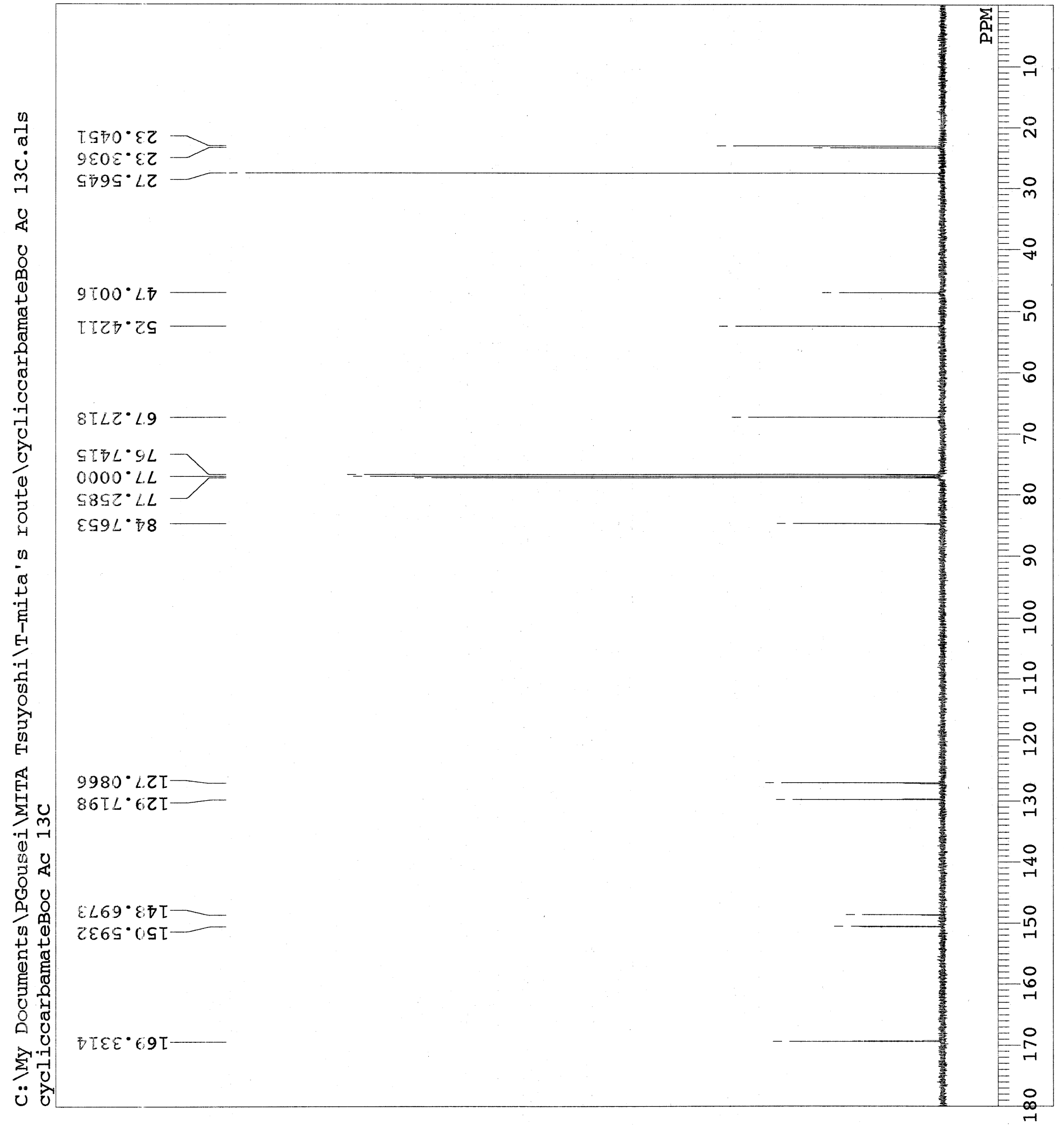




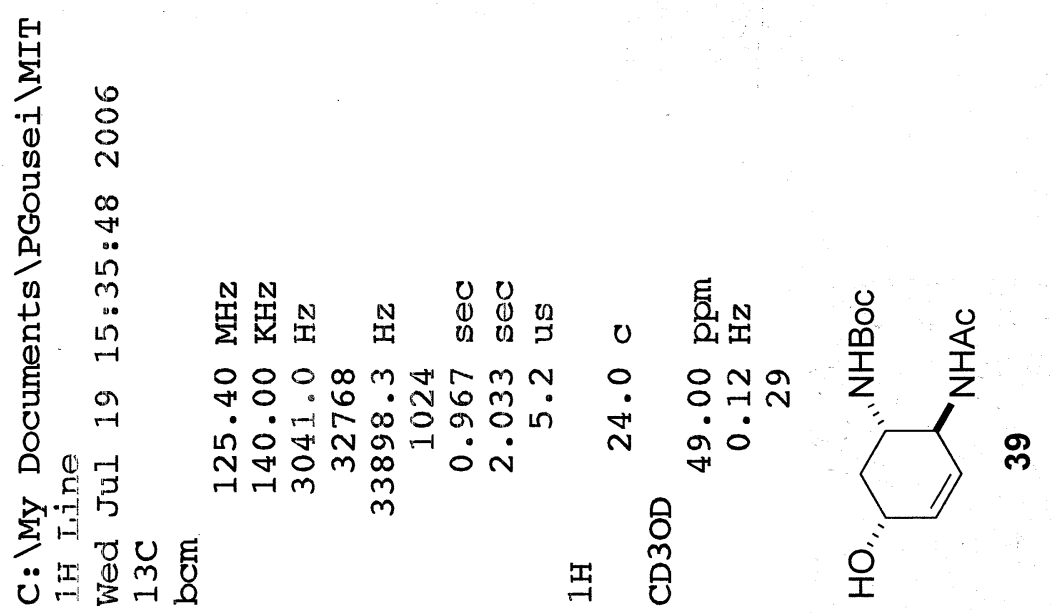

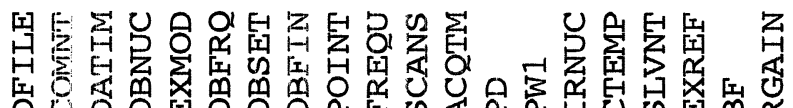

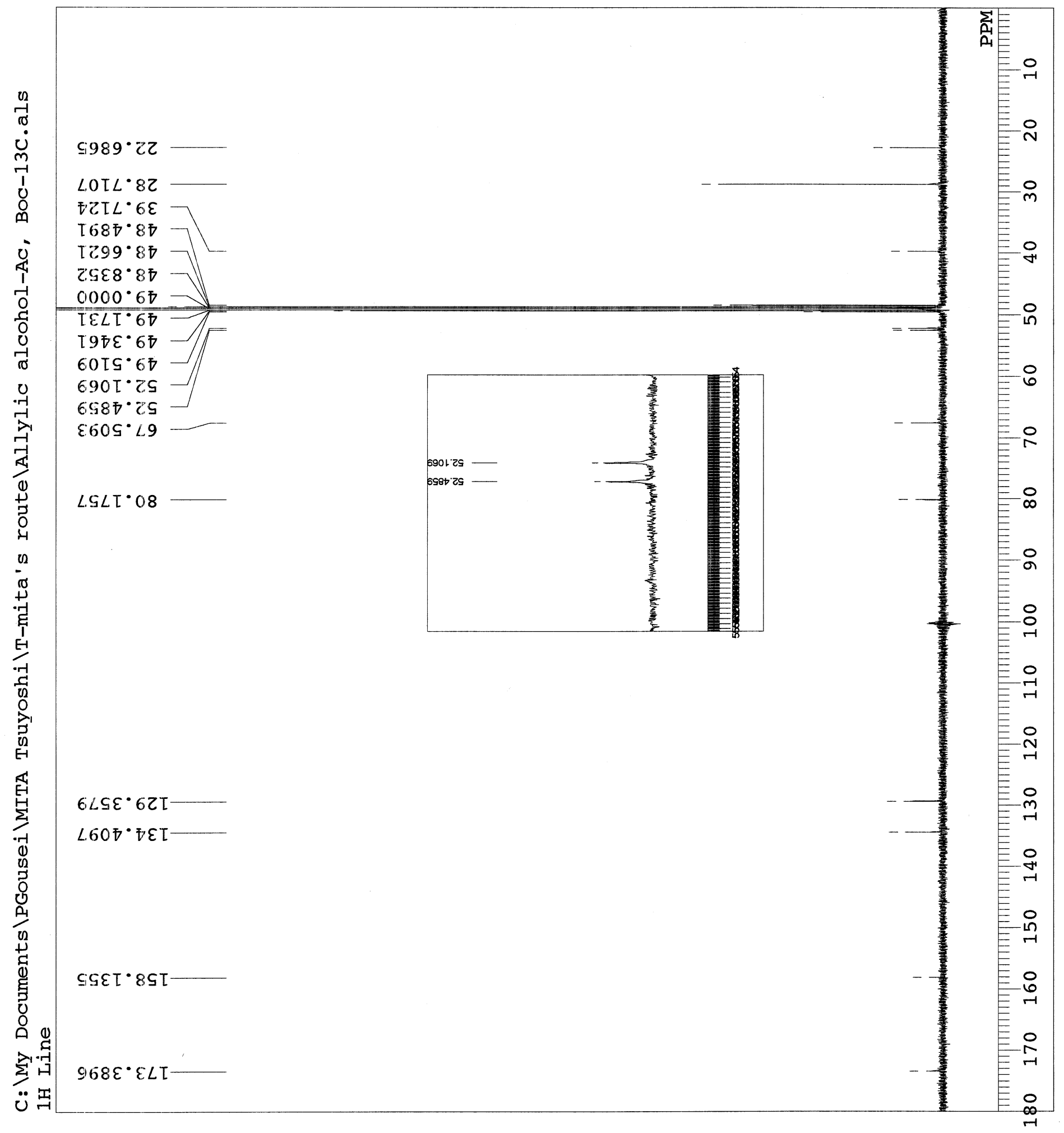




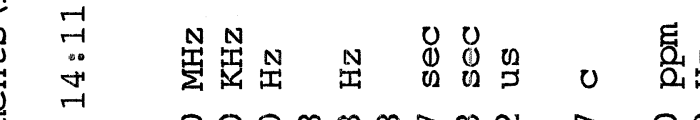

N 至

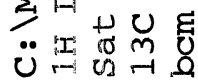

踏苞

\section{ก}

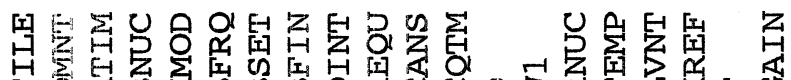

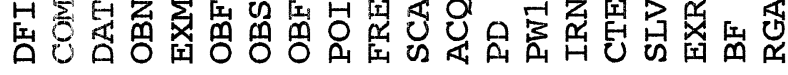

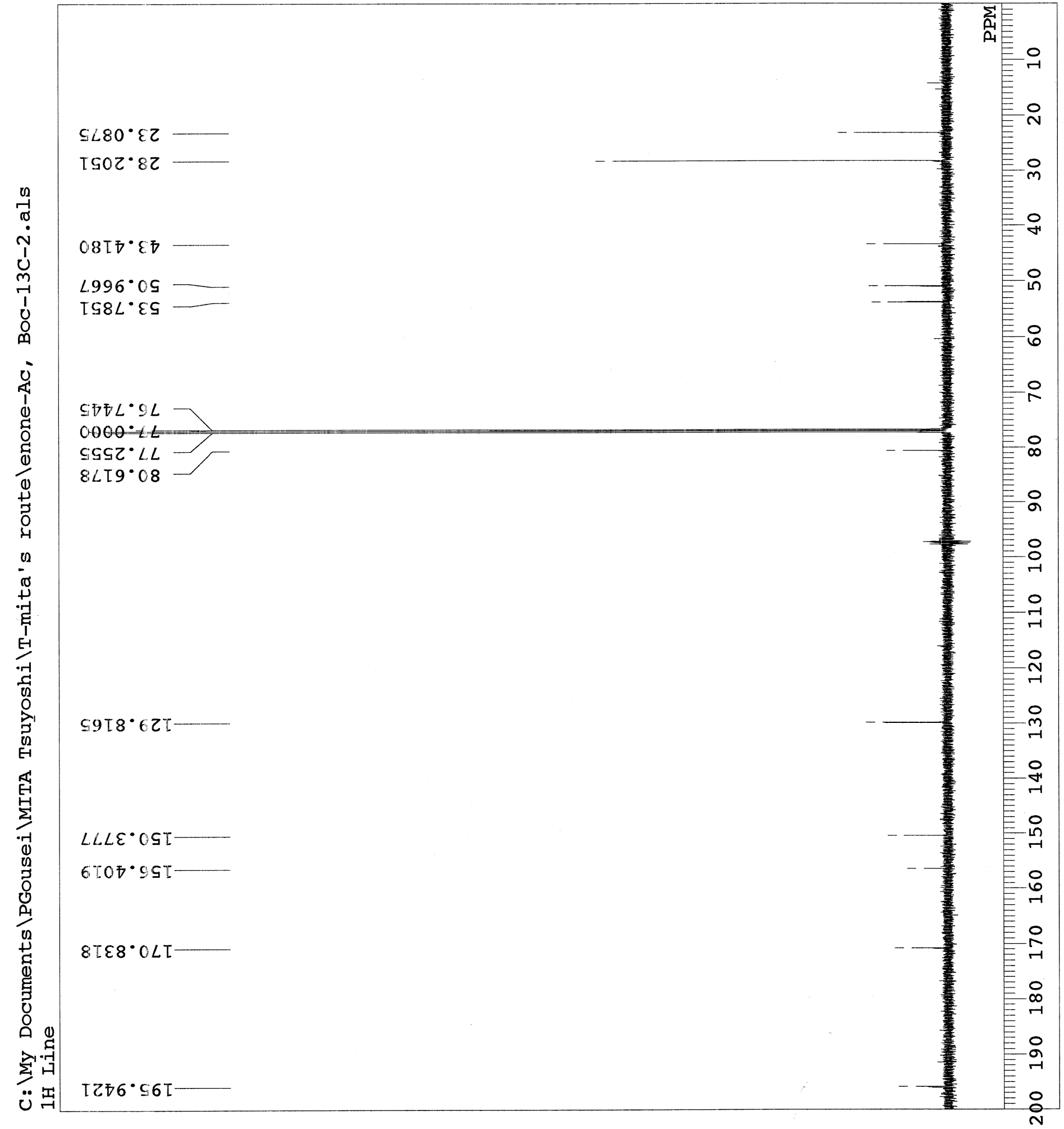




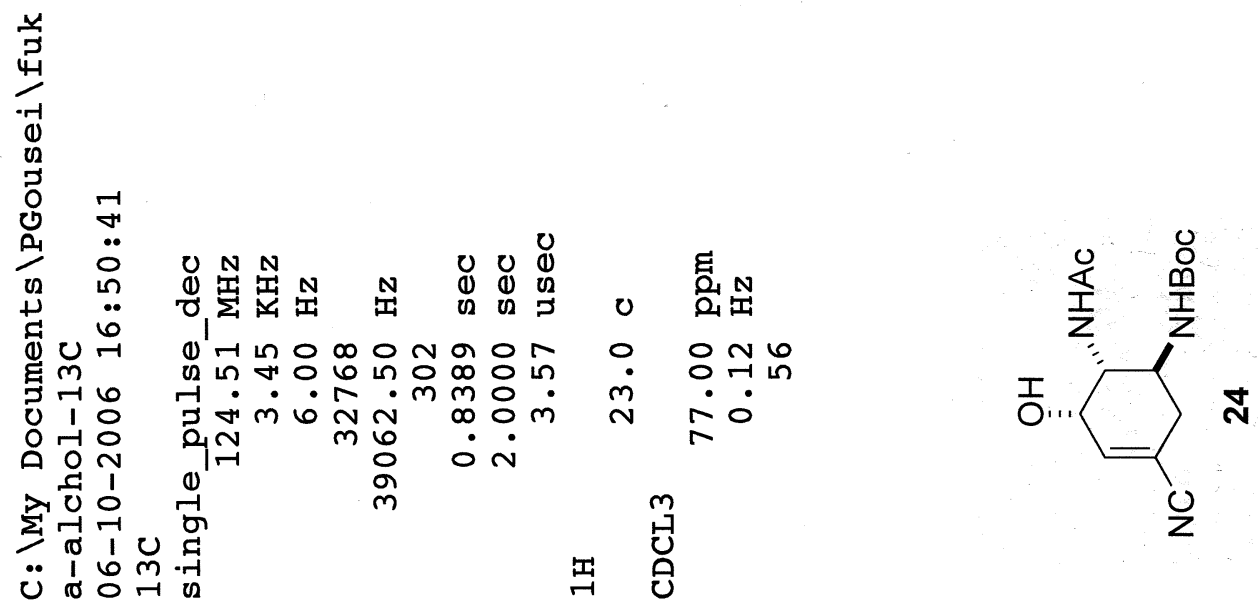

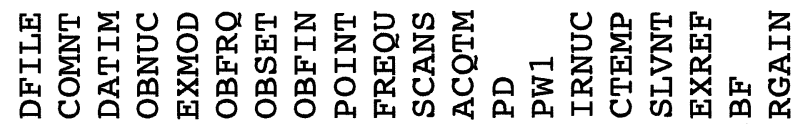

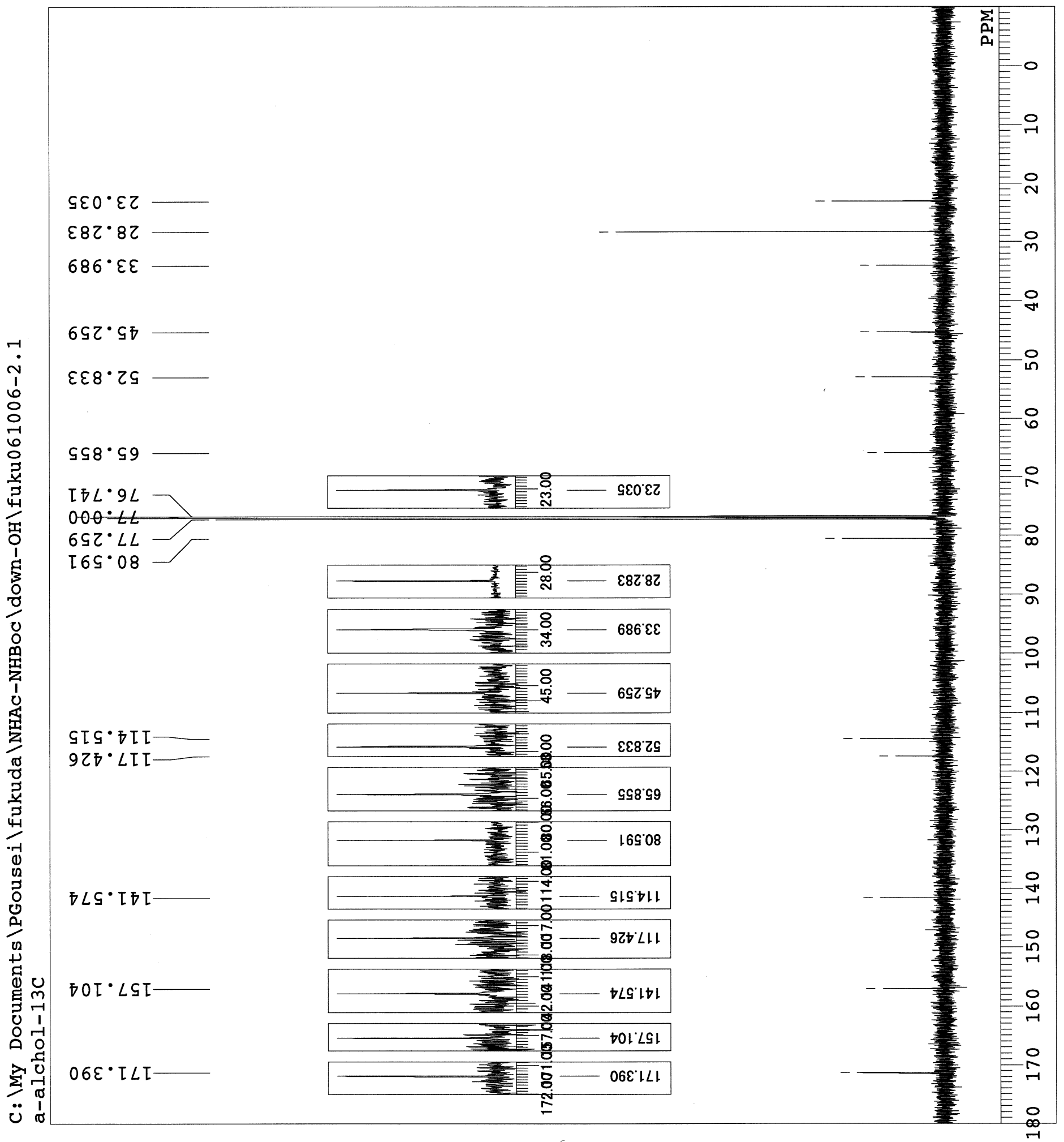



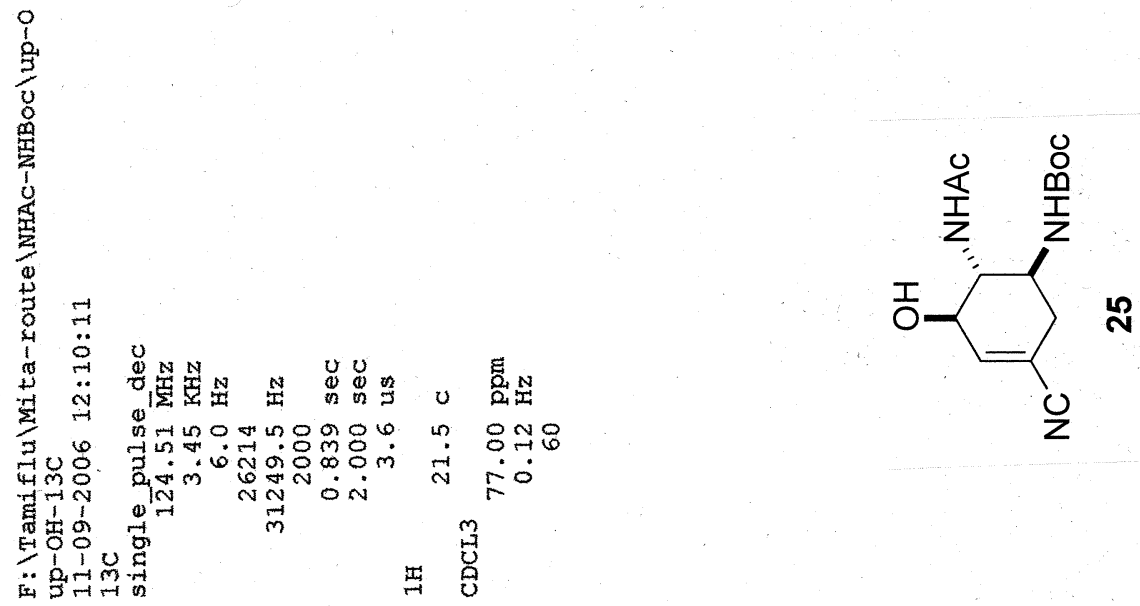

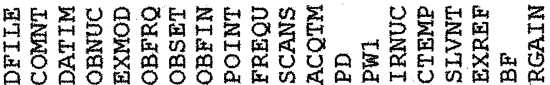

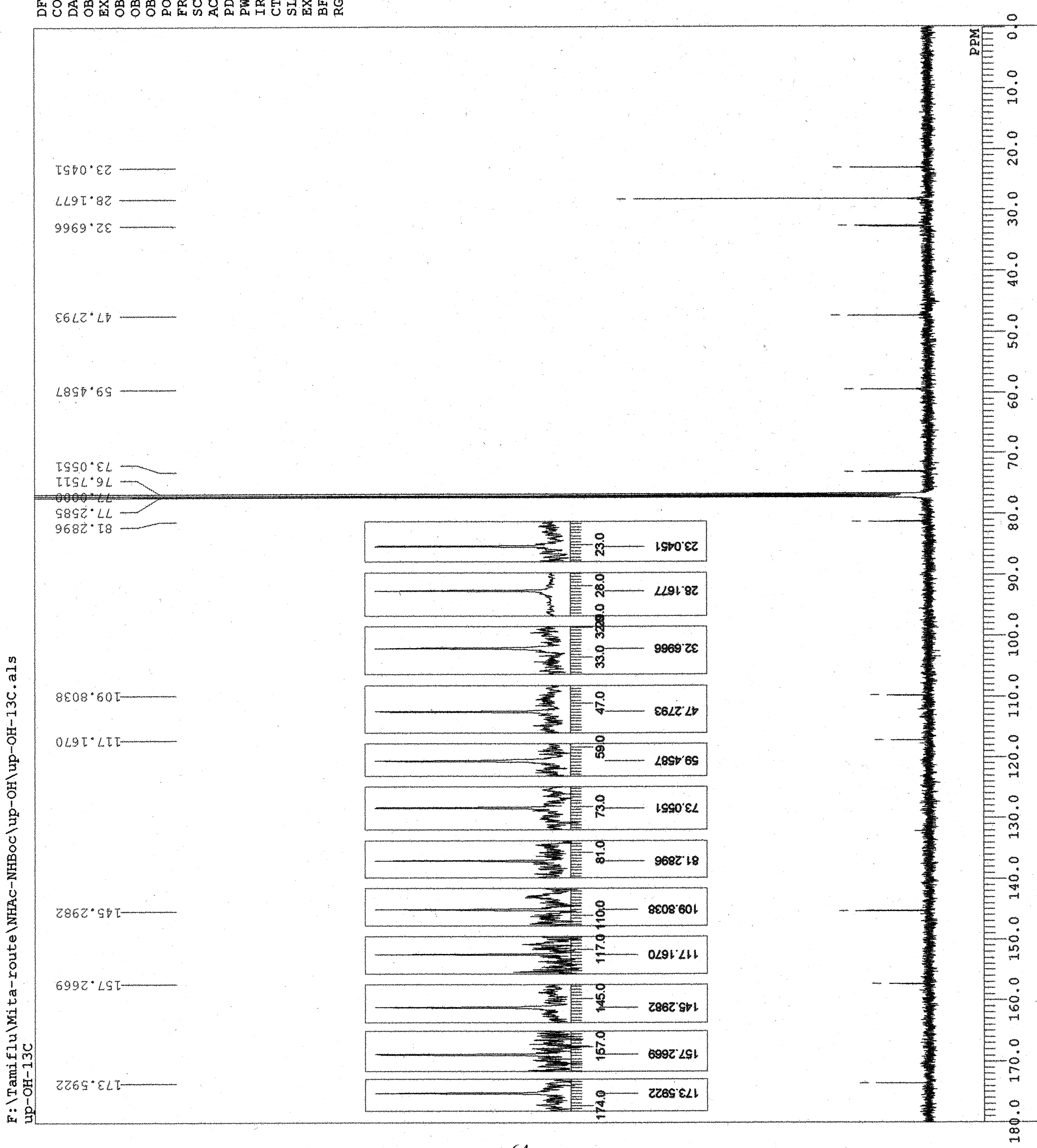




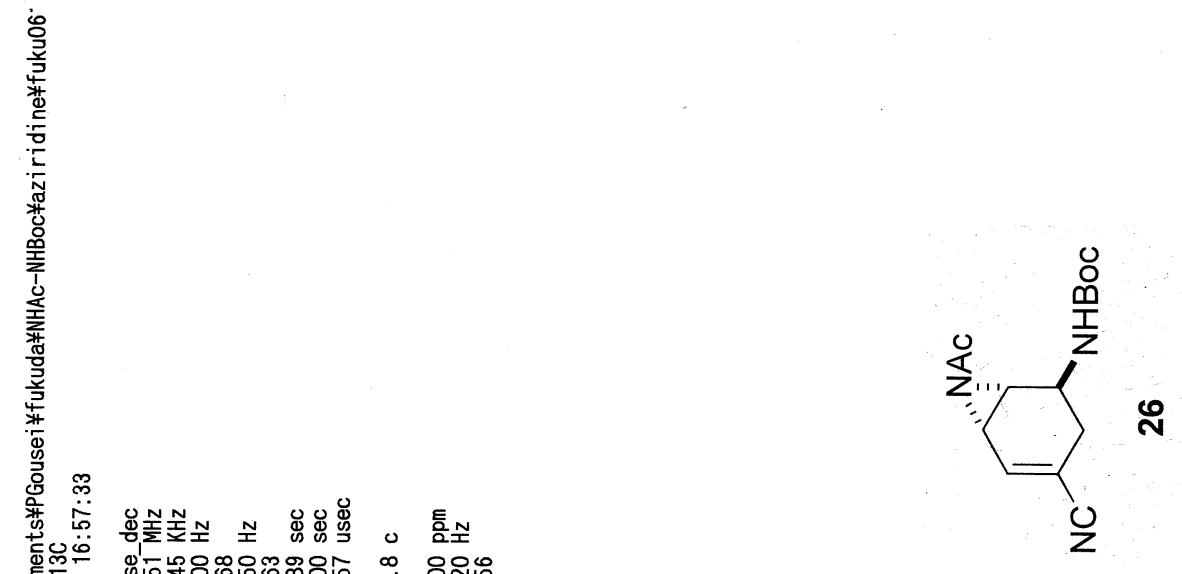

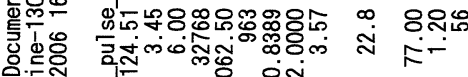

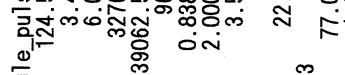

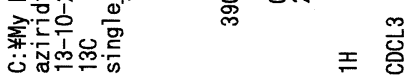

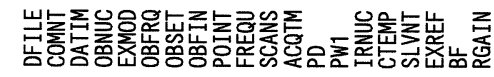

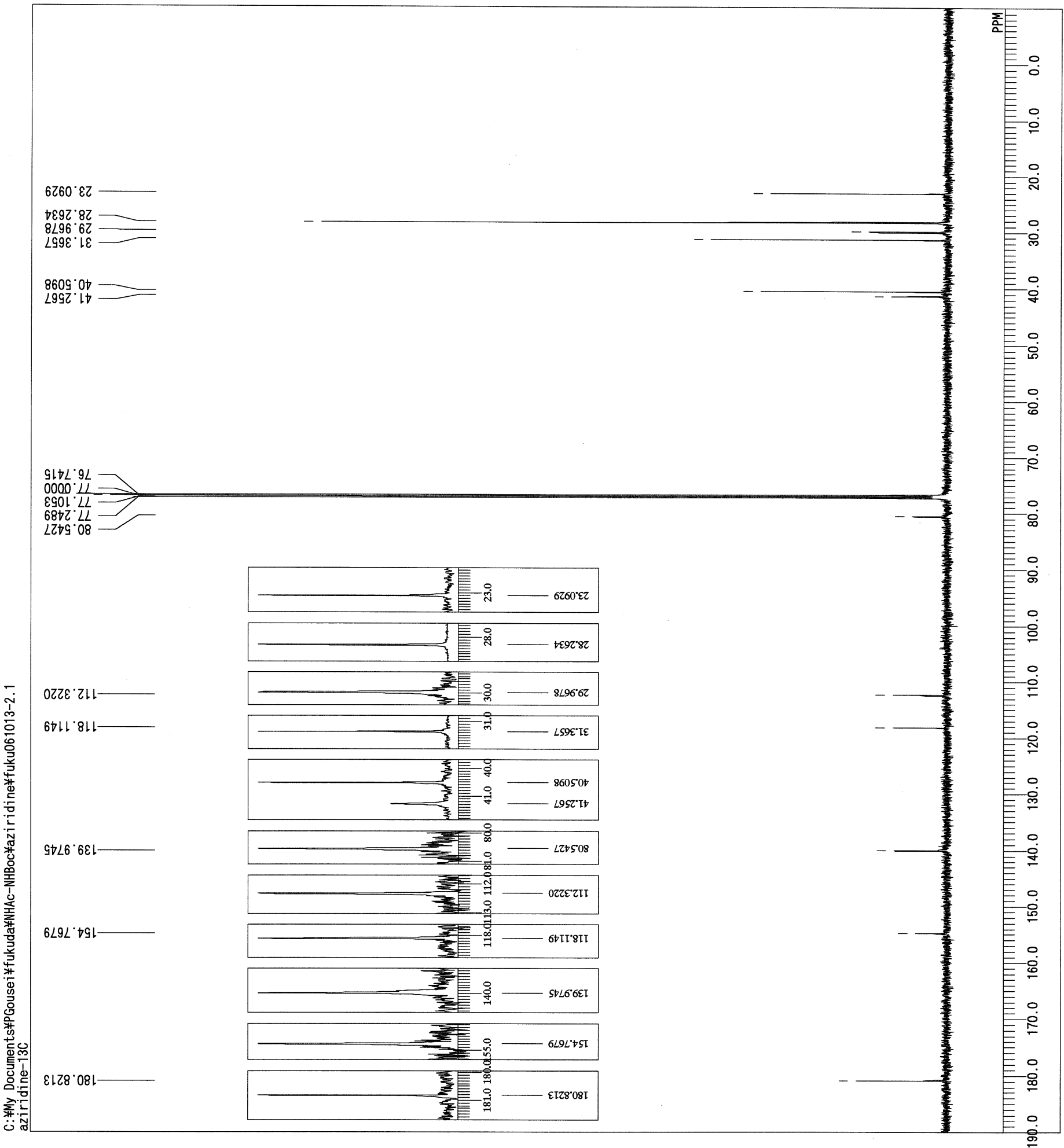




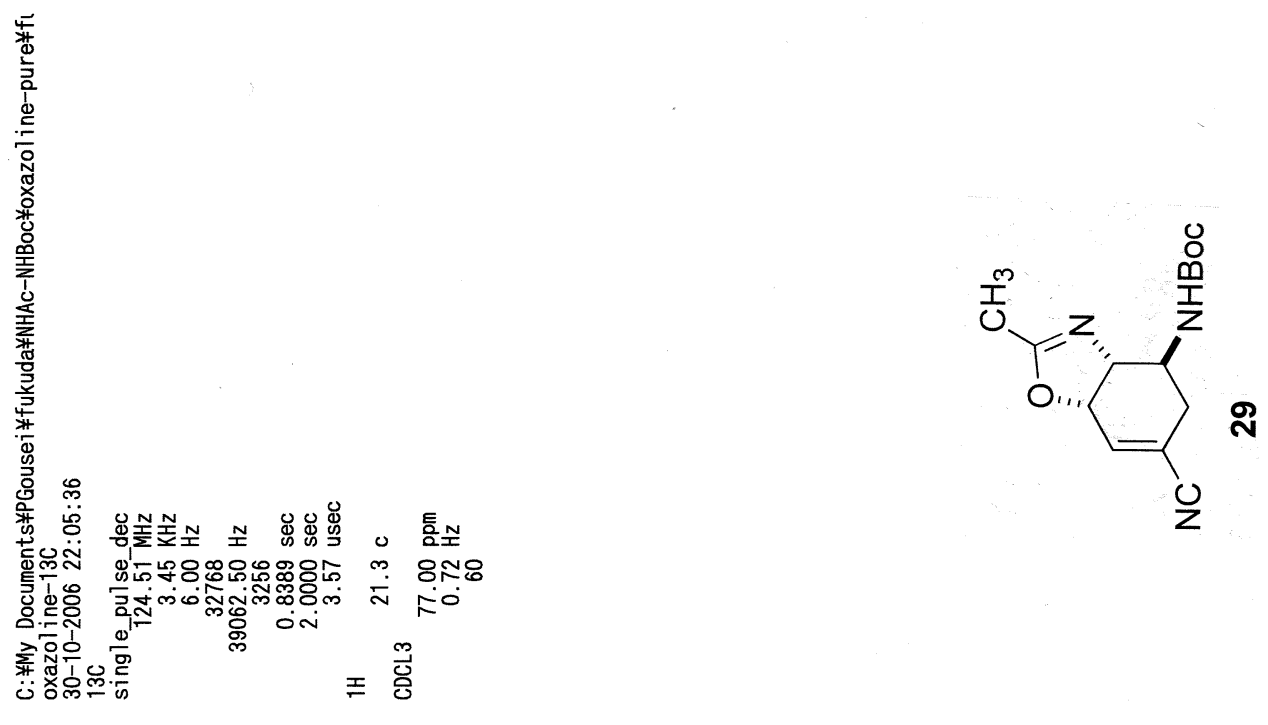

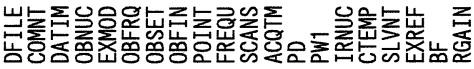

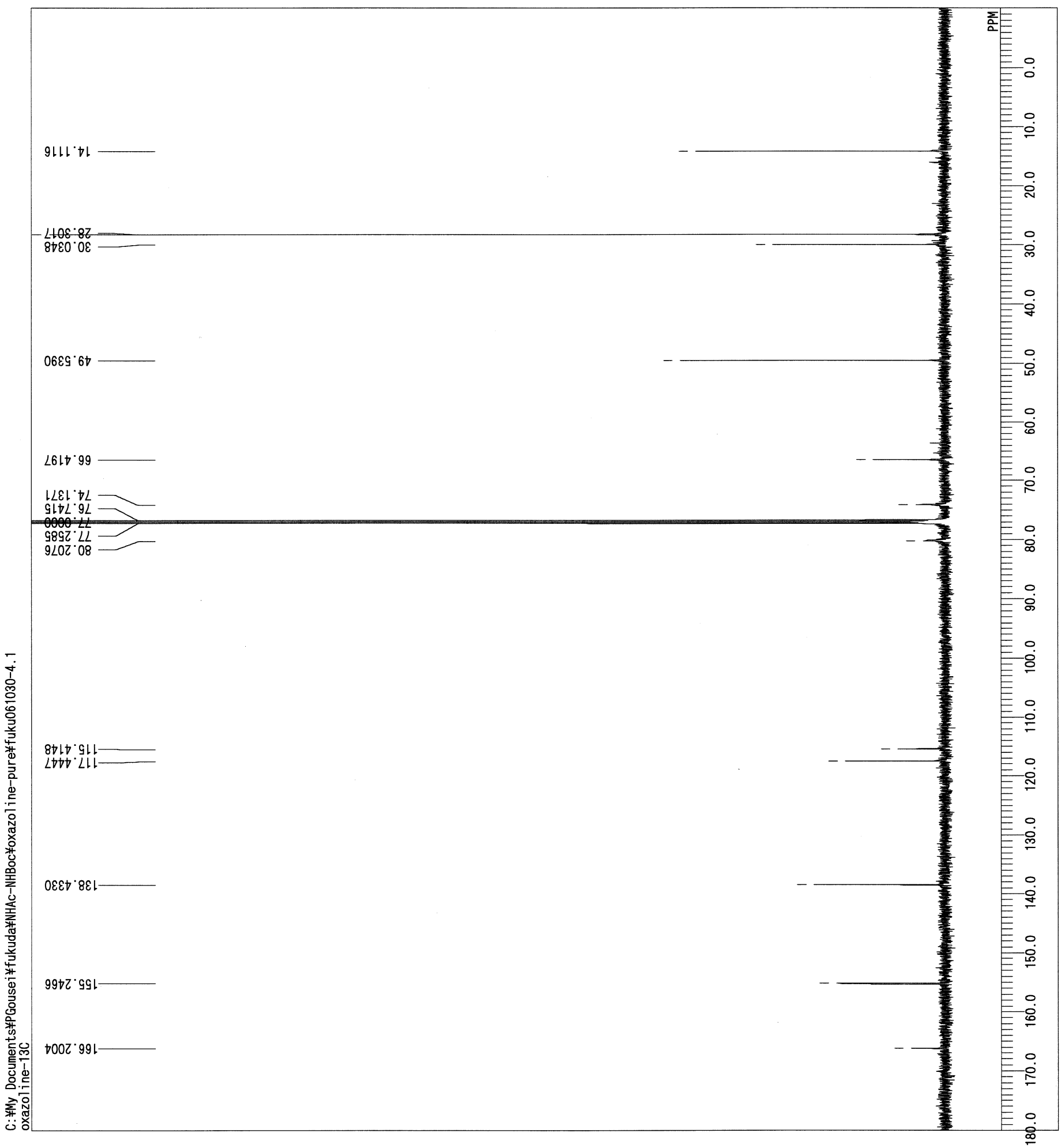

